Florida International University FIU Digital Commons

3-20-2013

\title{
The Effect of Iron Oxide Nanoparticles on the Fate and Transformation of Arsenic in Aquatic Environments
}

Dionne Dickson

Florida International University, ddick003@fiu.edu

DOI: $10.25148 /$ etd.FI13042321

Follow this and additional works at: https://digitalcommons.fiu.edu/etd

Part of the Analytical Chemistry Commons, and the Environmental Chemistry Commons

\section{Recommended Citation}

Dickson, Dionne, "The Effect of Iron Oxide Nanoparticles on the Fate and Transformation of Arsenic in Aquatic Environments" (2013). FIU Electronic Theses and Dissertations. 858.

https://digitalcommons.fiu.edu/etd/858 


\section{FLORIDA INTERNATIONAL UNIVERSITY}

Miami, Florida

THE EFFECT OF IRON OXIDE NANOPARTICLES ON THE FATE AND

TRANSFORMATION OF ARSENIC IN AQUATIC ENVIRONMENTS

A dissertation submitted in partial fulfillment of

the requirements for the degree of

DOCTOR OF PHILOSOPHY

in

CHEMISTRY

by

DIONNE C. DICKSON

2013 
To: Dean Kenneth Furton

College of Arts and Sciences

This dissertation, written by Dionne C. Dickson, and entitled The Effect of Iron Oxide Nanoparticles on the Fate and Transformation of Arsenic in Aquatic Environments, having been approved in respect to style and intellectual content, is referred to you for judgment.

We have read this dissertation and recommend that it be approved.

Chenzhong Li

Joong-Ho Moon

Kevin O’Shea

Kathleen Rein

Yong Cai, Major Professor

Date of Defense: March 20, 2013

The dissertation of Dionne C. Dickson is approved.

Dean Kenneth G Furton

College of Arts and Sciences

Dean Lakshmi N. Reddi

University Graduate School

Florida International University, 2013 
(C) Copyright 2013 by Dionne C. Dickson

All rights reserved. 


\section{DEDICATION}

I dedicate this dissertation to my parents, Havis and Dewett Dickson, and to the rest of my family (Howard, Corcel, Bianca and Dewett R). Without their unconditional love, patience, support and belief in me, the completion of this work would not have been possible. 


\section{ACKNOWLEDGMENTS}

I offer my sincerest gratitude to my major Professor, Dr. Yong Cai, for his support and guidance throughout my graduate studies. I am truly grateful for his encouragement, patience and suggestions. I am thankful for his mentorship as he challenged me to grow not only as an experimentalist but as an independent thinker. I am thankful for my committee members, Dr. Chenzhong Li, Dr. Joong-Ho Moon, Dr. Kevin O'Shea and Dr. Kathleen Rein for their helpful insights and valuable suggestions to my research. I would like to give a special thanks to Dr. Guangliang Liu for his suggestions and helpful discussions regarding my research. I would like to give thanks to Dr. Milagros Delgado, Mayra Exposito and Ms. Zaida Morales for their support and continual encouragement. Special thanks to my current and past lab mates Dr. Lucy Yehiayan, Dr. Sen Chen, Dr. Yuxiang Mao, Dr. Yanbin Li, Szabina Stice, Vadim Katsenovich, Ping Jiang, Yongmin Wang, Wenbin Cui and Mingwei Yang for their assistance, friendship and for some much needed humor in the lab making a stressful laboratory environment more pleasant. I would like to thank Dionne Blair and Diane Williams for the friendship and support. I would like to give special thanks to Ricardo Gil for his unwavering love, support, patience and encouragement throughout my entire journey in graduate school. I would like to express my sincerest gratitude to my beloved family for their faith in me, unconditional support and endless love they have given me my entire life. Finally, I would like to thank Florida International University Department of Chemistry and Biochemistry and the Graduate School for their financial support and Dissertation Year Fellowship. 


\begin{abstract}
OF THE DISSERTATION
THE EFFECT OF IRON OXIDE NANOPARTICLES ON THE FATE AND TRANSFORMATION OF ARSENIC IN AQUATIC ENVIRONMENTS
\end{abstract} by

Dionne C. Dickson

Florida International University, 2013

Miami, Florida

Professor Yong Cai, Major Professor

Iron oxides and arsenic are prevalent in the environment. With the increase interest in the use of iron oxide nanoparticles (IONPs) for contaminant remediation and the high toxicity of arsenic, it is crucial that we evaluate the interactions between IONPs and arsenic. The goal was to understand the environmental behavior of IONPs in regards to their particle size, aggregation and stability, and to determine how this behavior influences IONPs-arsenic interactions.

A variety of dispersion techniques were investigated to disperse bare commercial IONPs. Vortex was able to disperse commercial hematite nanoparticles into unstable dispersions with particles in the micrometer size range while probe ultrasonication dispersed the particles into stable dispersions of nanometer size ranges for a prolonged period of time. Using probe ultrasonication and vortex to prepare IONPs suspensions of different particle sizes, the adsorption of arsenite and arsenate to bare hematite nanoparticles and hematite aggregates were investigated. To understand the difference in the adsorptive behavior, adsorption kinetics and isotherm parameters were determined. Both arsenite and arsenate were capable of adsorbing to hematite nanoparticles and 
hematite aggregates but the rate and capacity of adsorption is dependent upon the hematite particle size, the stability of the dispersion and the type of sorbed arsenic species. Once arsenic was adsorbed onto the hematite surface, both iron and arsenic can undergo redox transformation both microbially and photochemically and these processes can be intertwined. Arsenic speciation studies in the presence of hematite particles were performed and the effect of light on the redox process was preliminary quantified. The redox behavior of arsenite and arsenate were different depending on the hematite particle size, the stability of the suspension and the presence of environmental factors such as microbes and light. The results from this study are important and have significant environmental implications as arsenic mobility and bioavailability can be affected by its adsorption to hematite particles and by its surface mediated redox transformation. Moreover, this study furthers our understanding on how the particle size influences the interactions between IONPs and arsenic thereby clarifying the role of IONPs in the biogeochemical cycling of arsenic. 


\section{TABLE OF CONTENTS}

CHAPTER

PAGE

I

Introduction 1

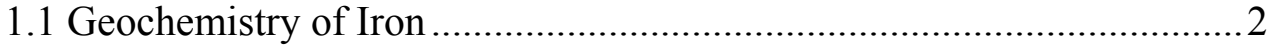

1.2 Nanotechnology and the Application of Iron Nanoparticles in Environmental Remediation .................................................................... 3

1.3 Arsenic in the Environment ............................................................. 7

1.4 Interactions between Arsenic and Iron Oxide Nanoparticles ...............15

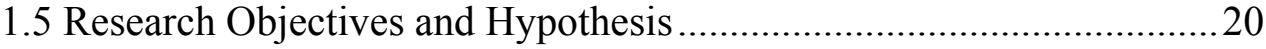

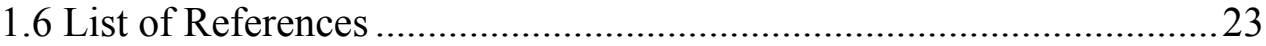

II Dispersion and stability of bare hematite nanoparticles: effect of dispersion tools, nanoparticle concentration, humic acid and ionic strength..............28

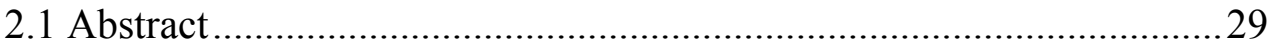

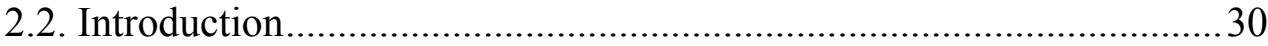

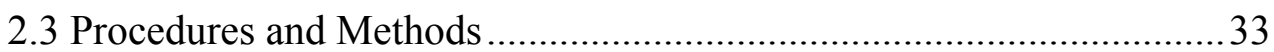

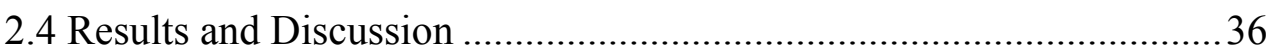

2.5 Conclusions................................................................................. 51

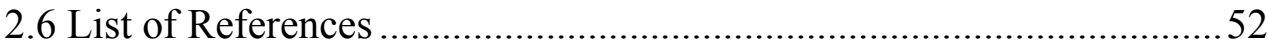

III Interactions of arsenite and arsenate with hematite nanoparticles and aggregates: sorption kinetics and isotherm studies...............................56

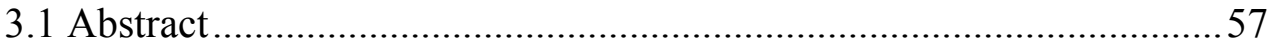

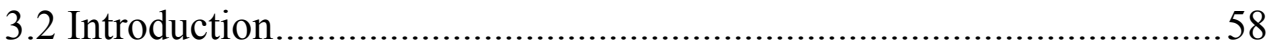

3.3 Procedures and Methods ...............................................................6 62

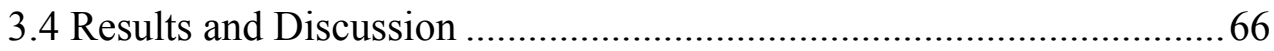

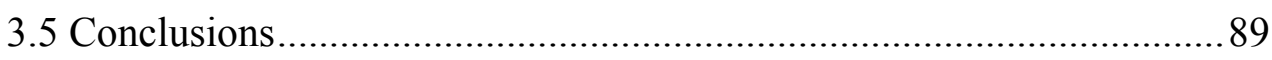

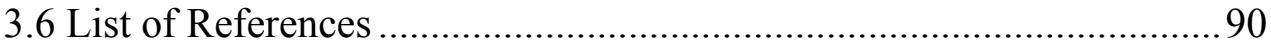

IV Redox transformation of arsenic in the presence of hematite nanoparticles and hematite aggregates............................................................. 94

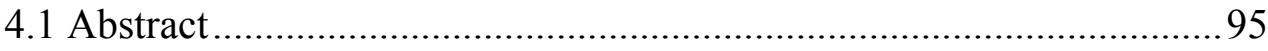

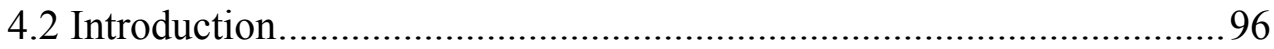

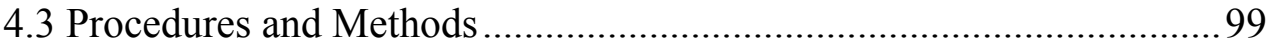

4.4 Results and Discussion ............................................................... 102

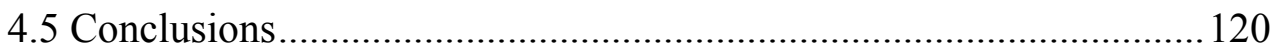

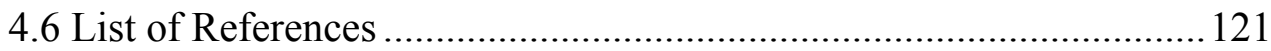

V Summary and Future Research Directions ....................................... 124

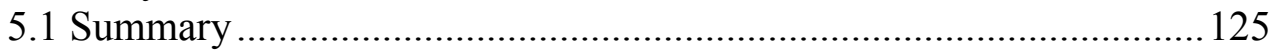

5.2 Future Research Directions.................................................... 128

VITA 


\section{LIST OF TABLES}

TABLE

PAGE

Table 1. 1 Major Iron Oxides Minerals in the Environment ................................ 3

Table 1.2 The most common arsenic minerals in the environment ....................... 8

Table 3. $1 \quad \mathrm{pH}$ measurements during As(III) and As(V) adsorption to hematite nanoparticles over a contact time of $168 \mathrm{~h}$.

Table 3. 2 Kinetic (pseudo second order) parameters for the adsorption of As(III) and $\mathrm{As}(\mathrm{V})$ with hematite nanoparticles and hematite aggregates....................................................................... 75

Table 3. 3 Freundlich isotherm parameters for the adsorption of As(III) and As(V) with hematite nanoparticles and hematite aggregates.......................... 85

Table 3. 4 Arsenic adsorption capacity to iron oxide minerals ............................ 88 


\section{LIST OF FIGURES}

FIGURE

PAGE

Figure 1.1 Structures of the most common arsenic species in the environment

Figure 1.2 Schematic representation of the possibly interactions of iron oxide nanoparticles with $\mathrm{As}(\mathrm{III})$ and $\mathrm{As}(\mathrm{V})$. (a) Adsorption and desorption of $\mathrm{As}(\mathrm{III})$ and As(V) to IONPs, (b) adsorption of As(III) and As(V) to IONPs followed by co-precipitation with IONPs, (c) reduction of As(V) to As(III) followed by desorption, and (d) oxidation of As(III) to As(V) followed by desorption.

Figure 2. 1 The time dependent hydrodynamic diameter of 10, 30, 50, 75, 100 and 200 $\mathrm{mg} / \mathrm{L}$ IONPs dispersed by (a) vortex for 20 minutes at power level 10 and (b) ultrasonic probe for 20 minutes at power level 6. 37

Figure 2. 2 The percentage of IONPs dispersed over a course of 168 hours for a 10 $\mathrm{mg} / \mathrm{L}$ IONPs dispersion at two power levels of sonication and at sonication times of 5, 20 and 60 minutes. Graphs a, b and c represent dispersion for 5, 20 and 60 minutes of probe ultrasonication at power level 3. Graphs d, e and f represent dispersion for 5, 20 and 60 minutes of probe ultrasonication, respectively at power level 6

Figure 2. 3 The percentage of IONPs dispersed over a course of 168 hours for a 100 $\mathrm{mg} / \mathrm{L}$ IONPs dispersed by ultrasonic probe. Graphs a, b and c represent dispersion for 5, 20 and 60 minutes respectively, at power level 3. Graphs d, e and f represent dispersion for 5, 20 and 60 minutes, respectively at power level 6 .

Figure 2. 4 The time dependent hydrodynamic diameter of 10, 30, 50, 75, 100 and 200 $\mathrm{mg} / \mathrm{L}$ IONPs dispersed by ultrasonic probe in the presence of humic acid $(100 \mathrm{mg} / \mathrm{L})$. All samples were dispersed using ultrasonic probe for 20 minutes at power level 6 .

Figure 2.5 The percentage of IONPs dispersed over a course of 168 hours while investigating the effect of $0.1 \mathrm{mM} \mathrm{NaCl}$ and $100 \mathrm{mg} / \mathrm{L} \mathrm{HA}$ (alone or in combination) on the dispersion of IONPs. Graphs a, b, c, d, e and $\mathrm{f}$ represent the percentage of IONPs dispersed in 10, 30, 50, 75, 100 and $200 \mathrm{mg} / \mathrm{L}$ IONPs suspension respectively. All samples were dispersed using ultrasonic probe for 20 minutes at power level 6 .

Figure 2. 6 The time dependent hydrodynamic diameter of 10, 30, 50, 75, 100 and 200 $\mathrm{mg} / \mathrm{L}$ IONPs dispersed by ultrasonic probe in the presence of $\mathrm{NaCl}(0.1$ 
$\mathrm{mM}$ ). All samples were dispersed using ultrasonic probe for 20 minutes at power level 6 .

Figure 2.7 The time dependent hydrodynamic diameter of 100 and $200 \mathrm{mg} / \mathrm{L}$ IONPs dispersions in the presence of $10 \mathrm{mM} \mathrm{NaCl}$. Samples were dispersed using ultrasonic probe for 20 minutes at power level 6 .

Figure 2.8 The time dependent hydrodynamic diameter of 10, 30, 50, 75, 100 and 200 $\mathrm{mg} / \mathrm{L}$ IONPs dispersed by ultrasonic probe in the presence of HA (100 $\mathrm{mg} / \mathrm{L})$ and $\mathrm{NaCl}(0.1 \mathrm{mM})$. All samples were dispersed using ultrasonic probe for 20 minutes at power level 6 .

Figure 3. 1 SEM micrographs of (a) hematite nanoparticles $(10 \mathrm{mg} / \mathrm{L})$ in water dispersed using probe ultrasonication (b) hematite aggregates $(10 \mathrm{mg} / \mathrm{L})$ dispersed using vortex

Figure 3.2 The effect of centrifugation on the separation of IONPs from aqueous phase. A $10 \mathrm{mg} / \mathrm{L}$ IONPs dispersion was centrifuged at 10,000 and 6,000 rpm for 30 and 60 minutes

Figure 3. 3 The effect of filtration on the separation of IONPs from aqueous phase. A $10 \mathrm{mg} / \mathrm{L}$ IONPs dispersion was filtered using $0.45 \mu \mathrm{m}$ PVDF, $0.22 \mu \mathrm{m}$ PVDF and $0.22 \mu \mathrm{m}$ nylon filter.

Figure 3. 4 Plots of arsenic adsorbed onto hematite nanoparticle. a) As(III) adsorbed onto hematite nanoparticles ( $\mu \mathrm{g}$ As(III)/g IONPs) b) As(V) adsorbed onto hematite nanoparticles ( $\mu \mathrm{g}$ As(V)/g IONPs). Adsorption was performed using $200 \mu \mathrm{g} / \mathrm{L} \mathrm{As}(\mathrm{III})$ or As(V) and $10 \mathrm{mg} / \mathrm{L}$ hematite nanoparticles over a contact time of $168 \mathrm{~h}$ .71

Figure 3.5 Linear Pseudo-Second Order Kinetic Fit for the adsorption of (a) As(III) to hematite nanoparticles and (b) As(V) to hematite nanoparticles. .74

Figure 3.6 Hematite nanoparticle concentration (squares) and particle size distribution (triangles) monitored during adsorption (contact time of 168h) (a) As(III) and (b) As(V).

Figure 3.7 Plots of arsenic adsorbed onto hematite aggregates. a) As(III) adsorbed onto hematite aggregates ( $\mu$ g As(III)/g IONPs) b) As(V) adsorbed onto hematite aggregates. Adsorption was performed using $200 \mu \mathrm{g} / \mathrm{L} \mathrm{As(III)}$ and $\mathrm{As}(\mathrm{V})$ and $10 \mathrm{mg} / \mathrm{L}$ hematite aggregates over a contact time of $168 \mathrm{~h}$.

Figure 3.8 Linear Pseudo-Second Order Kinetic Fit for the adsorption of (a) As(III) to hematite aggregates and (b) of $\mathrm{As}(\mathrm{V})$ to hematite aggregates. 
Figure 3.9 Freundlich Isotherm of (a) As(III) and (b) As(V) with hematite nanoparticles. Arsenic concentrations vary from $10-2000 \mu \mathrm{g} / \mathrm{L}$ and reacted with $10 \mathrm{mg} / \mathrm{L}$ of hematite nanoparticles to equilibrium (24h). .83

Figure 3.10 Freundlich Isotherm of (a) As(III) and (b) As(V) with hematite aggregate. Arsenic concentrations vary from $10-2000 \mu \mathrm{g} / \mathrm{L}$ and reacted with $10 \mathrm{mg} / \mathrm{L}$ of hematite aggregates to equilibrium (24h). .86

Figure 4. 1 Arsenite speciation studies over a contact time of 168h. (a) As(III) (200 $\mu \mathrm{g} / \mathrm{L}$ ) with $10 \mathrm{mg} / \mathrm{L}$ hematite nanoparticles under room light at $20^{\circ} \mathrm{C}$ and (b) $\mathrm{As}(\mathrm{III})$ control $(200 \mu \mathrm{g} / \mathrm{L}$ of $\mathrm{As}(\mathrm{III})$ in deionized water under room light at $\left.20^{\circ} \mathrm{C}\right)$ 103

Figure 4. 2 Arsenate speciation studies over a contact time of 168h. (a) As(V) (200 $\mu \mathrm{g} / \mathrm{L}$ ) with $10 \mathrm{mg} / \mathrm{L}$ hematite nanoparticles under room light at $20^{\circ} \mathrm{C}$ and (b) $\mathrm{As}(\mathrm{V})$ control 104

Figure 4.3 Arsenite speciation studies over a contact time of 168h. (a) As(III) (200 $\mu \mathrm{g} / \mathrm{L}$ ) with $10 \mathrm{mg} / \mathrm{L}$ hematite aggregates under room light at $20^{\circ} \mathrm{C}$ and (b) As(III) control 106

Figure 4. 4 Arsenate speciation studies over a contact time of 168h. (a) As(V) (200 $\mu \mathrm{g} / \mathrm{L}$ ) with $10 \mathrm{mg} / \mathrm{L}$ hematite aggregates under room light at $20^{\circ} \mathrm{C}$ and (b) As $(\mathrm{V})$ control

Figure $4.5 \quad \operatorname{As}(\mathrm{III})(250 \mu \mathrm{g} / \mathrm{L})$ speciation studies in the presence of hematite nanoparticles $(10 \mathrm{mg} / \mathrm{L})$ in (a) light (b) dark. The columns represent arsenic in solution $(\mu \mathrm{g} / \mathrm{L})$. The line represents the amount of arsenic is adsorbed $(\mu \mathrm{g} / \mathrm{L})$ onto the hematite nanoparticles. 109

Figure 4. $6 \quad$ As(III) $(200 \mu \mathrm{g} / \mathrm{L})$ speciation studies in the presence of hematite aggregates $(10 \mathrm{mg} / \mathrm{L})$ in (a) light (b) dark. The columns represent arsenic in solution $(\mu \mathrm{g} / \mathrm{L})$ and the line represents the amount of arsenic $(\mu \mathrm{g} / \mathrm{L})$ was adsorbed onto the hematite aggregates. 111

Figure 4. 7 As(III) control $(250 \mu \mathrm{g} / \mathrm{L})$ performed during the As(III)-hematite nanoparticles speciation experiment (a) light (b) dark

Figure 4. 8 As(III) control $(200 \mu \mathrm{g} / \mathrm{L})$ performed during the As(III)-hematite aggregates speciation experiment (a) light (b) dark.

Figure 4. 9 Proposed pathway for surface mediated As(III) oxidation in the presence of $\alpha-\mathrm{Fe}_{2} \mathrm{O}_{3}$ (hematite). After light absorption, a strong reducing electron and a strong oxidizing hole are formed. Fe(III) is reduced to Fe(II) by the 
reducing electron and $\mathrm{As}(\mathrm{III})$ is oxidized to $\mathrm{As}(\mathrm{V})$ via the oxidizing hole, $\mathrm{h}^{+}$. Both Fe(II) and As(V) are then released into solution.

Figure 4.10 As(V) $(250 \mu \mathrm{g} / \mathrm{L})$ speciation studies in the presence of hematite nanoparticles in (a) light (b) dark. The columns represent arsenic in solution $(\mu \mathrm{g} / \mathrm{L})$ 116

Figure 4. 11 As(V) $(200 \mu \mathrm{g} / \mathrm{L})$ speciation studies in the presence of hematite aggregates in (a) light (b) dark. The columns represent arsenic in solution $(\mu \mathrm{g} / \mathrm{L}) .116$

Figure 4. $12 \mathrm{As}(\mathrm{V})$ control $(250 \mu \mathrm{g} / \mathrm{L})$ performed during the As(V)-hematite nanoparticles speciation experiment (a) light (b) dark.

Figure 4. 13 As(V) control $(200 \mu \mathrm{g} / \mathrm{L})$ performed during the As(V)-hematite aggregates speciation experiment (a) light (b) dark.

Figure 4. 14 Sedimentation of hematite nanoparticles $(10 \mathrm{mg} / \mathrm{L})$ in the presence of $0.02 \%$ sodium azide over a period of $144 \mathrm{~h}$. 


\section{LIST OF ABBREVIATIONS AND ACRONYMS}

ACRONYM

As

As(III)

$\operatorname{As}(\mathrm{V})$

AsBet

AsCho

ATSDR

DLS

DLVO

DMA

DOM

$\mathrm{E}_{\mathrm{h}}$

EPA

$\mathrm{Fe}$

FE-SEM

GFAAS

$\mathrm{h}$

HA

HPLC-ICP/MS

IARC

ICP-MS

IONPs
FULL NAME

Arsenic

Arsenite

Arsenate

Arsenobetaine

Arsenocholine

Agency for toxic substances and disease registry

Dynamic Light Scattering

Derjaguin-Landau-Verwey- Overbeek

Dimethylarsinic acid

Dissolved organic matter

Redox potential

Environmental Protection Agency

Iron

Field emission scanning electron microscopy

Graphite furnace atomic absorption spectrometry

hour

Humic Acid

High performance liquid chromatography

International Agency for Research on Cancer

Inductively coupled plasma spectrometry

Iron oxide nanoparticles 


$\begin{array}{ll}\text { MMA } & \text { Monomethylarsonic acid } \\ \mathrm{NaCl} & \text { Sodium Chloride } \\ \mathrm{nm} & \text { Nanometer } \\ \mathrm{NOM} & \text { Natural organic matter } \\ \text { NZVI } & \text { Nanoscale zerovalent iron } \\ \text { O } & \text { Oxygen } \\ \text { OH } & \text { Hydroxyl } \\ \text { PAA } & \text { Polyacrylic acid } \\ \text { PCB } & \text { Polychlorinated biphenyls } \\ \text { PZC } & \text { Point zero charge } \\ \text { S } & \text { Sulfur } \\ \text { SEM } & \text { Scanning electron microscope } \\ \text { TCE } & \text { Tricholoethylene } \\ \text { TMAs } & \text { Tetramethylarsonium ion } \\ \text { TMAO } & \text { Trimethylarsine oxide } \\ \text { USA } & \text { United States of America } \\ \text { WHO } & \text { World health organization }\end{array}$




\section{CHAPTER I}

Introduction 


\subsection{Geochemistry of Iron}

Iron is widespread in nature and is the fourth most abundant element in the earth's crust (Schwertmann et al., 2000; Taylor et al., 2011). Elemental iron is highly reactive and thus rarely exists as zerovalent iron, $\mathrm{Fe}(0)$, in the environment. Because of the high reactivity, iron has variable oxidation states which allow it to coordinate with oxygen, sulfur and nitrogen atoms. The two major oxidation states of iron in the environment is the reduced water-soluble $\mathrm{Fe}(\mathrm{II})$ (ferrous iron) and the oxidized water-insoluble Fe(III) (ferric iron) (Bose et al., 2002; Cundy et al., 2008). These oxidation states allow iron to exist in nature as either dissolved ions or in different solid mineral forms. For example, $\mathrm{Fe}(0)$ can readily react with oxygen in air or water to from iron oxides, oxide hydroxides and hydroxides minerals (Cundy et al., 2008). In addition, iron also exist in other mineral forms such as carbonates, phosphates, sulfates, sulfides and silicates (Jambor et al., 1998).

Iron oxides minerals are the most widespread in the environment and are ubiquitous in air, soils, rocks, lakes and rivers, and on the sea floor (Schwertmann et al., 2000). The term "iron oxides" is used for simplicity as iron oxides come in different chemical and structural forms such as iron oxides, oxide hydroxides and hydroxides. So far, there are fifteen different iron oxides consisting only of $\mathrm{Fe}, \mathrm{O}$ and/or $\mathrm{OH}$, however, they differ in the valence of Fe and crystal structure (Schwertmann et al., 2000). Hematite, goethite, lepidocrocite and ferihydrite are some of the major iron oxides minerals in the environment (Table 1-1). Each mineral form has different stability, reactivity and specific surface area (Jambor et al., 1998). Despite their chemical and structural forms, iron oxides play a significant role in many biogeochemical processes in the environment. 
Table 1. 1 Major Iron Oxides Minerals in the Environment (Schwertmann et al., 2000)

\begin{tabular}{|c|c|c|c|}
\hline Iron oxides & Mineral & Formula & Environmental occurrence \\
\hline \multirow[t]{4}{*}{ Oxides } & Hematite & $\alpha-\mathrm{Fe}_{2} \mathrm{O}_{3}$ & $\begin{array}{l}\text { Widespread in rocks, soils } \\
\text { and water }\end{array}$ \\
\hline & Magnetite & $\mathrm{Fe}_{3} \mathrm{O}_{4}$ & $\begin{array}{l}\text { Igneous and metamorphic } \\
\text { rocks }\end{array}$ \\
\hline & Ferrihydrite & $\mathrm{Fe}_{5} \mathrm{HO}_{8} .4 \mathrm{H}_{2} \mathrm{O}$ & $\begin{array}{l}\text { Fe containing springs, } \\
\text { drainage lines, lake oxide } \\
\text { precipitates, groundwater, } \\
\text { river sediments, oceans }\end{array}$ \\
\hline & Maghemite & $\gamma-\mathrm{Fe}_{2} \mathrm{O}_{3}$ & $\begin{array}{l}\text { Soils as the weathering } \\
\text { product of magnetite }\end{array}$ \\
\hline \multirow[t]{4}{*}{ Oxyhydroxides } & Goethite & $\alpha-\mathrm{FeOOH}$ & Soils, lakes, streams \\
\hline & Akaganeite & $\beta-\mathrm{FeOOH}$ & $\begin{array}{l}\text { Chloride-rich } \\
\text { environments such as hot } \\
\text { brines and in rust in marine } \\
\text { environments }\end{array}$ \\
\hline & Lepidocrocite & $\gamma$-FeOOH & Rocks, soils, biota and rust \\
\hline & Feroxyhyte & $\delta^{\prime}-\mathrm{FeOOH}$ & Soils \\
\hline
\end{tabular}

\subsection{Nanotechnology and the Application of Iron Nanoparticles in Environmental}

\section{Remediation}

\subsubsection{Nanotechnology and Environmental Remediation}

Organic and heavy metal contamination of ground and surface waters has a major impact on the environment, the economy and on human health. Groundwater and surface water pollution is a significant problem throughout the world and the need for potable water in developing and developed countries is necessary for the health and survival of 
humans and animals. The poor water quality in some industrial and agricultural sites caused a reduction in the supply of freshwater for human use (Theron et al., 2008). The need for clean water and the financial burden on cleaning up contaminated sites can be challenging for countries and regions that are not economically stable. The need for a new and economically feasible technology to address these pollution issues led to the potential application of nanotechnology for remediation purposes.

Nanotechnology offers the potential use of novel nanomaterials for treatment of surface water, groundwater, and wastewater contaminated by toxic metal ions, organic and inorganic solutes, and microorganisms (Sun et al., 2006; Theron et al., 2008). Nanomaterials are generally defined as particles with dimensions ranging from 1 to 100 $\mathrm{nm}$. The small particle size and large surface area to volume ratio account for their high reactivity (large number of reactive sites) and easy delivery through small spaces in the subsurface to contaminated sites (U.S. EPA, 2008). These nanomaterials are highly preferred for in-situ remediation because of time and cost effectiveness. In-situ remediation involves treatment occurring on site thereby eliminating the need to pump out groundwater for above ground treatment or the transportation of soil and water to any other places for contaminant clean up (U.S. EPA, 2000; Karn et al., 2009). These advantages sparked a great interest in nanoparticles for environmental applications. In the last decade, the application of nanoscale zerovalent iron (NZVI) has emerged as the leading nanomaterial for environmental remediation. 


\subsubsection{Nanoscale Zerovalent Iron (NZVI) and Iron Oxide Nanoparticles (IONPs) Remediation Technology}

Nanoscale zerovalent iron can be considered as one of the first generation nanoscale environmental technologies (Sun et al., 2006) and its environmental application has significantly increased over the last decade. Nanoscale zerovalent iron, a cost effective remediation tool, has shown potential in cleaning up difficult remediation sites (Zhang, 2003). The greatest applications for NZVI is the in-situ groundwater remediation of chlorinated organic compounds such as trichloroethylene (TCE), organochlorine pesticides, polychlorinated biphenyls (PCBs) and various toxic metals such as arsenic and mercury (Wang et al., 1997; Liu et al., 2005; Quinn et al., 2005; Liu et al., 2006; Turk et al., 2010). The small particle size, large surface area, and high in-situ reactivity, accounts for its effectiveness as a reagent for the treatment of contaminants in soil, sediment, and groundwater remediation (Kanel et al., 2005). NZVI has a unique core-shell structure that influences its chemical properties. The core is composed of zerovalent iron $(\mathrm{Fe}(0))$ which is surrounded by an iron oxide/hydroxide shell that grows thicker with the progress of iron oxidation (Martin et al., 2008). Because of the high reactivity of elemental iron, the oxidation of the iron core is inevitable once NZVI is exposed to air or water during environmental applications (equations 1 and 2). Nanoscale zerovalent iron undergoes in situ oxidation (corrosion) to produce ferrous iron (Ponder et al., 2000).

$$
\begin{aligned}
& \mathrm{Fe}^{0}+2 \mathrm{H}_{2} \mathrm{O} \rightarrow \mathrm{Fe}^{2+}+\mathrm{H}_{2}+2 \mathrm{OH}^{-} \\
& \mathrm{Fe}^{0}+\mathrm{O}_{2}+2 \mathrm{H}_{2} \mathrm{O} \rightarrow 2 \mathrm{Fe}^{2+}+4 \mathrm{OH}^{-}
\end{aligned}
$$


Further reactions of $\mathrm{Fe}^{2+}$ (equations 3-6) will subsequently yield magnetite $\left(\mathrm{Fe}_{3} \mathrm{O}_{4}\right)$, ferrous hydroxide $\left[\mathrm{Fe}(\mathrm{OH})_{2}\right]$, and ferric hydroxide $\left[\mathrm{Fe}(\mathrm{OH})_{3}\right]$ depending upon redox conditions and $\mathrm{pH}$ (Kanel et al., 2005).

$$
\begin{aligned}
& 6 \mathrm{Fe}^{2+}+\mathrm{O}_{2}+6 \mathrm{H}_{2} \mathrm{O} \rightarrow 2 \mathrm{Fe}_{3} \mathrm{O}_{4}+12 \mathrm{H}^{+} \\
& \mathrm{Fe}^{2+}+2 \mathrm{OH}^{-} \rightarrow 2 \mathrm{Fe}(\mathrm{OH})_{2} \\
& 6 \mathrm{Fe}(\mathrm{OH})_{2}+\mathrm{O}_{2} \rightarrow 2 \mathrm{Fe}_{3} \mathrm{O}_{4}+6 \mathrm{H}_{2} \mathrm{O} \\
& \mathrm{Fe}_{3} \mathrm{O}_{4}+\mathrm{O}_{2}+18 \mathrm{H}_{2} \mathrm{O} \leftrightarrow 12 \mathrm{Fe}(\mathrm{OH})_{3}
\end{aligned}
$$

Most importantly, the oxide layer is composed of $\mathrm{Fe}(\mathrm{II}) / \mathrm{Fe}(\mathrm{III})$ oxides near the $\mathrm{Fe}(0)$ surface with the Fe(III) oxide being closer to the oxide/water interface (Yan et al., 2010). The unique core-shell structure provides an indication of the chemical properties and reactivity of NZVI. The engineered iron nanoparticles may exhibit characteristics of both metallic iron and iron oxides. The metallic iron may act as a strong reductant (electron donor), while the oxide layer may act as a sorbent for contaminants through electrostatic interactions or surface complexation processes (Sun et al., 2006; Yan et al., 2010).

The NZVI oxide layer is very important in contaminant remediation. The oxide layer can be composed of any of the major iron oxides minerals. Because of this, various iron oxide nanoparticles (IONPs) such as goethite, magnetite and hematite have been investigated for potential applications in environmental remediation (Rebodos et al., 2010; Shipley et al., 2010). Similar to NZVI, IONPs have small particle size, large surface area, and high reactivity. These IONPs also have similar adsorptive behavior as the NZVI oxide layer and are frequently used as a model system for understanding the behavior of NZVI in the environment (He et al., 2008). As a sorbent, IONPs can adsorb metal 
contaminants onto their surface during remediation and facilitate their mobility and transport in the environment.

\subsection{Arsenic in the Environment}

\subsubsection{Occurrence and Speciation}

Arsenic (As) is the $20^{\text {th }}$ most abundant element in natural systems and is extremely toxic to humans and animals (Carabante et al., 2009). It is naturally present in the earth's crust, soils, sediments, water, air and living organism (Mandal et al., 2002; Zhang et al., 2002). Arsenic occurs naturally in more than 245 mineral forms but it is most often associated with sulfur (S) containing minerals (Bissen et al., 2003). Table 1-2 lists the most commonly found arsenic minerals and their occurrence in the environment. Arsenopyrite (FeAsS) and orpiment $\left(\mathrm{As}_{2} \mathrm{~S}_{3}\right)$ are the most common arsenic minerals in nature and when exposed to oxidizing water they will undergo oxidative dissolution to release Fe, As and S into the environment (Yunmei et al., 2004; Suess et al., 2012). Arsenic in soils vary amongst geological regions but the global average concentration is approximately $5 \mathrm{mg} \mathrm{kg}^{-1}$ (Chakraborti, 2011). In sediments, arsenic concentrations will vary $\left(0.1-4000 \mathrm{mg} \mathrm{kg}^{-1}\right)$ depending on where the sediments are originated such as from lakes, rivers and streams (Chakraborti, 2011). Arsenic concentrations of $1-2 \mu \mathrm{g} \mathrm{L}^{-1}$ can be found in seawater while $10 \mu \mathrm{g} \mathrm{L}^{-1}$ or less can be found in unpolluted ground and surface water (Chakraborti, 2011). In air, arsenic concentrations ranged from 0.02 to $4 \mathrm{ng} \mathrm{m}^{-3}$ in rural areas, 3-200 $\mathrm{ng} \mathrm{m}^{-3}$ in urban areas to $>1000 \mathrm{ng} \mathrm{m}^{-3}$ in industrial areas (WHO, 2011). 
Table 1. 2 The most common arsenic minerals in the environment (Mandal et al., 2002)

\begin{tabular}{|c|c|c|}
\hline Mineral & Formula & Occurrence in the environment \\
\hline Native As & As & Hydrothermal veins \\
\hline Arsenopyrite & FeAsS & $\begin{array}{l}\text { The most abundant As mineral, dominantly } \\
\text { mineral veins }\end{array}$ \\
\hline Proustite & $\mathrm{Ag}_{3} \mathrm{AsS}_{3}$ & $\begin{array}{l}\text { Generally one of the late } \mathrm{Ag} \text { minerals in } \\
\text { the sequence of primary deposition }\end{array}$ \\
\hline Rammelsbergite & $\mathrm{NiAs}_{2}$ & Commonly in mesothermal vein deposits \\
\hline Safflorite & $(\mathrm{Co}, \mathrm{Fe}) \mathrm{As}_{2}$ & Generally in mesothermal vein deposits \\
\hline Seligmannite & $\mathrm{PbCuAsS}_{3}$ & Occurs in hydrothermal veins \\
\hline Smaltite & $\mathrm{CoAs}_{2}$ & - \\
\hline Niccolite & NiAs & Vein deposits and norites \\
\hline Realgar & $\mathrm{AsS}$ & $\begin{array}{l}\text { Vein deposits, often associated with } \\
\text { orpiment, clays and limestones, also } \\
\text { deposits from hot springs }\end{array}$ \\
\hline Orpiment & $\mathrm{As}_{2} \mathrm{~S}_{3}$ & $\begin{array}{l}\text { Hydrothermal veins, hot springs, volcanic } \\
\text { sublimation product }\end{array}$ \\
\hline Cobaltite & CoAsS & $\begin{array}{l}\text { High-temperature deposits, metamorphic } \\
\text { rocks }\end{array}$ \\
\hline Tennantite & $(\mathrm{Cu}, \mathrm{Fe}) 12 \mathrm{As}_{4} \mathrm{~S}_{13}$ & Hydrothermal veins \\
\hline Enargite & $\mathrm{Cu}_{3} \mathrm{AsS}_{4}$ & Hydrothermal veins \\
\hline Arsenolite & $\mathrm{As}_{2} \mathrm{O}_{3}$ & $\begin{array}{l}\text { Secondary mineral formed by oxidation of } \\
\text { arsenopyrite, native arsenic and other } \\
\text { As minerals }\end{array}$ \\
\hline Claudetite & $\mathrm{As}_{2} \mathrm{O}_{3}$ & $\begin{array}{l}\text { Secondary mineral formed by oxidation of } \\
\text { realgar, arsenopyrite and other As } \\
\text { minerals }\end{array}$ \\
\hline Scorodite & $\mathrm{FeAsO}_{4} \cdot 2 \mathrm{H}_{2} \mathrm{O}$ & Secondary mineral \\
\hline Annabergite & $(\mathrm{Ni}, \mathrm{Co})_{3}\left(\mathrm{AsO}_{4}\right)_{2} \cdot 8 \mathrm{H}_{2} \mathrm{O}$ & Secondary mineral \\
\hline Hoernesite & $\mathrm{Mg}_{3}\left(\mathrm{AsO}_{4}\right)_{2} \cdot 8 \mathrm{H}_{2} \mathrm{O}$ & Secondary mineral, smelter wastes \\
\hline Haematolite & $\begin{array}{l}(\mathrm{Mn}, \mathrm{Mg})_{4} \mathrm{Al}\left(\mathrm{AsO}_{4}\right) \\
(\mathrm{OH})_{8}\end{array}$ & - \\
\hline Conichalcite & $\mathrm{CaCu}\left(\mathrm{AsO}_{4}\right)(\mathrm{OH})$ & Secondary mineral \\
\hline Adamite & $\mathrm{Zn}_{2}(\mathrm{OH})\left(\mathrm{AsO}_{4}\right)$ & Secondary mineral \\
\hline Domeykite & $\mathrm{Cu}_{3} \mathrm{As}$ & $\begin{array}{l}\text { Found in vein and replacement deposits } \\
\text { formed at moderate temperatures }\end{array}$ \\
\hline Loellingite & $\mathrm{FeAs}_{2}$ & Found in mesothermal vein deposits \\
\hline Pharmacosiderite & $\mathrm{Fe}_{3}\left(\mathrm{AsO}_{4}\right)_{2}(\mathrm{OH})_{3} \cdot 5 \mathrm{H}_{2} \mathrm{O}$ & $\begin{array}{l}\text { Oxidation product of arsenopyrite and } \\
\text { other As minerals }\end{array}$ \\
\hline
\end{tabular}


In living organisms, As concentrations will vary depending on how much arsenic they are exposed to. Animals and humans tend to have concentrations less than $0.3 \mathrm{ug} \mathrm{g}^{-1}$ on a wet weight basis (Mandal et al., 2002).

Arsenic is commonly introduced into the environment through both natural formation (weathering, biological activity, and volcanic activity) and anthropogenic activity (mining, smelting, and direct use of arsenic-containing herbicides by industry and agriculture) (Mandal et al., 2002). However, anthropogenic activities accounts for a higher release of arsenic, as much as three times higher, compared to natural sources (Mandal et al., 2002). In the environment, arsenic exists in various oxidation states $(+5$, $+3,0,-3)$ and two different chemical forms (inorganic or organic arsenicals). Figure 1.1 illustrates the most common arsenic form/species in the environment. In ground and surface waters the major chemical forms of arsenic are the inorganic arsenic species: pentavalent arsenate $(\mathrm{As}(\mathrm{V}))$ and trivalent arsenite $(\mathrm{As}(\mathrm{III}))$ (Bissen et al., 2003). However, these inorganic arsenics can interact with sulfur to form arsenic-sulfur species (thioarsenic). These thiolated species such as thiolated arsenites and thiolated arsenates can exist in Fe rich or sulfide rich waters especially where orpiment and arsenopyrite minerals undergo dissolution (Yunmei et al., 2004; Suess et al., 2012). Organic arsenicals are less prevalent in the environment but can be found in surface waters and in areas affected by industrial pollution. The most common forms are monomethylarsonic acid (MMA) and dimethylarsinic acid (DMA). MMA and DMA are usually present in plants, humans and animals resulting from the uptake and intake of inorganic arsenic from the environment. Arsenic is metabolized by methylation in the liver and is usually excreted in the urine (Rossman, 2003). Other known forms of organic arsenicals are trimethylarsine 
oxide (TMAO), the tetramethylarsonium ion (TMAs), arsenobetaine (AsBet), arsenocholine (AsCho) and dimethylarsinoylriboside derivatives (arsenosugars) (Kohlmeyer et al., 2003).

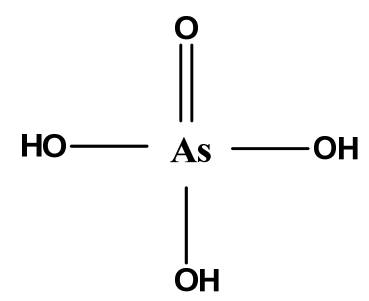

$\operatorname{As}(\mathbf{V})$

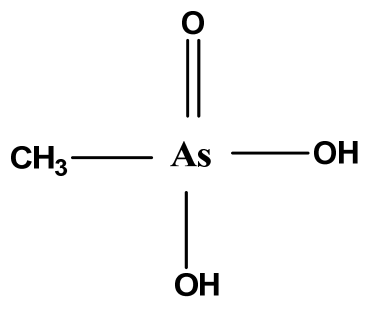

$\operatorname{MMA}(\mathbf{V})$

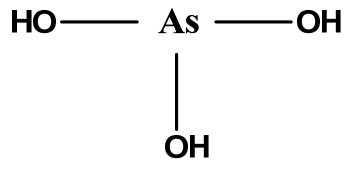

As(III)

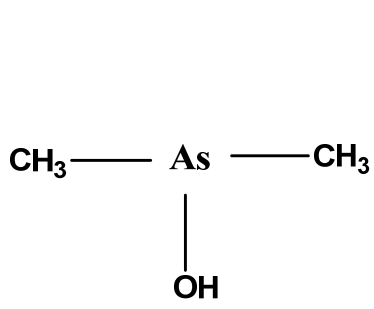

DMA(III)

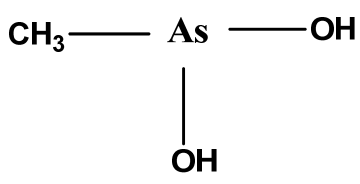

MMA(III)

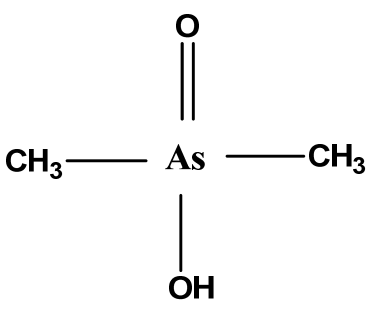

$\operatorname{DMA}(\mathbf{V})$

Figure 1. 1 Structures of the most common arsenic species in the environment

Arsenic speciation is one of the major contributing factors in the biogeochemical cycling of arsenic. The $\mathrm{pH}$ and redox environment will determine the predominant form of arsenic species present. The redox potential $\left(\mathrm{E}_{\mathrm{h}}\right)$ will determine the distribution of arsenic by oxidation states while $\mathrm{pH}$ will determine the form of oxyanion present within a particular arsenic oxidation state (Bose et al., 2002). In the environment, As(III) and $\operatorname{As}(\mathrm{V})$ do not exist as free cations but rather as oxyanions while still maintaining their +3 and +5 oxidation states (Smedley et al., 2002). Under reducing conditions (low $\mathrm{E}_{\mathrm{h}}$ ), 
$\mathrm{As}(\mathrm{III})$ is the major arsenic species and it exists as neutral $\mathrm{H}_{3} \mathrm{AsO}_{3}{ }^{0}$ species from $\mathrm{pH}$ 0-9 and has three $\mathrm{pK}_{\mathrm{a}}$ values $\left(\mathrm{pK}_{1}=9.22, \mathrm{pK}_{2}=12.13\right.$ and $\left.\mathrm{pK}_{3}=13.4\right)$ (Ko et al., 2004; Su et al., 2008). $\mathrm{H}_{2} \mathrm{AsO}_{3}^{-1}$ is the predominant species above $\mathrm{pH} 9, \mathrm{HAsO}_{3}^{-2}$ is the major species above $\mathrm{pH} 12$ and $\mathrm{AsO}_{3}^{-3}$ is the major species above $\mathrm{pH}$ 13. On the other hand, $\mathrm{As}(\mathrm{V})$ predominates under oxidizing conditions (high $\mathrm{E}_{\mathrm{h}}$ ) and exists as neutral $\mathrm{H}_{3} \mathrm{AsO}_{4}{ }^{0}$ under extreme acidic conditions $(\mathrm{pH}<2)$ and has three $\mathrm{pk}_{\mathrm{a}}$ values $\left(\mathrm{pK}_{1}=2.20, \mathrm{pK}_{2}=\right.$ 6.97 and $\left.\mathrm{pK}_{3}=11.53\right)$. In the $\mathrm{pH}$ range of 2 to $11, \mathrm{H}_{2} \mathrm{AsO}_{4}{ }^{-}$and $\mathrm{HAsO} 4^{-2}$ are the predominant species. Above $\mathrm{pH} \mathrm{11,} \mathrm{AsO}_{4}^{-3}$ is the predominant form of $\mathrm{As}(\mathrm{V})$. In the case of Fe rich and sulfide rich environments and under reducing conditions, thiolated arsenite

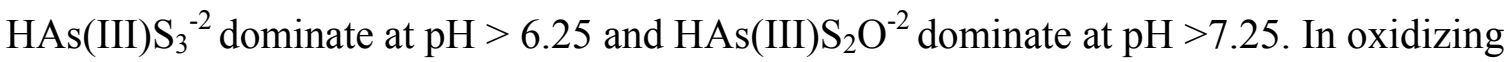
conditions, thioarsenates species dominate with $\mathrm{HAs}(\mathrm{V}) \mathrm{S}_{3} \mathrm{O}^{-2}$ present at $\mathrm{pH}<6$ and $\mathrm{As}(\mathrm{V}) \mathrm{S}_{4}{ }^{-3}$ present at higher $\mathrm{pH}$ (Couture et al., 2011). In this study the focus will be on inorganic $\mathrm{As}(\mathrm{III})$ and $\mathrm{As}(\mathrm{V})$ as these are the more predominant species in aquatic environments.

\subsubsection{Toxicity}

The toxicity of arsenic is dependent upon its chemical form and oxidation states. Generally, inorganic arsenic is considered more toxic than organic arsenicals with arsenite being more toxic than arsenate. However, recent studies have shown that MMA(III) and DMA(III) are even more toxic than inorganic As(V), MMA(V) and DMA(V).The higher toxicity of arsenite has been attributed to its strong binding affinity to sulfhydryl groups of biomolecules thereby inhibiting the activities of enzymes resulting in various harmful health effects (Sharma et al., 2009). Short and long term exposure to arsenic can result in acute and chronic health effects. Acute health effects 
include gastrointestinal discomfort, vomiting, diarrhea, bloody urine, anuria, shock, convulsions, coma, and death while chronic effects include conjunctivitis, hyperkeratosis, hyper pigmentation, cardiovascular diseases, disturbance in the peripheral vascular and nervous systems, skin lesions and blackfoot disease (Jain et al., 2000; Hughes, 2002). Chronic exposure to arsenic can also cause carcinogenic effects. Arsenic has been linked to cancer of the bladder, lungs, skin, kidney, nasal passages, liver, and prostate. The International Agency for Research on Cancer (IARC) has classified arsenic as a known human carcinogen (Group 1) based on epidemiological studies that showed an increase in skin cancer following arsenic medical treatments (IARC, 1987). Because of the severe and adverse health effects resulting from exposure to arsenic, the WHO and EPA reduced the arsenic standard in drinking water from 50 to $10 \mu \mathrm{g} \mathrm{L}^{-1}$ (Smedley et al., 2002).

\subsubsection{Arsenic Contamination in Water}

Metal contamination is a serious issue in aquatic environments and may coexist with other contaminants such as organic pollutants originating from industrial and military activities (Zhang, 2003). The human population can become exposed to arsenic through natural sources, industrial sources or from drinking water and food. The three routes of entry into the human body are: dermal, inhalation and ingestion. Dermal exposure involves arsenic absorption through the skin, however, minimal information is known regarding the arsenic chemical form or the mechanism of absorption (ATSDR, 2009). Inhalation exposure occurs from breathing in air from occupational exposure to agricultural pesticide and smelters (ATSDR, 2009). However, the primary exposure route to arsenic occurs through the ingestion of arsenic contaminated water and food (Rahman et al., 2009). 
Arsenic contamination in natural water is a worldwide problem and has become an important issue and challenge for the world engineers, scientists and even the policy makers (Choong et al., 2007). Typical arsenic concentrations in groundwater ranged from 0.5-10 $\mu \mathrm{g} / \mathrm{l}$, however elevated concentrations $(>50 \mu \mathrm{g} / \mathrm{l}$ ) are found in the groundwater of some countries. Parts of Argentina, Bangladesh, Chile, China, Hungary, India (West Bengal), Mexico, Romania, Taiwan, Vietnam and even the USA have groundwater with arsenic concentrations above $50 \mu \mathrm{g} / \mathrm{l}$ (Smedley et al., 2002). These high concentrations are a result of arsenic release from sediments such as the oxidation of arsenic pyrite minerals and/or the reductive dissolution of ferric iron hydroxide to ferrous iron consequently releasing arsenic into the water (Mandal et al., 2002). In most of these countries, groundwater is the major drinking water supply for the population. Therefore, the ingestion of high concentrations of arsenic on a daily basis poses serious health threats. About 13 million people mostly in western USA are exposed to arsenic concentrations $>10 \mu \mathrm{g} / \mathrm{L}$ (Camacho et al., 2011). In Bangladesh, up to 30-35 million people are exposed to arsenic by drinking contaminated groundwater (Smedley et al., 2002). The situation in Bangladesh is considered the largest mass poisoning in history (Das et al., 2004).

Humans are not only exposed to arsenic by drinking groundwater but also through the ingestion of arsenic contaminated food. Arsenic can be found in foods such seafood, vegetables and most importantly rice (Das et al., 2004). Seafood accounts for $60-90 \%$ of the dietary intake of arsenic but the arsenic is mostly in the organic form (Zhao et al., 2010). Arsenic in vegetables usually occurs from the irrigation of the vegetation with arsenic contaminated water (Bhattacharya et al., 2007). However, rice accounts for the 
largest contributions to the dietary intake of inorganic arsenic (Zhao et al., 2010). Rice is grown in mostly water making it more susceptible to arsenic contamination especially if it is grown in arsenic contaminated water. These elevated levels of arsenic in both drinking water and food are a major concern for millions of people around the world.

\subsubsection{Arsenic Mobility}

In general, the mobility of arsenic is dependent on processes occurring at the mineral surfaces such as precipitation, dissolution, adsorption and desorption (Bauer et al., 2006). However, the adsorption of arsenic to metal oxides particularly iron oxides plays a key role in its mobility in the environment. Iron oxides in general, whether in the bulk form or nanoparticulate form, have a strong binding affinity for both arsenite and arsenate (Jain et al., 1999). The strength of the binding and any subsequent desorption will be affected by $\mathrm{pH}$ and redox potentials. In the environment ( $\mathrm{pH} 5-8$ for natural waters), arsenite is more mobile than arsenate and it is less strongly adsorbed to the iron oxide surface (Camacho et al., 2011). Another process influencing arsenic mobility is coprecipitation which occurs after arsenic is adsorbed to the iron oxides surface. In the case of IONPs, arsenic can be trapped during the nanoparticle aggregation process and these newly formed large aggregates will precipitate out of solution thereby removing arsenic from the aqueous phase as well. Arsenic mobilization can also occur during the reductive dissolution of IONPs, a process catalyzed by iron-reducing bacteria thereby releasing arsenic back into solution (Ahmann et al., 1997). All these processes mentioned above are very significant and will affect the transport, reactivity and bioavailability of arsenic in the environment. 


\subsection{Interactions between Arsenic and Iron Oxide Nanoparticles}

It is widely known that granular or bulk iron oxide is ubiquitous and plays a significant role in the cycling of arsenic in the environment but limited knowledge is known about IONPs or NZVI interaction with arsenic. In the environment, NZVI is inevitably oxidized to iron oxide or iron hydroxide. NZVI can be oxidized to magnetite $\left(\mathrm{Fe}_{3} \mathrm{O}_{4}\right)$, ferrous hydroxide $\left[\mathrm{Fe}(\mathrm{OH})_{2}\right]$, ferric hydroxide $\left[\mathrm{Fe}(\mathrm{OH})_{3}\right]$, ferrihydrite $\left(\mathrm{Fe}_{5} \mathrm{HO}_{8} \cdot 4 \mathrm{H}_{2} \mathrm{O}\right)$, goethite $(\alpha-\mathrm{FeOOH})$ and hematite $\left(\alpha-\mathrm{Fe}_{2} \mathrm{O}_{3}\right)$ depending upon redox conditions and $\mathrm{pH}$. Each form of iron oxide shell has a different interaction with arsenic and this can influence the fate of arsenic in aquatic systems. These oxidized iron particles are expected to stay suspended or precipitate in water depending on the water chemistry and the coarsening kinetics of the particles.

The IONPs suspension can be considered as a colloidal system and it is this system that is the dominant factor controlling the speciation and mobility of metals in aquatic environment (Gustafsson et al., 1997; Bose et al., 2002). IONPs can interact with arsenic in various ways (Figure 1.2); however, adsorption is the most prominent method of interaction. IONPs has a high adsorption capacity and ligand-like coordination properties that allow arsenic to adsorb onto its oxide surface forming an inner-sphere complex (Manning et al., 2002). The IONPs have a strong affinity for both arsenite and arsenate and the retention of both arsenic species is strongly $\mathrm{pH}$ dependent (Jain et al., 1999). Kanel et al. (2005) reported that As (III) can be removed by adsorption on NZVI in a very short time (minute scale) and is strongly adsorbed over a wide range of $\mathrm{pH}$ and anion environments. The extent of removal was $88.6-99.9 \%$ in the $\mathrm{pH}$ range $4-10$ and decreased sharply at $\mathrm{pH}$ below 4 and above 10 . The $\mathrm{pH}$ dependent behavior can be 
explained by ionization of both the adsorbate and the adsorbent causing repulsion at the surface and decreasing the net As(III) adsorption. Su and Puls (2008) explained that at low $\mathrm{pH}$ surface protonation of the iron oxide occurs, and As(III) exists as a neutral $\mathrm{H}_{3} \mathrm{AsO}_{3}$ species.

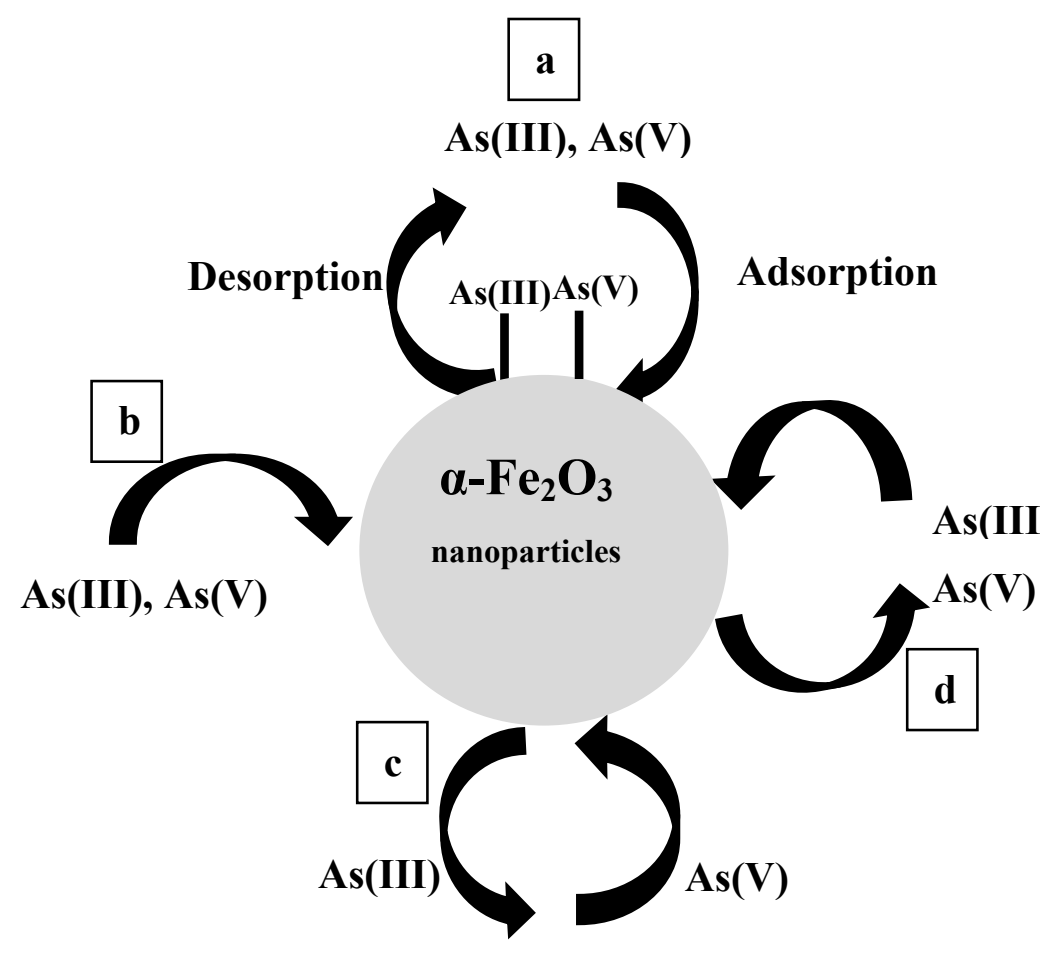

Figure 1. 2 Schematic representation of the possibly interactions of iron oxide nanoparticles with $\mathrm{As}(\mathrm{III})$ and $\mathrm{As}(\mathrm{V})$. (a) Adsorption and desorption of $\mathrm{As}(\mathrm{III})$ and $\mathrm{As}(\mathrm{V})$ to IONPs, (b) adsorption of $\mathrm{As}(\mathrm{III})$ and $\mathrm{As}(\mathrm{V})$ to IONPs followed by co-precipitation with IONPs, (c) reduction of $\mathrm{As}(\mathrm{V})$ to $\mathrm{As}(\mathrm{III})$ followed by desorption, and (d) oxidation of As(III) to As(V) followed by desorption. 
As the $\mathrm{pH}$ increases above 5, surface protonation diminishes and approaches zero at $\mathrm{pH} 7$, facilitating maximum adsorption. Electrostatic repulsion leads to decreased sorption when $\mathrm{pH}$ is above 9 since the negatively charged $\mathrm{H}_{3} \mathrm{AsO}_{3}{ }^{-}$is the dominant species and the oxide surface becomes negatively charged. Su and Puls (2001) also studied As (V) $\left(\mathrm{H}_{3} \mathrm{AsO}_{4}\right)$ adsorption onto iron oxide and showed that $\mathrm{As}(\mathrm{V})$ adsorption decreases with increasing $\mathrm{pH}$ over the $\mathrm{pH}$ range from 3 to $10 . \mathrm{H}_{2} \mathrm{AsO}_{4}{ }^{-}$is the predominant species at $\mathrm{pH} 3-6$, and is the major species being adsorbed due to the iron oxide surfaces having a net positive charge in this $\mathrm{pH}$ range. Therefore $\mathrm{pH}$ plays a key role in the adsorption and desorption of arsenic subsequently affecting arsenic's mobility in the environment.

Iron oxide nanoparticles' strong adsorption characteristics make it more applicable to contaminant remediation. However the chemical interactions between IONPs and arsenic need to be evaluated. Although it is well known that adsorption and desorption are the main interactions between IONPs and arsenic, not much work has been done on the possible species transformation when arsenic is adsorbed or in contact with IONPs. Both arsenic and iron are redox sensitive and can undergo redox transformation in the environment either microbially or photochemically (Masscheleyn et al., 1991). However, once arsenic is adsorbed to the nanoparticle surface, the redox transformation of iron can somehow influence the redox transformation of arsenic. Therefore, arsenic speciation studies in the presence of IONPs need to be evaluated. The transformation of arsenic species will depend on various factors such as the redox potential, the iron species present, $\mathrm{pH}$, the presence of microbes, and photochemical effect. 
Few research groups have reported speciation of arsenic in the presence of iron nanoparticles. Kanel et al. (2005) found that As(III) near or in contact with the corroding NZVI surface can be oxidized to As(V). In addition, Su and Puls (2001) reported that there is a strong evidence for partial oxidation of $\mathrm{As}(\mathrm{III})$ to form $\mathrm{As}(\mathrm{V})$ in zerovalent iron solutions, whereas there is no evidence for significant reduction of $A s(V)$ under the same experimental conditions. Bose et al. (2002) reported that there is possible reduction of $\mathrm{As}(\mathrm{V})$ to $\mathrm{As}(\mathrm{III})$ during the dissolution of $\mathrm{As}(\mathrm{V})$ - iron oxide precipitate thereby releasing arsenic (V) and iron (II) in solution, then followed by reduction of arsenic (V) to arsenic (III) in the dissolved phase. The transformation process of arsenic in the presence of IONPs and how often it occurs is unclear and a more thorough understanding is needed.

The transformation and fate of arsenic is dependent upon the colloidal stability of the IONPs. Colloidal stability can be defined as the ability of a particle dispersion to resist aggregation for a specific time (Phenrat et al., 2007). Colloidal particles frequently aggregate to larger particles in circumneutral water and can settle out of solution. Therefore, aggregation is an important factor to consider because the dispersion behavior of nanoparticles will affect their reactivity in environmental settings (Gilbert et al., 2007). Aggregation can be beneficial by immobilizing arsenic through the co-precipitation process. The process usually involves arsenic adsorbing to the nanoparticles and getting trapped inside an aggregate during particle growth. The aggregate will subsequently precipitate out of solution with the trapped arsenic thereby causing a reduction of arsenic in the aqueous phase. On the other hand aggregation can be ineffective as the nanoparticles grow larger in size causing a reduction in the surface area thereby lowering the absorption capacity. In recent years, the application of stabilizers to modify the 
nanoparticle surface has emerged as a method to prevent aggregation and prolong colloidal stability. Stabilizers are soluble polymers or surfactants that are attached to surface of the nanoparticles providing strong interparticle electrostatic and steric repulsion that will overcome the van der waals attraction and magnetic forces that causes aggregation (He et al., 2007). Schrick et al. (2004) and Yang et al (2007) reported that nanoiron modified with polyacrylic acid (PAA) formed stable and mobile suspensions. Kanel et al. (2007) reported the use of tween 20, He and Zhao (2007) reported the use of carboxymethyl cellulose and Terifarri et al. (2008) used guar gum as stabilizers to prevent aggregation. Although stabilizers are effective in enhancing colloidal stability, they may interfere with the interaction between arsenic and the nanoparticle surface. Therefore, understanding the colloidal behavior and aggregation of IONPs is key to determine its role on the transport and transformation of arsenic in the environment.

Studies on the interaction of iron nanoparticles with arsenic are relatively new but are of utmost importance. With the increase interest in the use of IONPs or NZVI for remediation application, and the high toxicity and prevalence of arsenic in the environment, it is crucial that we evaluate the interaction between arsenic and IONPs. Many uncertainties arise when we try to understand the effects of IONPs on arsenic speciation, mobility and fate in the environment. Some of these include: 1) how does IONPs exist in aquatic environments in regards to their particle size, aggregation and stability in the presence of environmental factors such as organic matter and salinity? 2) How do the interactions between IONPs and arsenic affect their transport in the environment? 3) What is the role and how much influence does IONPs have on the transformation of arsenic? In this study, we seek to answer these questions and determine 
the extent at which iron nanoparticles influence the biogeochemical cycling of arsenic. The information we gather is necessary and crucial for the assessment of the prospective use of iron nanomaterials in clean up strategies for arsenic and other toxic metals. This information will further our knowledge on the biogeochemical cycling of arsenic and assist in understanding the behavior of nanoparticles in the environment. In addition, through this study, we can have an understanding on how natural and engineered nanoparticles might play a role in the cycling of arsenic and other metals in the environment.

\subsection{Research Objectives and Hypothesis}

The primary purpose of this research is to study the effects of iron oxide nanoparticles on the fate, mobility and transformation of arsenic in aquatic environment. To achieve this goal, the following objectives will be investigated:

\subsubsection{To Prepare a Stable IONPs Dispersion without the Use of Surface Modifications.}

The goal here was to develop an efficient method to disperse and stabilize bare iron oxide nanoparticles without the use of surface modifications and to determine the stability of the dispersion. Experiments were designed to disperse commercial IONPs into nanoparticulate size ranges using bath sonication, probe sonication and ultrasonication and to determine the stability of the dispersion after being exposed to environmentally relevant conditions. This work was aimed to mimic the way IONPs may exist in the environment and to determine their environmental behavior. The findings will ultimately give an indication on how nanoparticles might exist in the environment and how their properties will influence their interaction with metal contaminants. 
Hypothesis: It was hypothesized that IONPs can be dispersed and stable for a prolonged period of time under environmental conditions without the use of surface modifications. The hypothesis was formed on the basis that IONPs powder can form dispersions in water by mechanically breaking down the particles from large aggregates to smaller particles. However, breaking the IONPs apart does not guarantee stability. The particle size, concentration of the suspended particles, the surface chemistry of the particle, $\mathrm{pH}$, ionic strength and solution composition will determine the level of stability and how fast the nanoparticles aggregate. These dispersions should be prepared without stabilizers modifying the nanoparticle surface thereby enhancing the stability either electrostatically or sterically. These stabilizers themselves can affect the reactivity of the nanoparticle and limit the interactions between the nanoparticle surface and metal contaminants.

\subsubsection{Quantification of the Interactions (kinetics and thermodynamics) of}

\section{Engineered Iron Oxide Nanoparticles and Iron Oxide Aggregates with Different}

\section{Arsenic Species.}

The goal here was to investigate the adsorption and retention of arsenic to iron nanoparticles or aggregates under environmental conditions. The effects of particle size on the adsorption process were investigated while acquiring kinetic and thermodynamic data. This step is initial but will provide important data that will give us a solid basis for studying the environmental fate and transformation of arsenic.

Hypothesis: It was hypothesized that iron oxides whether in the nanoparticulate form or colloidal aggregate will adsorb arsenic onto their surfaces, however, the adsorption process will be different depending on the size of the iron oxide particles and the type of sorbed arsenic species. The hypothesis was formed on the basis that iron oxides in 
general are known to have a strong affinity for adsorbing heavy metals. Therefore, arsenic will readily adsorbs onto iron oxides surfaces and the extent of adsorption will vary depending on the arsenic's oxidation state. In terms of the particle size, the smaller the iron oxide particle the higher the efficiency of adsorption. Nanoparticles tend to have high surface area and larger amounts of binding sites thereby accommodating higher amounts of arsenic adsorption onto their surface compared to colloidal aggregates.

\subsubsection{Quantification of the Key Transformation Processes of Arsenic in the presence of Iron Oxide Nanoparticles and Iron Oxide Aggregates.}

The third objective determined the key processes of transformation of arsenic and the factors that could possibly influence arsenic speciation. The information gathered will give us a better understanding of the biogeochemical cycling of arsenic since speciation determines the degree of mobility of arsenic in the environment.

Hypothesis: The transformation between arsenic species can be affected by varying chemical reactivity and environmental conditions. It was hypothesized that the transformation such as $\mathrm{As}(\mathrm{III})$ oxidation to $\mathrm{As}(\mathrm{V})$ or $\mathrm{As}(\mathrm{V})$ reduction to $\mathrm{As}(\mathrm{III})$ would behave differently in the presence of IONPs and iron oxide aggregates. It is widely known that aqueous phase chemical and direct microbial catalyzed redox transformations of arsenic are responsible for speciation. However, other factors such as mineral phases can play a significant role in the transformation process. Once arsenic adsorbs onto these iron oxide surfaces, it might undergo surface mediated reduction or oxidation reactions. 


\subsection{List of References}

Ahmann D, Krumholz LR, Hemond HF, Lovley DR, Morel FMM. Microbial Mobilization of Arsenic from Sediments of the Aberjona Watershed. Environ Sci Technol 1997; 31: 2923-2930.

ATSDR. Case studies in environmental medicine. 2009: Agency for Toxic Substances and Disease Registry: http://www.atsdr.cdc.gov/csem/arsenic/docs/arsenic.pdf.

Bauer M, Blodau C. Mobilization of arsenic by dissolved organic matter from iron oxides, soils and sediments. Sci Total Environ 2006; 354: 179-190.

Bhattacharya P, Welch AH, Stollenwerk KG, McLaughlin MJ, Bundschuh J, Panaullah G. Arsenic in the environment: Biology and Chemistry. Sci Total Environ 2007; 379: 109-120.

Bissen M, Frimmel FH. Arsenic - a review. Part I: Occurrence, toxicity, speciation, mobility. Acta Hydrochim Hydrobiol 2003; 31: 9-18.

Bose P, Sharma A. Role of iron in controlling speciation and mobilization of arsenic in subsurface environment. Water Res 2002; 36: 4916-4926.

Camacho LM, Gutierrez M, Alarcon-Herrera MT, Villalba MdL, Deng S. Occurrence and treatment of arsenic in groundwater and soil in northern Mexico and southwestern USA. Chemosphere 2011; 83: 211-225.

Carabante I, Grahn M, Holmgren A, Kumpiene J, Hedlund J. Adsorption of As (V) on iron oxide nanoparticle films studied by in situ ATR-FTIR spectroscopy. Colloid Surface A 2009; 346: 106-113.

Chakraborti D. Arsenic: Occurrence in Groundwater. Encyclopedia of Environmental Health. 1. Elsevier, Burlington, 2011, pp. 165-180.

Choong TSY, Chuah TG, Robiah Y, Koay FLG, Azni I. Arsenic toxicity, health hazards and removal techniques from water: an overview. Desalination 2007; 217: 139166.

Couture RM, Van Cappellen P. Reassessing the role of sulfur geochemistry on arsenic speciation in reducing environments. J hazard mater 2011; 189: 647-652.

Cundy AB, Hopkinson L, Whitby RLD. Use of iron-based technologies in contaminated land and groundwater remediation: A review. Sci Total Environ 2008; 400: 42-51.

Das HK, Mitra AK, Sengupta PK, Hossain A, Islam F, Rabbani GH. Arsenic concentrations in rice, vegetables, and fish in Bangladesh: a preliminary study. Environment International 2004; 30: 383-387. 
Gilbert B, Lu G, Kim CS. Stable cluster formation in aqueous suspensions of iron oxyhydroxide nanoparticles. J Colloid Interface Sci 2007; 313: 152-159.

Gustafsson O, Gschwend PM. Aquatic colloids: concepts, definitions, and current challenges. Limnol and Oceanogr 1997; 42: 519-528.

He F, Zhao D. Manipulating the Size and Dispersibility of Zerovalent Iron Nanoparticles by Use of Carboxymethyl Cellulose Stabilizers. Environ Sci Technol 2007; 41: 6216-6221.

He YT, Wan J, Tokunaga T. Kinetic stability of hematite nanoparticles: the effect of particle sizes. J Nanopart Res 2008; 10: 321-332.

Hughes MF. Arsenic toxicity and potential mechanisms of action. Toxicol Lett 2002; 133: $1-16$.

IARC. Arsenic and arsenic compounds (Group I). In: IARC monographs on the evaluation of the carcinogenic risks to humans. Supplement 7. 1987: International Agency for Research on Cancer.

Jain A, Raven KP, Loeppert RH. Arsenite and Arsenate Adsorption on Ferrihydrite: Surface Charge Reduction and Net OH- Release Stoichiometry. Environ Sci Technol 1999; 33: 1179-1184.

Jain CK, Ali I. Arsenic: occurrence, toxicity and speciation techniques. Water Res 2000; 34: 4304-4312.

Jambor JL, Dutrizac JE. Occurrence and Constitution of Natural and Synthetic Ferrihydrite, a Widespread Iron Oxyhydroxide. Chem Rev 1998; 98: 2549-2585.

Kanel SR, Manning B, Charlet L, Choi H. Removal of arsenic(III) from groundwater by nanoscale zero-valent iron. Environ Sci Technol 2005; 39: 1291-8.

Karn B, Kuiken T, Otto M. Nanotechnology and in situ remediation: a review of the benefits and potential risks. Environ Health Perspect 2009; 117: 1813-31.

Ko I, Kim J-Y, Kim K-W. Arsenic speciation and sorption kinetics in the As-hematitehumic acid system. Colloids Surf 2004; 234: 43-50.

Kohlmeyer U, Jantzen E, Kuballa J, Jakubik S. Benefits of high resolution IC-ICP-MS for the routine analysis of inorganic and organic arsenic species in food products of marine and terrestrial origin. Anal Bioanal Chem 2003; 377: 6-13.

Liu Y, Lowry GV. Effect of Particle Age (Fe0 Content) and Solution pH On NZVI Reactivity: H2 Evolution and TCE Dechlorination. Environ Sci Technol 2006; 40: 6085-6090. 
Liu Y, Majetich SA, Tilton RD, Sholl DS, Lowry GV. TCE Dechlorination Rates, Pathways, and Efficiency of Nanoscale Iron Particles with Different Properties. Environ Sci Technol 2005; 39: 1338-1345.

Mandal BK, Suzuki KT. Arsenic round the world: a review. Talanta 2002; 58: 201-235.

Manning BA, Hunt ML, Amrhein C, Yarmoff JA. Arsenic(III) and Arsenic(V) Reactions with Zerovalent Iron Corrosion Products. Environ Sci Technol 2002; 36: 54555461 .

Martin JE, Herzing AA, Yan W, Li X-q, Koel BE, Kiely CJ, et al. Determination of the Oxide Layer Thickness in Core-Shell Zerovalent Iron Nanoparticles. Langmuir 2008; 24: 4329-4334.

Masscheleyn PH, Delaune RD, Patrick WH. Effect of redox potential and $\mathrm{pH}$ on arsenic speciation and solubility in a contaminated soil. Environ Sci Technol 1991; 25: 1414-1419.

Phenrat T, Saleh N, Sirk K, Tilton RD, Lowry GV. Aggregation and Sedimentation of Aqueous Nanoscale Zerovalent Iron Dispersions. Environ Sci Technol 2007; 41: 284-290.

Ponder SM, Darab JG, Mallouk TE. Remediation of Cr(VI) and Pb(II) Aqueous Solutions Using Supported, Nanoscale Zero-valent Iron. Environ Sci Technol 2000; 34: 2564-2569.

Quinn J, Geiger C, Clausen C, Brooks K, Coon C, O'Hara S, et al. Field Demonstration of DNAPL Dehalogenation Using Emulsified Zero-Valent Iron. Environ Sci Technol 2005; 39: 1309-1318.

Rahman MM, Ng JC, Naidu R. Chronic exposure of arsenic via drinking water and its adverse health impacts on humans. Environ Geochem Health 2009; 31: 189-200.

Rebodos RL, Vikesland PJ. Effects of Oxidation on the Magnetization of Nanoparticulate Magnetite. Langmuir 2010; 26: 16745-16753.

Rossman TG. Mechanism of arsenic carcinogenesis: an integrated approach. Mutat. Res., Fundam. Mol. Mech. Mutagen. 2003; 533: 37-65.

Schwertmann U, Cornell RM. Iron Oxides in the Laboratory: Preparation and Characterization. Weinheim: Wiley, 2000.

Sharma VK, Sohn M. Aquatic arsenic: Toxicity, speciation, transformations, and remediation. Environ Int 2009; 35: 743-759.

Shipley H, Engates K, Guettner A. Study of iron oxide nanoparticles in soil for remediation of arsenic. J Nanopart Res 2010: 1-11-11. 
Smedley PL, Kinniburgh DG. A review of the source, behaviour and distribution of arsenic in natural waters. Appl Geochem 2002; 17: 517-568.

Su C, Puls RW. Arsenate and Arsenite Sorption on Magnetite: Relations to Groundwater Arsenic Treatment Using Zerovalent Iron and Natural Attenuation. Water, Air, Soil Poll 2008; 193: 65-78.

Suess E, Planer-Friedrich B. Thioarsenate formation upon dissolution of orpiment and arsenopyrite. Chemosphere 2012; 89: 1390-1398.

Sun Y-P, Li X-q, Cao J, Zhang W-x, Wang HP. Characterization of zero-valent iron nanoparticles. Adv Colloid Interface Sci 2006; 120: 47-56.

Taylor KG, Konhauser KO. Iron in Earth Surface Systems: A Major Player in Chemical and Biological Processes. Elements 2011; 7: 83-87.

Theron J, Walker JA, Cloete TE. Nanotechnology and water treatment: Applications and emerging opportunities. Crit Rev Microbiol 2008; 34: 43-69.

Turk T, Alp I, Deveci H. Adsorption of As(V) from water using nanomagnetite. J Environ Eng 2010; 136: 399-404.

U.S. EPA. Engineered approaches to in situ bioremediation of chlorinated solvents: fundamentals and field applications. 2000: EPA 542-R-00-008. Washington, DC: U.S. Environmental Protection Agency.

U.S. EPA. Nanotechnology for Site Remediation Fact Sheet. 2008: EPA 542-F-08-009. Washington, DC: U.S. Environmental Protection Agency. Available: http://www.epa.gov/tio/download/remed/542-f-08-009.pdf.

Wang C-B, Zhang W-x. Synthesizing Nanoscale Iron Particles for Rapid and Complete Dechlorination of TCE and PCBs. Environ Sci Technol 1997; 31: 2154-2156.

WHO. Arsenic in Drinking-water. 2011: World Health Organization; http://www.who.int/water_sanitation_health/dwq/chemicals/arsenic.pdf.

Yan W, Herzing AA, Kiely CJ, Zhang W-x. Nanoscale zero-valent iron (nZVI): Aspects of the core-shell structure and reactions with inorganic species in water. J Contam Hydrol 2010; 118: 96-104.

Yunmei Y, Yongxuan Z, Williams-Jones AE, Zhenmin G, Dexian L. A kinetic study of the oxidation of arsenopyrite in acidic solutions: implications for the environment. Appl Geochem 2004; 19: 435-444.

Zhang W-x. Nanoscale Iron Particles for Environmental Remediation: An Overview. J Nanopart Res 2003; 5: 323-332. 
Zhang W, Cai Y, Tu C, Ma LQ. Arsenic speciation and distribution in an arsenic hyperaccumulating plant. Sci Total Environ 2002; 300: 167-177.

Zhao FJ, McGrath SP, Meharg AA. Arsenic as a food chain contaminant: mechanisms of plant uptake and metabolism and mitigation strategies. Annu Rev of Plant Biol 2010; 61: 535-559. 


\section{CHAPTER II}

Dispersion and stability of bare hematite nanoparticles: effect of dispersion tools, nanoparticle concentration, humic acid and ionic strength

The work described in this chapter has been published in the Science of the Total

Environment 2012; 419: 170-177 


\subsection{Abstract}

The aggregation and sedimentation of iron oxide nanoparticles (IONPs) can significantly affect the mobility and reactivity of IONPs and subsequently influence the interaction between IONPs and environmental contaminants. Dispersing bare IONPs into a stable suspension within nanoscale range is an important step for studying the interaction of IONPs with contaminants (e.g., toxic metals). In this study, different techniques to disperse bare IONPs (vortex, bath sonication and probe ultrasonication) and the effects of important environmental factors such as dissolved organic matter and ionic strength on the stability of IONPs dispersions were investigated. Vortex minimally dispersed IONPs with hydrodynamic diameter outside the "nano-size range" (6982400nm). Similar to vortex, bath sonication could not disperse IONPs efficiently. Probe ultrasonication was more effective at dispersing IONPs (50\% or more) with hydrodynamic diameters ranging from 120-140 nm with minimal changes in size and sedimentation of IONPs for a prolonged period of time. Over the course of 168 hours, considerable amounts of IONPs remained dispersed in the presence and absence of low ionic strength $(0.1 \mathrm{mM}$ of $\mathrm{NaCl})$ and $100 \mathrm{mg} / \mathrm{L}$ of humic acid (HA). These results indicate that IONPs can be broken down efficiently into "nanosize range" by probe ultrasonication and a degree of stability can be achieved without the use of synthetic modifiers to enhance colloidal stability. This dispersion tool could be used to develop a laboratory method to study the adsorption mechanism between dispersed bare IONPs and toxic contaminants. 


\subsection{Introduction}

Groundwater pollution by toxic heavy metals has become an important issue globally and the development of inexpensive remediation technologies to clean up water is crucial. Nanotechnology has emerged as one of the leading technologies for cleaning up polluted sites. Currently, nanotechnology is widely studied and has shown considerable growth in the remediation of contaminants in ground and surface waters. Nanoscale zerovalent iron (NZVI) is considered as the first generation nanoscale environmental technologies and has the potential to remove a wide range of pollutants (Sun et al., 2006). NZVI has shown significant applications in groundwater remediation of chlorinated organic compounds such as trichloroethylene, organochlorine pesticides, polychlorinated biphenyls as well as remediation of toxic metals (Phenrat et al., 2007). In the past few years, a variety of iron oxide nanoparticles (IONPs) have been studied for environmental remediation purposes. These IONPs behave similarly to NZVI when used as treatment for contaminated sites and are frequently used as a model system for understanding aggregation behavior (He et al., 2008).

The growing interest in engineered NZVI and IONPs for groundwater remediation is attributed to the large surface area $\left(25-54 \mathrm{~m}^{2} \mathrm{~g}^{-1}\right)$, highly reactive surface sites and high in-situ reactivity (Phenrat et al., 2007; Theron et al., 2008). The possibility of in situ remediation results in shorter remediation time and low cost, as nanoscale metal particles are applied directly to contaminated sites (Wang et al., 1997; He et al., 2007). Despite the advantages, NZVI and IONPs have one major limitation that can be detrimental to their use as effective groundwater treatment. Studies have shown rapid agglomeration of NZVI and IONPs to form large aggregates that will sediment (He et al., 
2007). The surface properties of NZVI and IONPs influence their aggregation. Once in aquatic environment, the aggregation of nanoparticles results from the interaction between nanoparticles surface and water components and is strongly influenced by several factors such as salinity, solution composition, surface chemistry, concentration of suspended particles, and the $\mathrm{pH}$ especially where the nanoparticles is approaching the pH of point zero charge (PZC) (Mylon et al., 2004; Yang et al., 2007; Baalousha, 2009; Hu et al., 2010). The particle-particle interactions are the driving forces for aggregation and deposition of nanoparticles although other interactions such as steric, magnetic and hydration forces can also play an important role (Petosa et al., 2010). The DerjaguinLandau-Verwey- Overbeek (DLVO) theory describes colloidal stability and can be used to explain the stability of nanoparticles in aquatic environment. The DLVO theory states that the stability of nanoparticles can be explained by the sum (total interaction energy) of van der Waals and electric double layer interactions (Deryagin et al., 1941; Verwey, 1947; Verwey et al., 1948; Petosa et al., 2010). The total interaction energy is experienced by a nanoparticle when it is approaching another particle and this energy determines whether the net interaction between particles are repulsive or attractive (Zhang et al., 2008) . In addition, the Hamaker theory is an important component in the DLVO theory as the Hamaker constant relates the interatomic van der Waals interaction to the total van der Waals interaction (Petosa et al., 2010).

Consequently aggregation and sedimentation can significantly alter the mobility of the nanoparticles in aquatic environment and reduce the efficacy of using these nanoparticles for remediation purposes (Sun et al., 2007; Kim et al., 2009). Therefore, stable dispersions of nanoparticles are critical for efficient sorption of pollutants. In 
recent years, there is noticeably increase in the application of modifiers, e.g., soluble polymers and surfactants, to alter the surface of nanoparticles thus preventing aggregation (Schrick et al., 2004; Kanel et al., 2007; Yang et al., 2007). Several researchers have reported the use of modifiers that are capable of reducing NZVI and IONPs aggregation (Schrick et al., 2004; He et al., 2007; Kanel et al., 2007; Yang et al., 2007; Tiraferri et al., 2008). Although modifiers can enhance colloidal stability, they can be expensive, have adverse effect on the environment and alter the surface of the nanoparticles, consequently affecting the fate and transport of nanoparticles and their interaction with contaminants (Tiraferri et al., 2008). Specifically, modifiers could affect the sorption and desorption of contaminants, introduce additional mass transfer limitations of contaminants to active surface sites, and could decrease the reaction rate of the reactions that could be taking place at the surface (Phenrat et al., 2009). Phenrat el al. (2009) reported a decrease in dechlorination rate of trichloroethylene nonlinearly with increase surface modifiers on $\mathrm{Fe}^{0} / \mathrm{Fe}_{3} \mathrm{O}_{4}$ nanoparticles. In order to better understand the environmental fate and transport of "true" IONPs and the true representation of how the IONPs interact with other contaminants, development of a method to disperse and stabilize bare/uncoated IONPs at relevant environmental conditions over a prolonged period of time is necessary.

The goal of this research was to develop a method to disperse bare IONPs and to determine IONPs stability after being dispersed and exposed to environmentally relevant conditions ( $\mathrm{pH}$ 6-8 and in the presence of natural organic matter and ionic strength). This work was not aimed to disperse large quantity of IONPs for real field practice, but aimed to disperse IONPs in a laboratory setting in order to provide a tool to prepare a 
uniformly dispersed suspension. The purpose of preparing such a bare IONPs

suspension was to estimate how nanomaterials might exist in the environment and this in turn will provide an in-depth understanding of how nanomaterials will behave in the environment with respect to the fate and transformation of contaminants. As one of the most widespread and relatively thermodynamically stable iron oxide in the environment, hematite nanoparticles are a good model system for understanding aggregation of IONPs (Schwertmann et al., 2000). This study investigated and compared different mechanical methods to disperse hematite nanoparticles without the use of modifiers.

\subsection{Procedures and Methods}

\subsubsection{Materials and Chemicals}

Commercial iron oxide nanoparticles $\left(\alpha-\mathrm{Fe}_{2} \mathrm{O}_{3}, 98 \%\right.$ purity and $50 \mathrm{~m}^{2} / \mathrm{g}$ specific surface area, primary particle diameter from 20 to $50 \mathrm{~nm}$ ) were purchased from Nanostructured and Amorphous Materials (Houston, Texas). Humic acid (HA) was obtained from Acros Organic (New Jersey, USA) and sodium chloride was purchased from Fisher Scientific (New Jersey, USA). All materials were suspended or dissolved in nanopure $18.2 \mathrm{M} \Omega$ water produced from a nanopure diamond lab water system (Barnstead Thermolyne Corporation, Dubuque, IA) and prepared in Corning $50 \mathrm{~mL}$ polypropylene centrifuge tubes.

\subsubsection{Instrumentation}

Iron oxide nanoparticles were dispersed using a Fisher Scientific touch mixer model 232 (Pittsburgh, PA), Branson ultrasonic model 1510 (Danbury, CT) and Fisher Scientific sonic dismembrator model 100 (Pittsburg, PA). For particle size monitoring using dynamic light scattering (DLS), a Malvern Zetasizer Nano-ZS (Westborough, 
MA.) was employed. The hydrodynamic diameters reported in this study represent the average particle diameter "z-average" intensity peak as a function of size. Zeta potential measurements were also made using the Malvern Zetasizer Nano-ZS. Total iron concentration was monitored using a graphite furnace atomic absorption spectrometer (GFAAS) (Perkin Elmer, model Aanalyst 600). The pH measurements were made using a Fisher Scientific accumet Research $\mathrm{AR} 15 \mathrm{pH} / \mathrm{mV} /{ }^{\circ} \mathrm{C}$ Meter.

\subsubsection{Experimental procedures}

\subsubsection{Effect of Dispersion Techniques on IONPs Stability}

Dispersion of iron oxide nanoparticles. Stock suspension (40 mL) of $\alpha-\mathrm{Fe}_{2} \mathrm{O}_{3}$

nanoparticles was prepared by mixing the required amount of $\alpha-\mathrm{Fe}_{2} \mathrm{O}_{3}$ nanoparticles and nanopure water to make a concentration of $500 \mathrm{mg} / \mathrm{L}$ of $\alpha-\mathrm{Fe}_{2} \mathrm{O}_{3}$ (IONPs). The stock suspension was dispersed using a vortex, bath sonication or probe ultrasonication to investigate which technique can provide sufficient power to disperse the nanoparticles. For vortexing, the stock suspension was vortexed for 20 minutes at the highest speed (speed 10). For bath sonication (power density of $0.0370 \mathrm{~W} / \mathrm{mL}$ ), the stock suspension was sonicated for 30 minutes. For ultrasonication, the following probe power and sonication times were investigated: probe power level 3 (power density of $0.275 \mathrm{~W} / \mathrm{mL}$ ) and level 6 (power density of $0.55 \mathrm{~W} / \mathrm{mL}$ ) at 5, 20 and 60 minutes of sonication for each power level. Immediately following sonication $20 \mathrm{~mL}$ of IONPs suspensions (each containing $10,30,50,75,100$ and $200 \mathrm{mg} / \mathrm{L}$ of $\alpha-\mathrm{Fe}_{2} \mathrm{O}_{3}$ ) were prepared by subsequent dilution of the stock suspension. Time dependent hydrodynamic diameters of the nanoparticles were measured in triplicate at $0,1,3,5,8,24,48,72$ and 168 hours with 
all sizes reported as mean \pm standard deviation. Suspensions had $\mathrm{pH}$ ranging from 6.2 to 7.60. The drift in $\mathrm{pH}$ was a result of the suspensions not being buffered.

Iron analysis. Total iron concentration in the 10, 30, 50, 75, 100 and $200 \mathrm{mg} / \mathrm{L}$ IONPs suspensions was analyzed using GFAAS to monitor the sedimentation of IONPs over time. Before iron analysis, IONPs suspension underwent acid digestion in 50\% nitric acid for 20 minutes at $95 \pm 5^{\circ} \mathrm{C}$.

\subsubsection{Effect of Humic Acid (HA) and Ionic Strength on IONPs Stability}

A $500 \mathrm{mg} / \mathrm{L}$ stock suspension $(40 \mathrm{~mL})$ of $\alpha-\mathrm{Fe}_{2} \mathrm{O}_{3}$ nanoparticles was prepared and dispersed using probe ultrasonication for 20 minutes at power level 6 . After probe sonication concentrations of IONPs $(10,30,50,75,100$ and $200 \mathrm{mg} / \mathrm{L})$ were prepared by subsequent dilution of the stock suspension and spiked with appropriate amount of HA to make a concentration of $100 \mathrm{mg} / \mathrm{L} \mathrm{HA}$. For the effect of ionic strength, the diluted IONPs suspensions were spiked with the appropriate amount of $\mathrm{NaCl}$ to make a concentration of $0.1,10$ and $100 \mathrm{mM} \mathrm{NaCl}$. In addition, the stability of IONPs was also investigated in the presence of both $\mathrm{HA}(100 \mathrm{mg} / \mathrm{L})$ and $\mathrm{NaCl}(0.1 \mathrm{mM})$. For these experiments, DLS measurements and iron quantification were made at $0,1,3,5,8,24$, 48, 72 and 168 hours. Zeta potential measurements were made while investigating the effect of HA alone on the aggregation behavior of IONPs. Zeta potential measurements could indicate whether HA is able to adsorb to IONPs resulting in a change in charge on the IONPs surface. 


\subsection{Results and Discussion}

\subsubsection{Effect of Dispersion Techniques on IONPs Size Distribution}

Commercial grade nanoparticles are obtained as agglomerates and the breakage of IONPs to its primary particle size is an important step to prepare a stable dispersion. There was difficulty dispersing IONPs by vortex as most of the nanoparticles remained aggregated at the bottom of the sample containers and any dispersed particles quickly precipitated. At $t_{0}$ (measured immediately following vortex) the average particle diameters ranged from 698-2400 $\mathrm{nm}$ with smaller sizes observed with increasing time as the larger aggregates sediment leaving the smaller ones remaining in suspension (Figure 2.1a). These measured hydrodynamic diameters are significantly larger than the 20-50 $\mathrm{nm}$ range reported by the manufacturer. Depending on the concentration of IONPs, the hydrodynamic diameters vary in size. The dilute dispersions (10, 30 and $50 \mathrm{mg} / \mathrm{L})$ had the largest particle size at $t_{0}$ while the higher concentrations $(75,100$ and $200 \mathrm{mg} / \mathrm{L})$ had smaller sizes. This difference is likely due to a higher rate of collision between particles in concentrated dispersions thus resulting in higher aggregation rate and subsequent sedimentation of the nanoparticles (Baalousha et al., 2008; Baalousha, 2009). Therefore, vortex lacks the capability of breaking up IONPs powder and does not sufficiently disperse IONPs. In addition, dispersion by bath sonication was also investigated but rapid sedimentation immediately following sonication (data not shown) limited its capability to disperse IONPs.

Probe ultrasonication was employed as a dispersion tool and IONPs were more uniformly dispersed with smaller hydrodynamic diameter. Figure $2.1 \mathrm{~b}$ shows the change in hydrodynamic diameter with time for different concentrations of IONPs dispersed 
using ultrasonic probe for 20 minutes at power level 6 (power density of $0.55 \mathrm{~W} / \mathrm{mL}$ )

Other conditions such as varying the sonication time and power density ( 5 and 60 minutes of sonication at both power level 3 and 6) had similar results (data not shown).
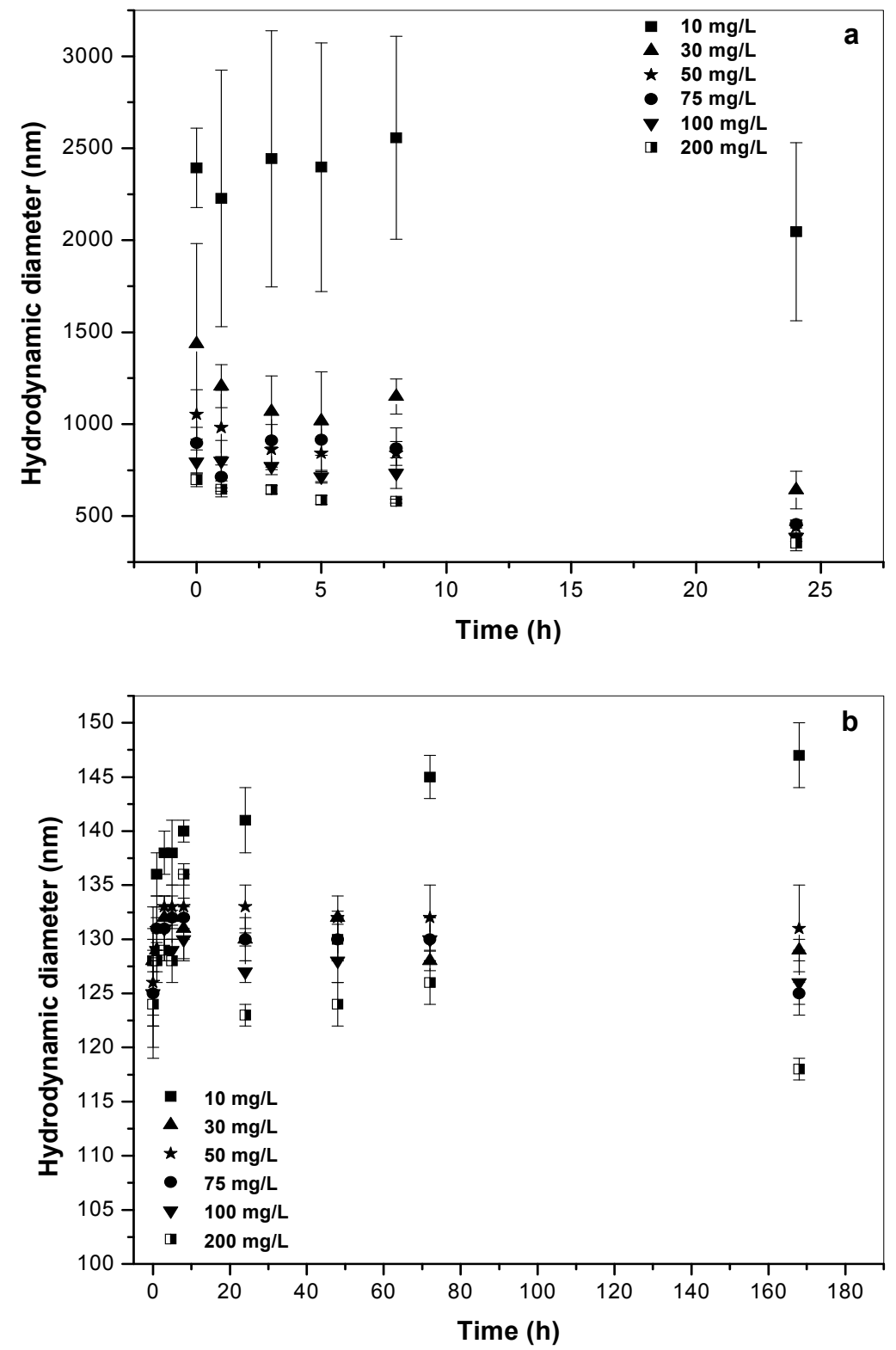

Figure 2. 1 The time dependent hydrodynamic diameter of 10, 30, 50, 75, 100 and 200 $\mathrm{mg} / \mathrm{L}$ IONPs dispersed by (a) vortex for 20 minutes at power level 10 and (b) ultrasonic probe for 20 minutes at power level 6. 
Regardless of IONPs concentration, probe power density and time of sonication $(5,20$ or 60 minutes), the particle diameter ranged between $124-128 \mathrm{~nm}$ at $\mathrm{t}_{0}$ with minimal changes in size over a period of 168 hours. This range of particle size (124-128 nm) was $2-5$ times larger than the manufacturers reported primary particle diameter $(20-50 \mathrm{~nm})$ and it was slightly greater than the size defined as nanoparticles $(<100 \mathrm{~nm})$. For the purpose of easy discussion, we name these particles as nanoparticles in this paper. The ultrasonic probe was a more powerful tool to disperse IONPs when compared to vortex and bath sonication. The difference in dispersion is attributed to the fact that the ultrasonic probe is directly inserted in the sample and it has greater power than the other dispersion methods (Santos et al., 2007). The acoustic waves imparted by ultrasonication is effective in dispersing IONPs due to the transient cavitation and acoustic streaming that can possibly redefine the shape and structure of nanoparticles and change the surface morphology (Suslick et al., 1999). Specifically, acoustic cavitation gave rise to cavitation cycles that involves the nucleation and collapsing of micro-bubbles creating micro "hot spots" where nanoparticles experience extreme conditions such as high temperatures and pressures. These conditions result in the breakage of aggregated nanoparticles and a more uniform dispersion in liquids (Suslick et al., 1999; Mandzy et al., 2005).

Although probe ultrasonication broke up the nanoparticles extensively, it could not break down IONPs to the primary particle diameter due to the possible aggregation of the particles during long storage periods (Zhang et al., 2008) or during synthesis (Mandzy et al., 2005) or there could be preexisting aggregates that could not be dispersed by sonication (Saleh et al., 2005). However the 124-128 nm particle size range 
obtained in this study was sufficient to evaluate probe ultrasonication as an effective dispersion tool in breaking up nanoparticles and to investigate how the stability of IONPs changes in aquatic environment after being dispersed. Overall, with probe sonication as a dispersion tool, particles with hydrodynamic diameters between 117-147 $\mathrm{nm}$ remained in suspension over a 168 hour time period. Knowing that IONPs were still suspended, it was desirable to quantify the amount of IONPs that remain suspended in order to determine the stability of the IONPs dispersions.

\subsubsection{Stability of IONPs}

Nanoparticle stability can be defined as an even distribution of particles throughout the whole volume and the ability of the particles to stay separated from each other with time (Veronovski et al., 2010). However, it is also important that the particle size remain consistent for nanoparticle stability as aggregation to larger particles affects their mobility and reactivity. Although DLS has the ability to measure the hydrodynamic diameter of the particles that were currently suspended, it cannot quantify the amount of IONPs in the dispersion. Therefore, total iron analysis over a period of 168 hours was implemented as a means to assess the sedimentation of IONPs as well as to assist in the determination of the appropriate power level and sonication time to disperse IONPs into a stable suspension.

Stability experiments were performed on two concentrations of IONPs: 10 and $100 \mathrm{mg} / \mathrm{L}$. Figure 2.2 shows the percentage of IONPs dispersed over a course of 168 hours for a $10 \mathrm{mg} / \mathrm{L}$ IONPs dispersion at two power levels of sonication and at sonication times of 5, 20 and 60 minutes. The breakage of IONPs and the amount dispersed was strongly influenced by the energy input of the ultrasonic probe which is 

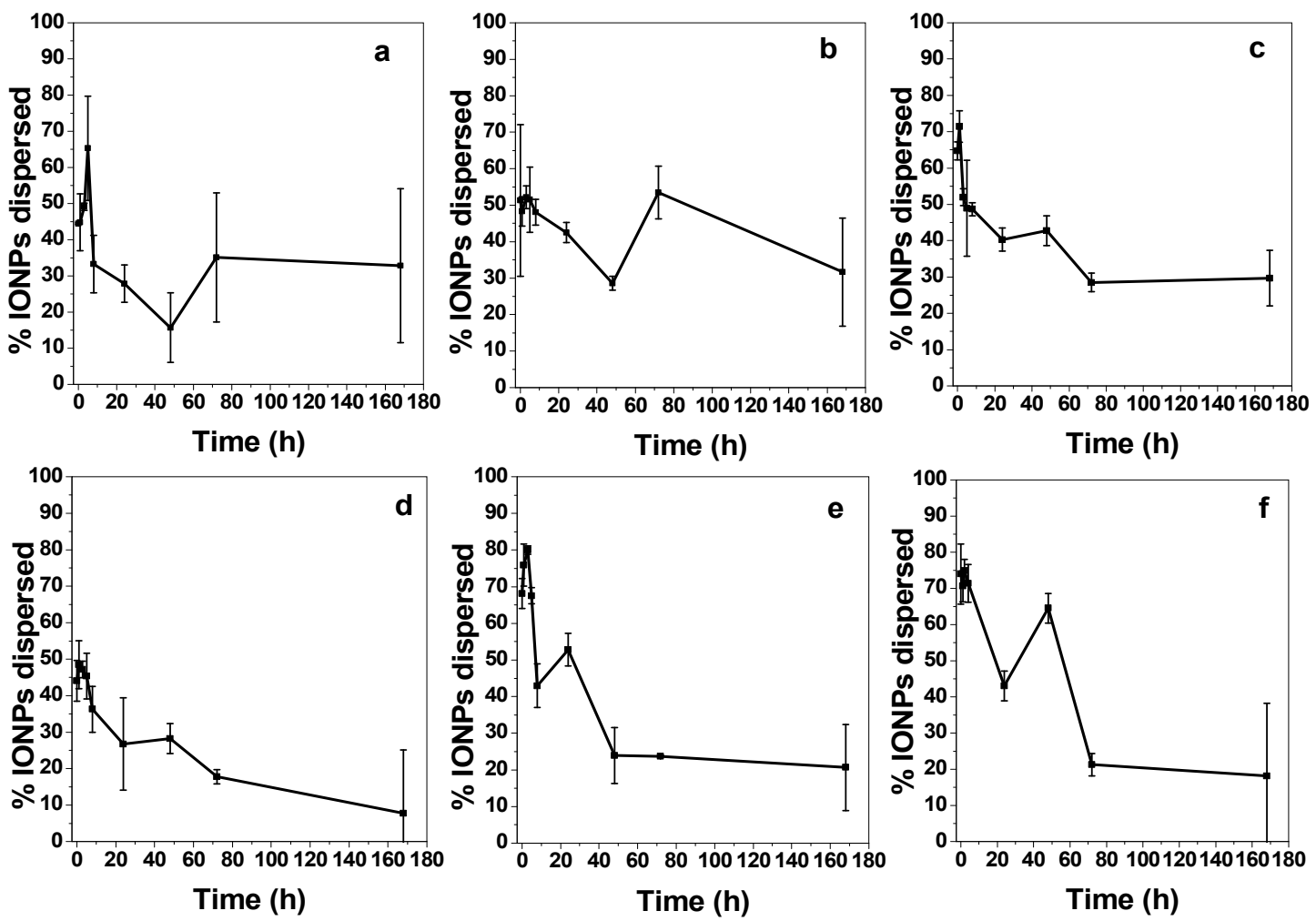

Figure 2. 2 The percentage of IONPs dispersed over a course of 168 hours for a $10 \mathrm{mg} / \mathrm{L}$ IONPs dispersion at two power levels of sonication and at sonication times of 5, 20 and 60 minutes. Graphs a, b and c represent dispersion for 5, 20 and 60 minutes of probe ultrasonication at power level 3. Graphs d, e and f represent dispersion for 5, 20 and 60 minutes of probe ultrasonication, respectively at power level 6 
controlled by the time, power and dispersion volume (Mandzy et al., 2005). In this experiment, the volume was constant for all samples so the time and power predominantly controlled the amount of IONPs dispersed. The greater the sonication power and the longer the sonication time, the greater the energy input and thus more efficient dispersion. Figures $2.2 \mathrm{a}, 2.2 \mathrm{~b}$ and $2.2 \mathrm{c}$ illustrate that an increase in time from 5 to 60 minutes for power level 3 increased the IONPs dispersion from 44 to $65 \%$ at $\mathrm{t}_{0}$. Similar results were observed for power level 6 as IONPs increased from 44 to $73 \%$ with increase in sonication time (Figures 2.2d, 2.2e and 2.2f). In addition an increase in power from level 3 (power density of $0.275 \mathrm{~W} / \mathrm{mL}$ ) to level 6 (power density of $0.55 \mathrm{~W} / \mathrm{mL}$ ) increased the amount of IONPs dispersed at $\mathrm{t}_{0}$ for sonication times of 20 and 60 minutes. However, equivalent amount ( $44 \%$ ) of IONPs was dispersed for 5 minutes of sonication regardless of the power level at $t_{0}$. The amount of IONPs dispersed increased from $51 \%$ to $86 \%$ at 20 minutes sonication and from $65 \%$ to $73 \%$ at 60 minutes when power increased from level 3 to level 6 . A similar trend was seen for the $100 \mathrm{mg} / \mathrm{L} \mathrm{IONPs}$ dispersion, in which the IONPs concentration in the suspension increases as sonication power and time increase (Figure 2.3). Monitoring the deposition over time (from $t_{0}$ to $t_{168}$ ) was important as it will provide information on how long these dispersions will remain stable overtime. As time progressed, aggregation occurred for both the 10 and $100 \mathrm{mg} / \mathrm{L}$ IONPs dispersions, as evidenced by the decrease in \% IONPs dispersed.

A comparison of the different sonication time at varying power levels indicates that sonicating for 20 minutes at power level 6 is the optimum dispersion condition. Sonicating for 60 minutes was time consuming and in most cases only slightly $(\sim 15 \%)$ 

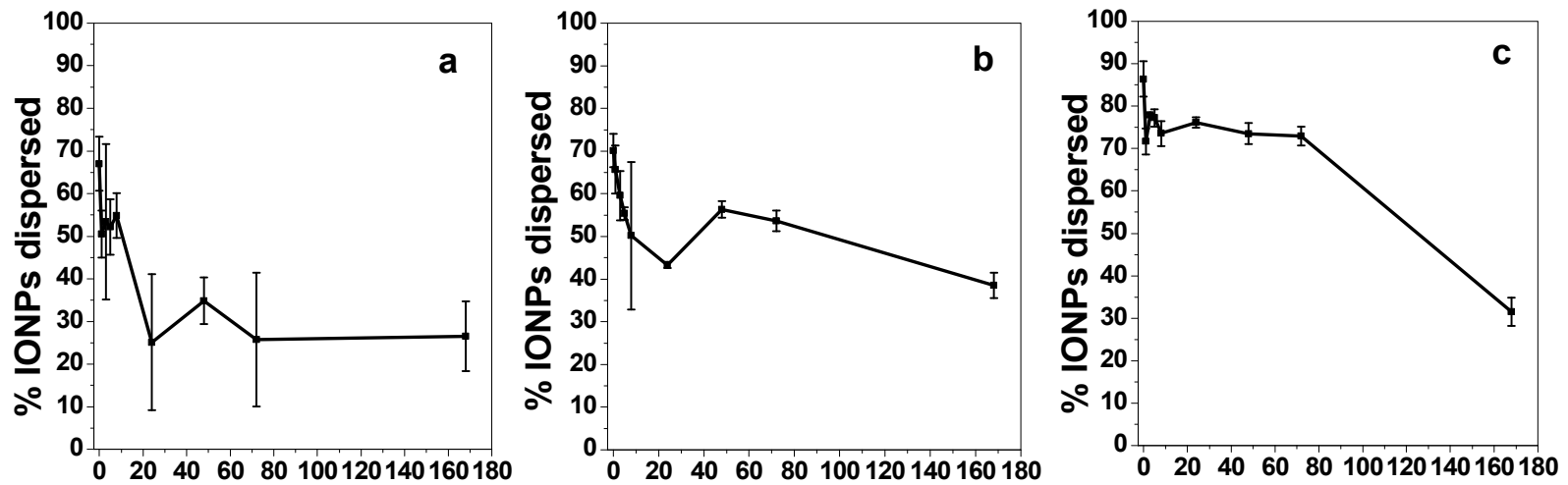

Time (h)

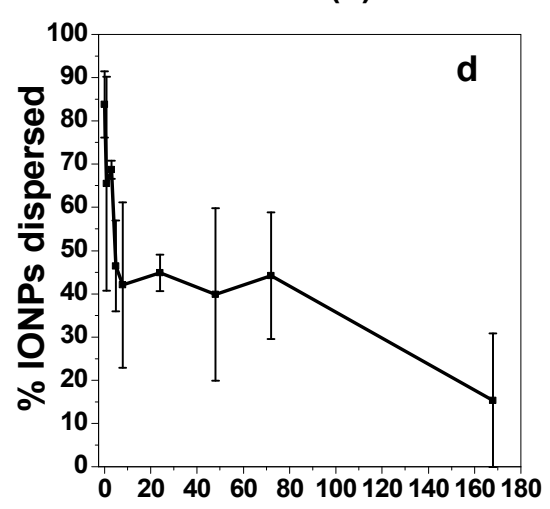

Time (h)

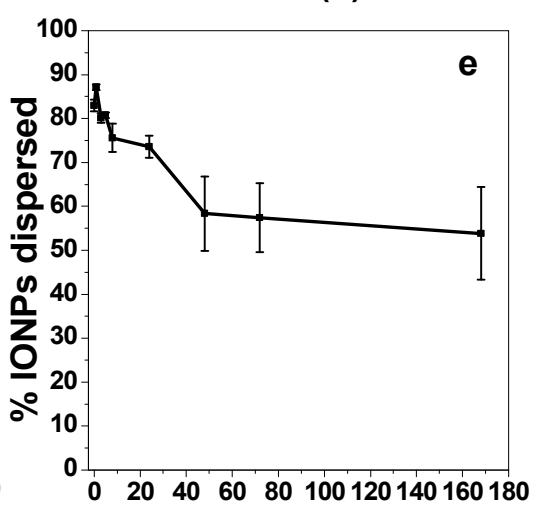

Time (h)

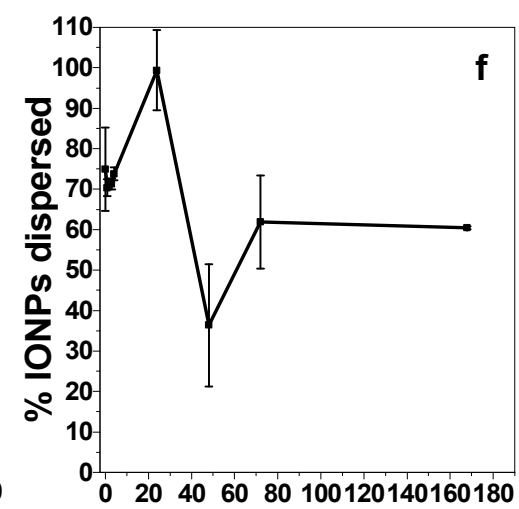

Time (h)

Figure 2. 3 The percentage of IONPs dispersed over a course of 168 hours for a $100 \mathrm{mg} / \mathrm{L}$ IONPs dispersed by ultrasonic probe.

Graphs a, b and c represent dispersion for 5, 20 and 60 minutes respectively, at power level 3. Graphs d, e and f represent dispersion for 5, 20 and 60 minutes, respectively at power level 6 . 
increased the amount of IONPs dispersed at $t_{0}$ when compared to 20 minutes. Sonicating for 5 minutes was not sufficient enough to disperse of the IONPs when compared to the other sonication times, and aggregation and sedimentation was faster compared to the other conditions.

The results of iron measurements show that a stable size distribution of IONPs in solution does not necessarily correlate with the concentration of IONPs. Sedimentation of IONPs is inevitable due to their tendency to aggregate resulted from the electrostatic, steric, and van der Waals forces (Jiang et al., 2009). As shown in this study, size measurements of IONPs at different times had consistent hydrodynamic diameters but particles still aggregate and sediment over time resulting in less and less IONPs being present in the suspensions. As often being overlooked, a stable size distribution does not imply that aggregation and sedimentation of particles does not occur. Monitoring both the particle size distribution and IONPs concentration over time can provide a better estimate on the stability of the dispersions.

\subsubsection{Effect of HA and ionic strength on the stability of IONPs}

\subsubsection{Effect of HA on IONPs Stability}

Humic acid is capable of adsorbing on iron oxides and thus can affect the surface charge, reactivity, and stability of the IONPs, which subsequently could influence the interaction of IONPs with metal contaminants (Mylon et al., 2004; Liu et al., 2010; Liu et al., 2011). Our study indicates that there was no significant change in the size of IONPs when HA was present. The hydrodynamic diameter ranged from $128-133 \mathrm{~nm}$ at $\mathrm{t}_{0}$ with the sizes slightly decreasing over the next 168 hour for all concentrations of IONPs (Figure 2.4). It was reported that the hydrodynamic radius between hematite colloids and 


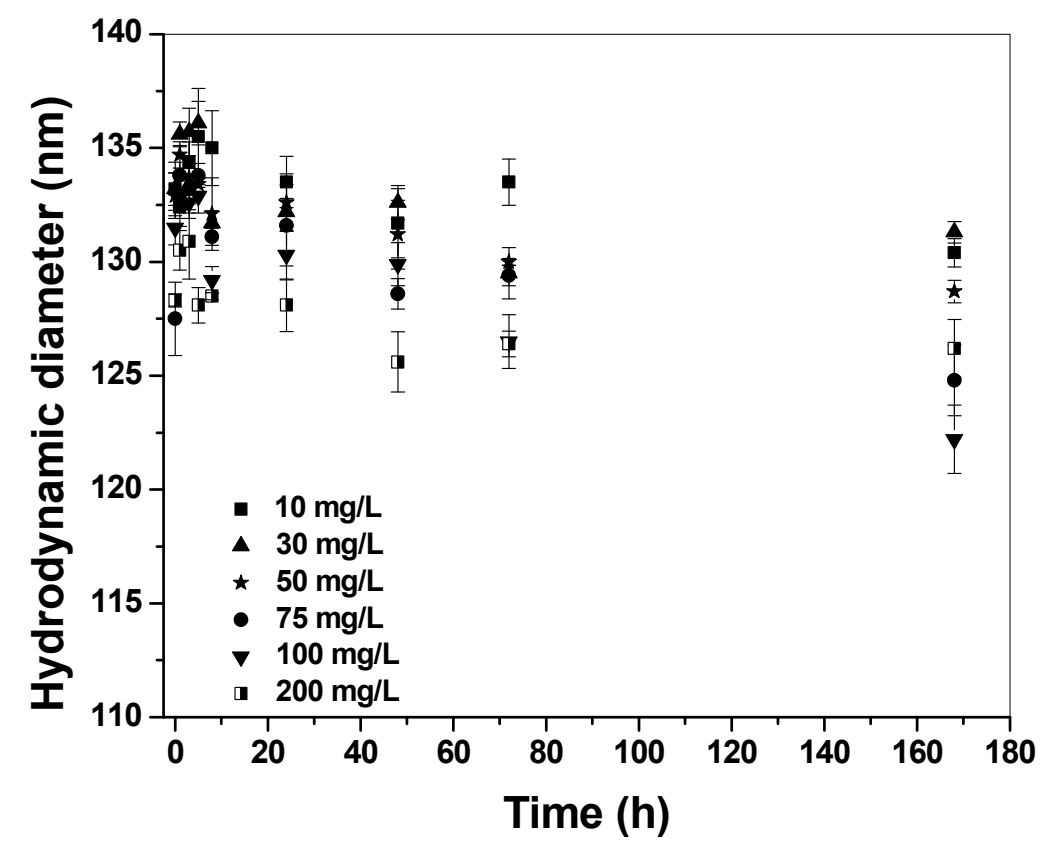

Figure 2. 4 The time dependent hydrodynamic diameter of 10, 30, 50, 75, 100 and 200 $\mathrm{mg} / \mathrm{L}$ IONPs dispersed by ultrasonic probe in the presence of humic acid $(100 \mathrm{mg} / \mathrm{L})$. All samples were dispersed using ultrasonic probe for 20 minutes at power level 6 .

natural organic matter (NOM) coated hematite was indistinguishable when using DLS measurements. It was reported that HA increased the layer thickness of small hematite colloids by only $<2 \mathrm{~nm}$ (Au et al., 1999; Mylon et al., 2004). Monitoring the IONPs concentration with time indicated that $70 \%$ or more IONPs were dispersed for all IONPs suspensions at $\mathrm{t}_{0}$ regardless of the nanoparticle concentration (dash lines in Figure 2.5). There was minimal sedimentation of IONPs from $t_{0}$ to $t_{168}$ with approximately $10-15 \%$ of IONPs sediment. The minimal sedimentation could be a result of the decrease in aggregation of the IONPs in the presence of HA resulting from the possibly coating of HA on the surface of the nanoparticles. It has been reported that HA can coat the surface 
of the nanoparticle thereby preventing aggregation through either electrostatic or steric interactions (Ghosh et al., 2010).
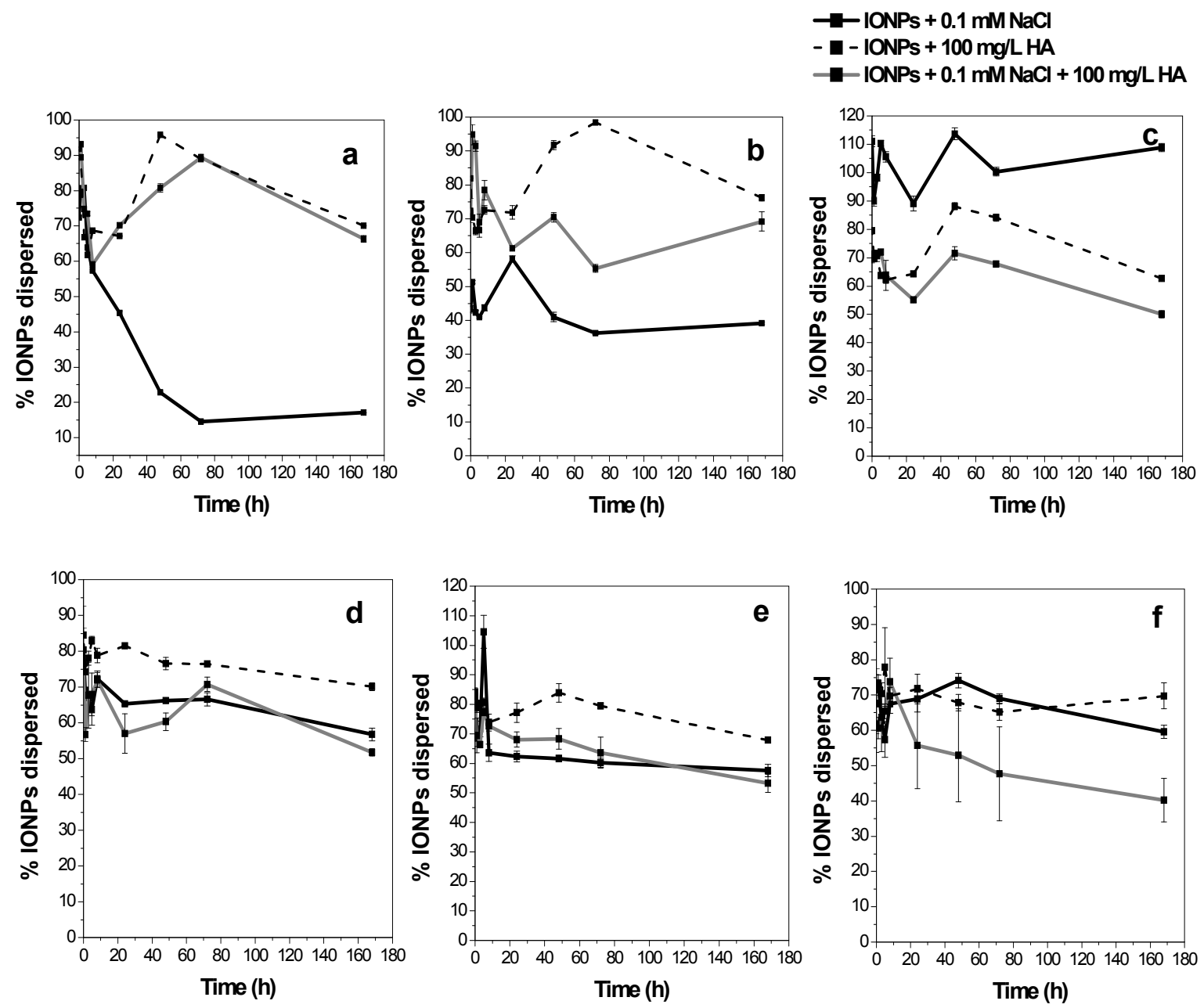

Figure 2. 5 The percentage of IONPs dispersed over a course of 168 hours while investigating the effect of $0.1 \mathrm{mM} \mathrm{NaCl}$ and $100 \mathrm{mg} / \mathrm{L} \mathrm{HA}$ (alone or in combination) on the dispersion of IONPs. Graphs a, b, c, d, e and f represent the percentage of IONPs dispersed in 10, 30, 50, 75, 100 and $200 \mathrm{mg} / \mathrm{L}$ IONPs suspension respectively. All samples were dispersed using ultrasonic probe for 20 minutes at power level 6 . 
Hematite nanoparticles have a point of zero charge (PZC) ranging from $\mathrm{pH} 5.5$ to 9.5 depending on the method of synthesis and experimental conditions (Schwertmann et al., 2000; He et al., 2008; Cerovic et al., 2009; Shipley et al., 2010).At this $\mathrm{pH}$ of 5.5 to 9.5, the repulsive force between IONPs decreases due to the lack of charge on the IONPs surface resulting in aggregation of the particles. Therefore the IONPs used in this study should experience a high degree of instability since all the samples in this study have measured $\mathrm{pH}$ ranging from $\mathrm{pH}$ 6.2-7.6, which is within the $\mathrm{PZC}$ range of hematite nanoparticles ( $\mathrm{pH}$ 5.5-9.5). Zeta potential measurements showed that the uncoated IONPs had a slight positive charge with zeta potential $+29.8 \pm 0.917 \mathrm{mV}$, while the HA coated IONPs was more negatively charged with a zeta potential of $-48.0 \pm 0.624 \mathrm{mV}$. HA has an abundant of carboxylic (-COOH, $\left.-\mathrm{COO}^{-}\right)$and phenolic $(-\mathrm{OH})$ functional groups that exist as negatively charged and it is believed that HA coating the surface can suppress any positive charge of the nanoparticle while enhancing the negative charge (Illes et al., 2006; Christian et al., 2008; Hu et al., 2010). Therefore, the minimal sedimentation of IONPs in the presence of HA (Figure 2.5, broken lines) compared to that without HA (Figures 2.2 and 2.3) could be explained by HA coating the IONPs surface leading to a change in the surface charge of the IONPs from a slightly positive charge to a stronger negative charge. The stronger negative charge with HA present possibly has a greater repulsive force compared to the slightly positive charge of IONPs without HA, thus enhancing stability through electrostatic interactions (Ghosh et al., 2010).

\subsubsection{Effect of ionic strength on IONPs Stability}

The DLVO theory can be used to explain the stability of IONPs dispersions. The van der Waals attractive forces and electrical double layer repulsive force between 
particles plays a significant role in controlling the aggregation of particles. The ionic strength has a strong influence on the thickness of the electrical double layer. An increase in ionic strength would lead to a decrease in the electric double layer thickness resulting from the compression of the electric double-layer causing aggregation of particles (Zhang et al., 2008; Jiang et al., 2009). To examine the effect of ionic strengths on IONPs aggregation, several concentrations of $\mathrm{NaCl}(0.1,10$ and $100 \mathrm{mM})$ were tested.

At low ionic strength $(0.1 \mathrm{mM})$, the hydrodynamic diameter ranged from $115-130$ nm (Figure 2.6) over the course of 168 hours.

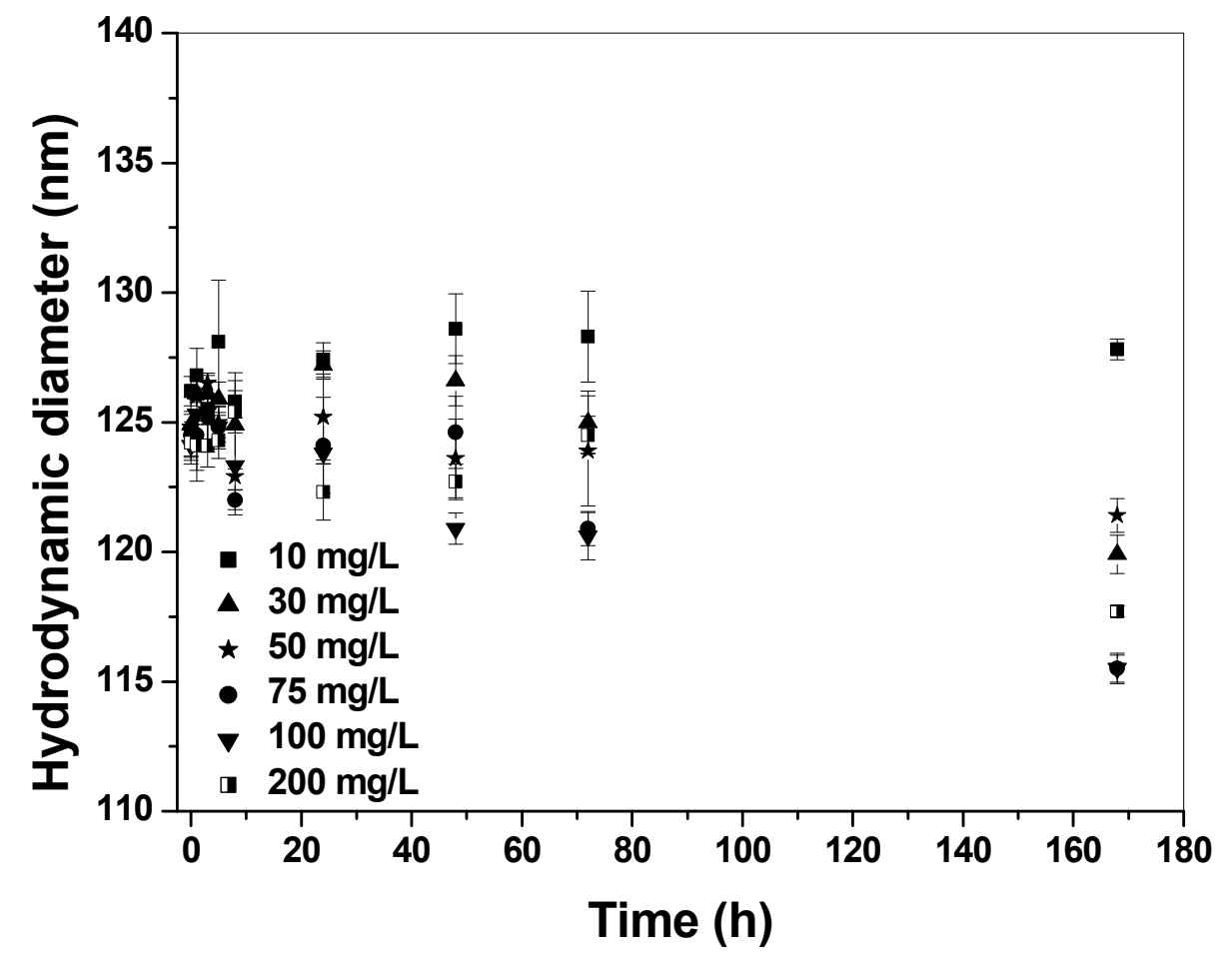

Figure 2. 6 The time dependent hydrodynamic diameter of 10, 30, 50, 75, 100 and 200 $\mathrm{mg} / \mathrm{L}$ IONPs dispersed by ultrasonic probe in the presence of $\mathrm{NaCl}(0.1 \mathrm{mM})$. All samples were dispersed using ultrasonic probe for 20 minutes at power level 6 . 
These results were not significantly different from the size of IONPs alone (Figure 2.1b), indicating that at low ionic strength, the electrostatic repulsive force is dominant over the attractive force therefore the particle size was not altered.

In regards to the quantity of IONPs suspended over time, Figure 2.5 (dark solid lines) shows that approximately $50 \%$ or more IONPs was dispersed for the $10,30,50,75$, 100 and $200 \mathrm{mg} / \mathrm{L}$ dispersions. Majority of the IONPs had remained dispersed with only $10-15 \%$ deposition of IONPs from $t_{0}$ to $t_{168}$ for all IONPs concentration except for the 10 $\mathrm{mg} / \mathrm{L}$ dispersion (Figure $2.5 \mathrm{a}$, dark solid line). The $10 \mathrm{mg} / \mathrm{L}$ dispersion had the greatest sedimentation over time and this could possibly be a result of a concentration effect (see below). Similarly to the addition of $0.1 \mathrm{mM} \mathrm{NaCl}$ to the nanoparticle suspension, the addition of $10 \mathrm{mM} \mathrm{NaCl}$ resulted in aggregation and sedimentation that was depended upon the nanoparticle concentrations (data not shown). An increase in ionic strength to 10 $\mathrm{mM} \mathrm{NaCl}$ resulted in rapid aggregation and sedimentation for the $10,30,50$ and $75 \mathrm{mg} / \mathrm{L}$ IONPs dispersion within 8 hours as evidenced by a deposit of nanoparticles at the bottom of sample containers as well as unsuccessful DLS measurements due to the intensity of the scattered light not satisfactory for quantitative determination of particle size. However, the 100 and $200 \mathrm{mg} / \mathrm{L}$ suspensions were not significantly affected and were stable for DLS measurements. Hydrodynamic diameters for the $100 \mathrm{mg} / \mathrm{L}$ dispersion ranged from $128 \mathrm{~nm}$ at $\mathrm{t}_{0}$, increasing to $174 \mathrm{~nm}$ at $\mathrm{t}_{72}$ then slightly decreased in size to $150 \mathrm{~nm}$ at $\mathrm{t}_{168}$ (Figure 2.7). On the other hand, the $200 \mathrm{mg} / \mathrm{L}$ dispersion had minimal changes in size $(110-120 \mathrm{~nm})$. This difference could possibly be accounted for by the compression of the electric double layer being more feasible in low IONPs concentration as a result of the ionic strength being high enough to cause aggregation of the nanoparticles. 


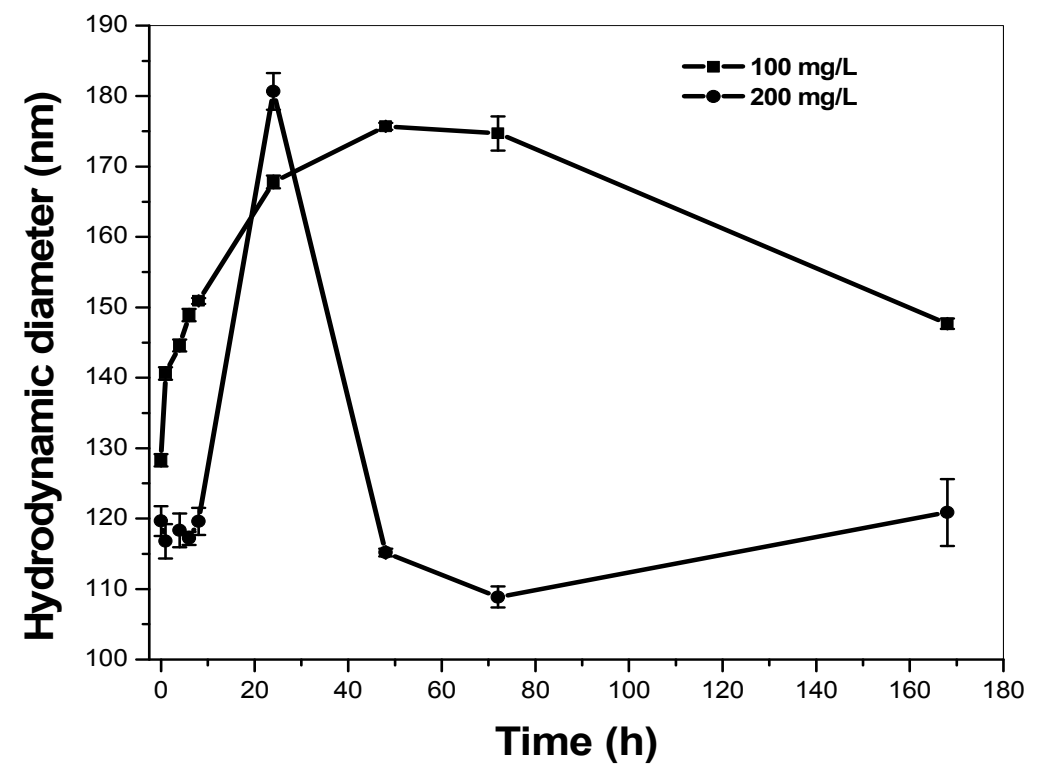

Figure 2. 7 The time dependent hydrodynamic diameter of 100 and $200 \mathrm{mg} / \mathrm{L}$ IONPs dispersions in the presence of $10 \mathrm{mM} \mathrm{NaCl}$. Samples were dispersed using ultrasonic probe for 20 minutes at power level 6 .

A further increase in ionic strength $(100 \mathrm{mM})$ caused rapid aggregation and sedimentation of all the IONPs dispersions within 4 hours. Similarly to $10 \mathrm{mM}$ ionic strength, deposits of nanoparticles accumulated at the bottom of sample containers and DLS measurements were unsuccessful due to the polydispersity of the suspensions. At this ionic strength, van der Waals attractive forces dominate over the repulsive forces causing significant aggregation. In addition, metal oxides have large Hamaker constants and electrostatic stabilization is usually achieved at low ionic strength.

\subsubsection{Stability of IONPs in the presence of both HA and low ionic strength}

The aggregation behavior of IONPs was investigated in the coexistence of low ionic strength $(0.1 \mathrm{mM} \mathrm{NaCl})$ and $\mathrm{HA}$. Figure 2.8 shows that the hydrodynamic 
diameters ranged from 132 to $135 \mathrm{~nm}$ at $\mathrm{t}_{0}$ with sizes decreasing slightly over the course of 168 hours. These sizes are similar to the hydrodynamic diameter of IONPs in the presence of HA alone (128-133 nm, Figure 2.4) and in the presence of low ionic strength alone (115-130 nm, Figure 2.6).

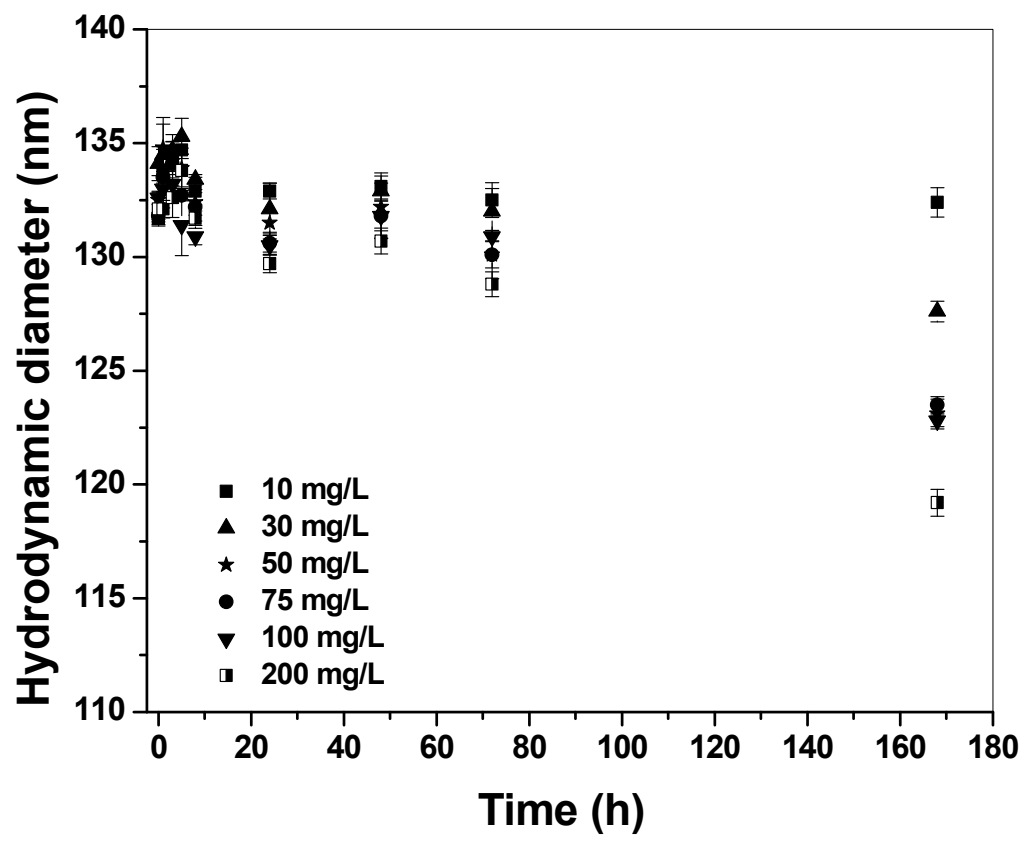

Figure 2. 8 The time dependent hydrodynamic diameter of 10, 30, 50, 75, 100 and 200 $\mathrm{mg} / \mathrm{L} \mathrm{IONPs} \mathrm{dispersed} \mathrm{by} \mathrm{ultrasonic} \mathrm{probe} \mathrm{in} \mathrm{the} \mathrm{presence} \mathrm{of} \mathrm{HA}(100 \mathrm{mg} / \mathrm{L})$ and $\mathrm{NaCl}$ $(0.1 \mathrm{mM})$. All samples were dispersed using ultrasonic probe for 20 minutes at power level 6.

The amount of nanoparticles dispersed was $65 \%$ or more at $\mathrm{t}_{0}$ with minimal sedimentation for all IONPs over 168 hours (Figure 2.5, light solid lines). The large sedimentation of IONPs that was seen for the $10 \mathrm{mg} / \mathrm{L}$ dispersion in the presence of 
$0.1 \mathrm{mM} \mathrm{NaCl}$ alone, was not observed when both $\mathrm{HA}$ and $\mathrm{NaCl}$ were present. The instability caused by the ionic strength could have been overcome by the stabilization effect of HA. Overall, the minimal sedimentation could be a result of HA coating the surface of the nanoparticles causing both steric and electrostatic stabilization as well as the ionic strength being low enough to allow for electrostatic repulsive force to be dominant therefore enhancing the stability of IONPs. Therefore, IONPs dispersions can be prepared under different environmental factors with these dispersions being stable for a prolonged period of time.

\subsection{Conclusions}

In this study, we determined that probe ultrasonication is an appropriate tool to disperse IONPs. Although probe ultrasonication did not break the IONPs down into the primary particle size, this method had proven to be more effective in dispersing IONPs compared to dispersion via vortex or bath sonication. It is important to be aware that a stable size distribution of IONPs in solution does not necessarily correlate with the concentration of IONPs therefore it is necessary to monitor the concentration of IONPs in stability studies. An increase in ultrasonication time and power increased the amount of IONPs dispersed. Although sedimentation occurred to some extent, a considerable amount of IONPs remained in suspension in the presence and absence of $100 \mathrm{mg} / \mathrm{L} \mathrm{HA}$ and $0.1 \mathrm{mM} \mathrm{NaCl}$. Results showed that high ionic strengths increased colloidal instability by compressing the electrical double layer thickness causing rapid aggregation and sedimentation. On the other hand, the electrostatic repulsive force dominated at low ionic strengths resulting in reduced destabilization of the dispersions. The addition of modifiers to enhance stability of IONPs may not be necessary as the presence of HA, which is 
naturally found in the environment, could enhance the colloidal stability of IONPs through possible steric and electrostatic repulsion. These results are important as stable IONPs dispersions can be prepared without the use of synthetic modifiers. This research provides us with a useful tool for development of a laboratory scale methodology to study and understand the adsorption mechanisms of toxic environmental contaminants with unmodified IONPs.

\subsection{List of References}

Baalousha M. Aggregation and disaggregation of iron oxide nanoparticles: Influence of particle concentration, $\mathrm{pH}$ and natural organic matter. Sci Total Environ 2009; 407: 2093-2101.

Baalousha M, Manciulea A, Cumberland S, Kendall K, Lead JR. Aggregation and surface properties of iron oxide nanoparticles: influence of $\mathrm{pH}$ and natural organic matter. Environ Toxicol Chem 2008; 27: 1875-1882.

Cerovic L, Lefevre G, Jaubertie A, Fedoroff M, Milonjic S. Deposition of hematite particles on polypropylene walls in dynamic conditions. J Colloid Interface Sci 2009; 330: 284-291.

Christian P, Von der Kammer F, Baalousha M, Hofmann T. Nanoparticles: structure, properties, preparation and behavior in environmental media. Ecotoxicology 2008; 17: 326-343.

Deryagin B, Landau L. Theory of the stability of strongly charged lyophobic sols and of the adhesion of strongly charged particles in solutions of electrolytes. Acta Physicochim 1941; 14: 633-62.

Ghosh S, Mashayekhi H, Bhowmik P, Xing B. Colloidal Stability of Al2O3 Nanoparticles as Affected by Coating of Structurally Different Humic Acids. Langmuir 2010; 26: 873-879.

He F, Zhao D. Manipulating the Size and Dispersibility of Zerovalent Iron Nanoparticles by Use of Carboxymethyl Cellulose Stabilizers. Environ Sci Technol 2007; 41: 6216-6221.

He YT, Wan J, Tokunaga T. Kinetic stability of hematite nanoparticles: the effect of particle sizes. J Nanopart Res 2008; 10: 321-332. 
Hu J-D, Zevi Y, Kou X-M, Xiao J, Wang X-J, Jin Y. Effect of dissolved organic matter on the stability of magnetite nanoparticles under different $\mathrm{pH}$ and ionic strength conditions. Sci Total Environ 2010; 408: 3477-3489.

Illes E, Tombacz E. The effect of humic acid adsorption on $\mathrm{pH}$-dependent surface charging and aggregation of magnetite nanoparticles. J Colloid Interface Sci 2006; 295: 115-123.

Jiang J, Oberdorster G, Biswas P. Characterization of size, surface charge, and agglomeration state of nanoparticle dispersions for toxicological studies. J Nanopart Res 2009; 11: 77-89.

Kanel SR, Nepal D, Manning B, Choi H. Transport of surface-modified iron nanoparticle in porous media and application to arsenic(III) remediation. J Nanopart Res 2007; 9: 725-735.

Kim H-J, Phenrat T, Tilton RD, Lowry GV. Fe0 Nanoparticles Remain Mobile in Porous Media after Aging Due to Slow Desorption of Polymeric Surface Modifiers. Environ Sci Technol 2009; 43: 3824-3830.

Liu G, Cai Y. Complexation of arsenite with dissolved organic matter: Conditional distribution coefficients and apparent stability constants. Chemosphere 2010;81: 890-896.

Liu G, Fernandez A, Cai Y. Complexation of Arsenite with Humic Acid in the Presence of Ferric Iron. Environ Sci Technol 2011; 45: 3210-3216.

Mandzy N, Grulke E, Druffel T. Breakage of TiO2 agglomerates in electrostatically stabilized aqueous dispersions. Powder Technol 2005; 160: 121-126.

Mylon SE, Chen KL, Elimelech M. Influence of Natural Organic Matter and Ionic Composition on the Kinetics and Structure of Hematite Colloid Aggregation: Implications to Iron Depletion in Estuaries. Langmuir 2004; 20: 9000-9006.

Petosa AR, Jaisi DP, Quevedo IR, Elimelech M, Tufenkji N. Aggregation and Deposition of Engineered Nanomaterials in Aquatic Environments: Role of Physicochemical Interactions. Environ Sci Technol 2010; 44: 6532-6549.

Phenrat T, Liu Y, Tilton RD, Lowry GV. Adsorbed Polyelectrolyte Coatings Decrease Fe Nanoparticle Reactivity with TCE in Water: Conceptual Model and Mechanisms. Environ Sci Technol 2009; 43: 1507-1514.

Phenrat T, Saleh N, Sirk K, Tilton RD, Lowry GV. Aggregation and Sedimentation of Aqueous Nanoscale Zerovalent Iron Dispersions. Environ Sci Technol 2007; 41: 284-290. 
Saleh N, Phenrat T, Sirk K, Dufour B, Ok J, Sarbu T, et al. Adsorbed triblock copolymers deliver reactive iron nanoparticles to the oil/water interface. Nano Lett 2005; 5: 2489-2494.

Santos HM, Capelo JL. Trends in ultrasonic-based equipment for analytical sample treatment. Talanta 2007; 73: 795-802.

Schrick B, Hydutsky BW, Blough JL, Mallouk TE. Delivery Vehicles for Zerovalent Metal Nanoparticles in Soil and Groundwater. Chem Mater 2004; 16: 2187-2193.

Schwertmann U, Cornell RM. Iron Oxides in the Laboratory: Preparation and Characterization. Weinheim: Wiley, 2000.

Shipley H, Engates K, Guettner A. Study of iron oxide nanoparticles in soil for remediation of arsenic. J Nanopart Res 2010: 1-11-11.

Sun Y-P, Li X-q, Cao J, Zhang W-x, Wang HP. Characterization of zero-valent iron nanoparticles. Adv Colloid Interface Sci 2006; 120: 47-56.

Sun Y-P, Li X-Q, Zhang W-X, Wang HP. A method for the preparation of stable dispersion of zero-valent iron nanoparticles. Colloids Surf A 2007; 308: 60-66.

Suslick KS, Price GJ. Applications of ultrasound to materials chemistry. Annu Rev Mater Sci 1999; 29: 295-326.

Theron J, Walker JA, Cloete TE. Nanotechnology and water treatment: Applications and emerging opportunities. Crit Rev Microbiol 2008; 34: 43-69.

Tiraferri A, Chen KL, Sethi R, Elimelech M. Reduced aggregation and sedimentation of zero-valent iron nanoparticles in the presence of guar gum. J Colloid Interface Sci 2008; 324: 71-79.

Veronovski N, Andreozzi P, La Mesa C, Sfiligoj-Smole M. Stable TiO2 dispersions for nanocoating preparation. Surf Coat Technol 2010; 204: 1445-1451.

Verwey EJW. Theory of the stability of lyophobic colloids. J Phys Colloid Chem 1947; 51: 631-6.

Verwey EJW, Overbeek JTG. Theory of the Stability of Lyophobic Colloids. Elsevier: Amsterdam, 1948.

Wang C-B, Zhang W-x. Synthesizing Nanoscale Iron Particles for Rapid and Complete Dechlorination of TCE and PCBs. Environ Sci Technol 1997; 31: 2154-2156.

Yang GCC, Tu H-C, Hung C-H. Stability of nanoiron slurries and their transport in the subsurface environment. Sep Purif Technol 2007; 58: 166-172. 
Zhang Y, Chen Y, Westerhoff P, Hristovski K, Crittenden JC. Stability of commercial metal oxide nanoparticles in water. Water Res 2008; 42: 2204-2212. 


\section{Chapter III}

Interactions of arsenite and arsenate with hematite nanoparticles and aggregates: sorption kinetics and isotherm studies. 


\subsection{Abstract}

The mobility and bioavailability of arsenic is strongly influenced by the adsorption onto iron oxide surfaces. In the environment, arsenic is known to adsorb onto bulk zerovalent iron (ZVI), iron oxides and oxy-hydroxides but this process can be enhanced using nanoparticulate iron oxides. These nanoparticles have increased reactivity and remediation capability compared to their bulk sizes. Kinetic and thermodynamic parameters are lacking for the comparative adsorption of arsenite and arsenate to bare hematite particles and how the particle size influences the adsorption process. Therefore, the objective of the present study was to compare the adsorption kinetics and adsorption isotherm of arsenite and arsenate on bare hematite nanoparticles and aggregates. Kinetic data were best described by a pseudo second-order model. As(V) and As(III) had similar rate constants as rapid adsorption occurred within the first $8 \mathrm{~h}$ regardless of particle size. However, hematite nanoparticles and aggregates showed a higher affinity to adsorb larger amounts of $\mathrm{As}(\mathrm{V})$ than $\mathrm{As}(\mathrm{III})$ at equilibrium. We were able to show that aggregation and sedimentation of hematite nanoparticles occurs during the adsorption process especially during the period of rapid arsenic adsorption. Isotherm studies were described by the Freundlich model and it confirmed that hematite nanoparticles has a significantly higher adsorption capacity for both $\mathrm{As}(\mathrm{V})$ and $\mathrm{As}(\mathrm{III})$ than hematite aggregates. The information gathered is useful and can assist in predicting arsenic adsorption behavior and assessing the role of iron oxide nanoparticles in the biogeochemical cycling of arsenic. 


\subsection{Introduction}

Iron is the fourth most abundant element in the earth's crust and reactions involving iron can play a significant role in the cycling of metals (Schwertmann et al., 2000; Cundy et al., 2008). Iron oxides are widespread in nature and usually occur as a result of the oxidization of $\mathrm{Fe}(0)$ when exposed to oxygen in water or air (Stipp et al., 2002; Zhang, 2003). Sorption of contaminants onto bulk zerovalent iron (ZVI), iron oxides and oxy-hydroxides is known to occur widely in the environment (Zaspalis et al., 2007). Several studies have investigated the adsorption of metals to bulk iron and iron oxides (Jain et al., 1999; Jeong et al., 2007; Kundu et al., 2007; Banerjee et al., 2008). The process can be enhanced using zerovalent iron nanoparticles (NZVI) and iron oxide nanoparticles (IONPs) (Dixit et al., 2003). The application of NZVI and IONPs for environmental cleanup has gained interest as their unique physical and chemical properties allow for remediation of chlorinated compounds and heavy metals from aquatic environment (Wang et al., 1997; He et al., 2007; Phenrat et al., 2007; Shipley et al., 2010a). The strong adsorption characteristics for metals to IONPs are important as this can control the fate and bioavailability of heavy metals in the environment.

Arsenic is naturally present in groundwater and is commonly introduced into the environment through both natural formation (weathering, biological activity, and volcanic activity) and anthropogenic activity (mining, smelting, and direct use of arseniccontaining herbicides by industry and agriculture) (Mandal et al., 2002; Zhang et al., 2002). The human population can become exposed to arsenic through numerous ways but the most significant one is through ingestion of arsenic in drinking water or food. The environmental impact of arsenic contamination is a global problem because of its high 
level of toxicity. Arsenic is a well-known carcinogen and has been linked to cancer of the bladder, lungs, skin, kidney, nasal passages, liver, and prostate (Ng et al., 2003; Shipley et al., 2009). The severe effects of long term exposure to arsenic resulted in an EPA reduction of arsenic standard in drinking water from 50 to $10 \mu \mathrm{g} / \mathrm{L}$. Arsenic in the natural waters is predominantly in the inorganic forms: pentavalent arsenate $(A s(V))$ or the trivalent arsenite (As(III)) (Ko et al., 2004; Zaspalis et al., 2007). However, the degree of toxicity is dependent upon the oxidation state with the reduced form arsenite being more toxic and mobile than arsenate (Kundu et al., 2007; Carabante et al., 2009).

The reactivity of arsenic in the environment can be in the form of adsorption, ligand exchange, oxidation-reduction, and precipitation (Banerjee et al., 2008). However, adsorption to metal oxides particularly iron oxides to form inner-sphere complexes is one of the most significant factor controlling arsenic mobility in the environment (Al-Abed et al., 2006). Iron oxides in general, whether in the bulk form or nanoparticulate form, have strong binding affinity for both arsenite and arsenate. The application of iron oxide nanoparticles (IONPs) in contaminant remediation has enhanced the adsorption process due to the large surface area and its strong adsorption characteristics. These nanoparticles have increased adsorption capacity while maintaining many of the properties of bulk iron oxides. Numerous studies have investigated the adsorption behavior of arsenic with bulk iron oxides (Raven et al., 1998; Jain et al., 1999; Zhang et al., 2004; Jeong et al., 2007; Banerjee et al., 2008), but only a few studies have investigated arsenic adsorption behavior with bare/unmodified iron oxide nanoparticles (Auffan et al., 2008; Tuutijarvi et al., 2009; Shipley et al., 2010b; Turk et al., 2010). At environmental pH, iron oxides nanoparticle suspensions are highly unstable and aggregation occurs. In recent years, the 
new trend is the application of stabilizers/modifiers to prevent aggregation. Stabilizers or modifiers are soluble polymers or surfactants that are attached to the nanoparticles, providing strong interparticle electrostatic and steric repulsion that will overcome the van der waals attraction and magnetic forces (He et al., 2007). Although this enhances nanoparticle stability, modifiers could affect the sorption and desorption of contaminants on the nanoparticle surface, introduce additional mass transfer limitations of contaminants to active surface sites, and could decrease the reaction rate of the reactions that could be taking place at the surface (Phenrat et al., 2009). Therefore, it is necessary to study the interactions of bare/ unmodified iron oxide nanoparticles with arsenic so as to understand the true interactions of nanoparticles with arsenic in the environment. .

As a consequence of the prevalence and toxicity of arsenic, its strong binding affinity for iron oxides surface and the development of nanotechnology as a potential remediation tool for toxic metals contaminants in the environment, it is of great interest that we evaluate the effects of IONPs on the fate and mobility of arsenate and arsenite in aqueous environment. The adsorption of arsenic and its retention on IONPs should be investigated while considering several factors such as the aggregation of the nanoparticles and the type of sorbed species. Studies in the literature focusing on arsenic adsorption behavior with IONPs had failed to monitor IONPs particle size during adsorption. From our previous studies (Dickson et al., 2012) and that of Zhang et al. 2008, IONPs tend to aggregate either at the initial stage of experiments and as time persist. These IONPS can grow to micron size particles during the adsorption process and will eventually sediment. The IONPs sedimentation will influence the adsorptive properties of IONPs and its interaction with arsenic. Hence, previous arsenic studies in the literature 
might not be representative of nanoparticle behavior. The potential aggregation of the nanoparticles can retard adsorption by reducing the surface area consequently decreasing IONPs reactivity and reducing the efficiency of arsenic adsorption (He et al., 2008). However, aggregation can be beneficial in the immobilization of arsenic by trapping the arsenic in the interior portions of the iron oxide aggregates thus reducing its bioavailability (Waychunas et al., 2005). Therefore, it is important to understand and demonstrate the difference of arsenic adsorption between nanosized iron oxides and aggregated iron oxides. It is also important to investigate the adsorption of different arsenic species with IONPs and IONPs aggregates since the degree of toxicity and mobility is dependent upon the oxidation state.

In the present study, the adsorption/desorption process between nanoparticulate iron oxides and iron oxides aggregates was investigated with unmodified hematite $(\alpha-$ $\mathrm{Fe}_{2} \mathrm{O}_{3}$ ). Hematite is one of the most thermodynamically stable iron oxides (Schwertmann et al., 2000). It is usually the final stage of iron oxide phase transformations where unstable phases such as ferrihydrite and lepidocrocite are transformed into more stable phases such as goethite and hematite (Pedersen et al., 2006). Phase transformation can be detrimental to adsorption since adsorbates can be desorbed and become bioavailable during the process. Therefore hematite has a greater potential for contaminant adsorption due to its stability. These hematite nanoparticles will have no surface modifications as modifiers could affect the sorption and desorption of contaminants on the nanoparticle surface, introduce additional mass transfer limitations of contaminants to active surface sites, and could decrease the reaction rate of the reactions that could be taking place at the surface (Phenrat et al., 2009). Therefore, we investigated and compared the adsorption 
process of $\mathrm{As}(\mathrm{V})$ and $\mathrm{As}(\mathrm{III})$ with hematite nanoparticles and hematite aggregates. The probe ultrasonication dispersion method was utilized to produce nanosized iron oxides from commercially bought nanoparticle powder and a conventional method was used to mimic IONPs that may be aggregated at the early stage of experiments. The goal was to understand the kinetics and thermodynamics of arsenic-IONPs interactions. In doing so, I determined kinetic parameters, adsorption isotherms and adsorption capacities, thus evaluating the role of hematite nanoparticles and aggregates in the fate, mobility and bioavailability of arsenic in the environment.

\subsection{Procedures and Methods}

\subsubsection{Materials and Chemicals}

Commercial iron oxide nanoparticles $\left(\alpha-\mathrm{Fe}_{2} \mathrm{O}_{3}, 98 \%\right.$ purity and $50 \mathrm{~m}^{2} / \mathrm{g}$ specific surface area) were purchased from Nanostructured and Amorphous Materials (Houston, Texas). Stock solutions of $1000 \mathrm{mg} / \mathrm{L} \mathrm{As} \mathrm{(III)} \mathrm{and} \mathrm{As} \mathrm{(V)} \mathrm{were} \mathrm{prepared} \mathrm{by} \mathrm{dissolving}$ sodium (meta)arsenite $\left(\mathrm{NaAsO}_{2}, 98 \%\right.$ purity) and sodium arsenate dibasic heptahydrate $\left(\mathrm{Na}_{2} \mathrm{HAsO}_{4} \cdot 7 \mathrm{H}_{2} \mathrm{O}, 99 \%\right.$ purity), respectively in nanopure $18.2 \mathrm{M} \Omega$ water produced from a nanopure diamond lab water system (Barnstead Thermolyne Corporation, Dubuque, IA). The arsenic standards were reagent grade and obtained from Aldrich Chemical Company (Milwaukee, WI.).

\subsubsection{Instrumentation}

Iron oxide nanoparticles were dispersed by probe ultrasonication using a Fisher Scientific sonic dismembrator model 100 (Pittsburg, PA) to prepare nanosize hematite particle. The IONPs were dispersed by vortex using a Fisher Scientific touch mixer model 232 (Pittsburgh, PA) to prepare micrometer size particles. Dynamic Light 
Scattering (DLS), a Malvern Zetasizer Nano-ZS (Westborough, MA.), was employed for particle size monitoring and zeta potential measurements. Field emission scanning electron microscopy (FE-SEM, JEOL JSM-6330F) was used for particle size measurement and morphology. Total iron concentration was monitored using a graphite furnace atomic absorption spectrometer (GFAAS) (Perkin Elmer, model Aanalyst 600). The $\mathrm{pH}$ measurements were made using a Fisher Scientific accumet Research AR15 $\mathrm{pH} / \mathrm{mV} /{ }^{\circ} \mathrm{C}$ Meter. Quantitative determination of arsenic concentration was conducted using Inductively Coupled Plasma-Mass Spectrometry (ICP-MS) (Perkin Elmer). Prior to arsenic analysis by ICP-MS, samples were centrifuged with a Fisher Marathon 21000R centrifuge (Needham Heights, MA).

\subsubsection{Experimental Procedures}

\subsubsection{Characterization of Hematite Nanoparticles and Aggregates}

The $10 \mathrm{mg} / \mathrm{L}$ hematite dispersions (prepared by probe ultrasonication and vortex) were filtered through $0.1 \mu \mathrm{m}$ membrane filter made of cellulose of esters material (Millipore). The filters were dried overnight and the particles were coated with gold using an auto sputter coater (Pelco SC-7) before FE-SEM imaging. The particle size and morphology was obtained at $15 \mathrm{kV}$ using the secondary electron imaging mode. DLS was also used to determine the particle size of the hematite dispersions.

\subsubsection{Adsorption Kinetics Experiments}

Adsorption studies were performed by mixing $10 \mathrm{mg} / \mathrm{L}$ hematite nanoparticles with $200 \mu \mathrm{g} / \mathrm{L}$ of $\mathrm{As}(\mathrm{V})$ or $\mathrm{As}(\mathrm{III})$ in $50 \mathrm{~mL}$ polypropylene centrifuge tubes. Firstly, IONPs were dispersed using probe ultrasonication for 20 minutes at power level 6 to generate nanosize hematite particles. In this study, iron oxides that are dispersed using 
probe ultrasonication are referred to as hematite nanoparticles. After dispersion, the suspension was spiked with $200 \mu \mathrm{g} / \mathrm{L}$ of arsenic standard $(10 \mathrm{mg} / \mathrm{L})$. All samples were prepared in nanopure $18.2 \mathrm{M} \Omega$ water and the $\mathrm{pH}$ ranged from $6.8-8$ as the suspensions were not buffered. The experiment was performed at room temperature $\left(20^{\circ} \mathrm{C}\right)$ and the samples were agitated by pulsing with probe ultrasonicator every 2-4 hours. Aliquots of samples $(2 \mathrm{~mL})$ were collected at definite time intervals $(0-168 \mathrm{~h})$ and the arsenic concentration in solution was monitored as a function of time.

Arsenic concentrations were measured using ICP-MS after the nanoparticles were removed from aqueous phase. Centrifugation and filtration were investigated for particle removal efficiency. The centrifugation speed and time as well as filter pore sizes and membrane materials were investigated. Nanoparticle suspension $(100 \mathrm{mg} / \mathrm{L})$ underwent centrifugation at 6,000 and $10,000 \mathrm{rpm}$ for 30 and 60 minutes. Filtration of the nanoparticle suspension was performed using as well filtration using $0.45 \mu \mathrm{m}$ PVDF filter, $0.22 \mu \mathrm{m}$ PVDF filter and $0.2 \mu \mathrm{m}$ nylon filter. The supernatant or filtrate was tested for any residual nanoparticles by measuring for total Fe using GFAAS.

The aliquots collected at the different time intervals $(0-168 \mathrm{~h})$ during the adsorption experiments were centrifuged for 60 minutes at 10,000 rpm. The supernatant was analyzed for arsenic concentrations using ICP-MS. The amount of arsenic adsorbed was calculated from the difference between the initial and supernatant arsenic concentrations. The IONPs particle size was monitored during the adsorption experiment. IONPs concentration was also determined by measuring the total iron concentration in the suspensions using GFAAS in order to monitor the sedimentation of IONPs over time. Before iron analysis, IONPs aliquots underwent acid digestion in accordance to EPA 
method 3050B. The IONPs were digested in nitric acid in the ratio of $1: 1$ at $95 \pm 5^{\circ} \mathrm{C}$ for 20 minutes using a heating block (Environmental Express). The digestate was made up to $50 \mathrm{~mL}$ with distilled deionized water and undergo further dilutions that are appropriate for GFAAS analysis.

Adsorption experiments on hematite aggregates were performed with similar procedures as described above except the hematite aggregates were dispersed using vortex for 20 minutes at level 10 which is the highest power setting of the vortex. In this study, iron oxides dispersed by vortex are referred to as hematite aggregates. This dispersion method generates particles in the micrometer size range (see chapter 2). The As-hematite aggregates samples were agitated by shaking on an orbital shaker at $250 \mathrm{rpm}$ for the entire experimental time.

\subsubsection{Adsorption Isotherms}

Hematite nanoparticles were dispersed similarly to procedures in the adsorption kinetics experiment. The hematite nanoparticles concentration was fixed at $10 \mathrm{mg} / \mathrm{L}$ and it was mixed with the appropriate amount of $10 \mathrm{mg} / \mathrm{L} \mathrm{As} \mathrm{(V)} \mathrm{or} \mathrm{As} \mathrm{(III)} \mathrm{stock} \mathrm{solutions}$ to vary the arsenic concentrations from 10-2000 $\mu \mathrm{g} / \mathrm{L}$. The As-IONPs dispersions were reacted to equilibrium (time $24 \mathrm{~h}$ determined from kinetic experiment). The dispersions were agitated by pulsing with probe sonicator every 2-4 hours at room temperature (20 $\left.{ }^{\circ} \mathrm{C}\right)$. At equilibrium, $2 \mathrm{~mL}$ aliquots of samples were collected and the IONPs were separated from aqueous phase by centrifuging at 10,000 rpm for 60 minutes. Total arsenic concentrations in the supernatant were measured using ICP-MS. Additional $2 \mathrm{~mL}$ aliquots of samples were collected at equilibrium and the iron concentration was measured. Isotherm experiments on hematite aggregates were performed with similar 
procedures as described above except the IONPs were dispersed using vortex. Samples were agitated by shaking on an orbital shaker at $250 \mathrm{rpm}$ for the entire experimental time.

\subsection{Results and Discussion}

\subsubsection{Characterization of hematite nanoparticles and hematite aggregates}

Figure 3.1 shows the SEM micrographs of hematite nanoparticles and hematite aggregates. Individual hematite nanoparticles, spherical in shape, can be seen with size ranges of 50-130 $\mathrm{nm}$ (Figure 3.1a).
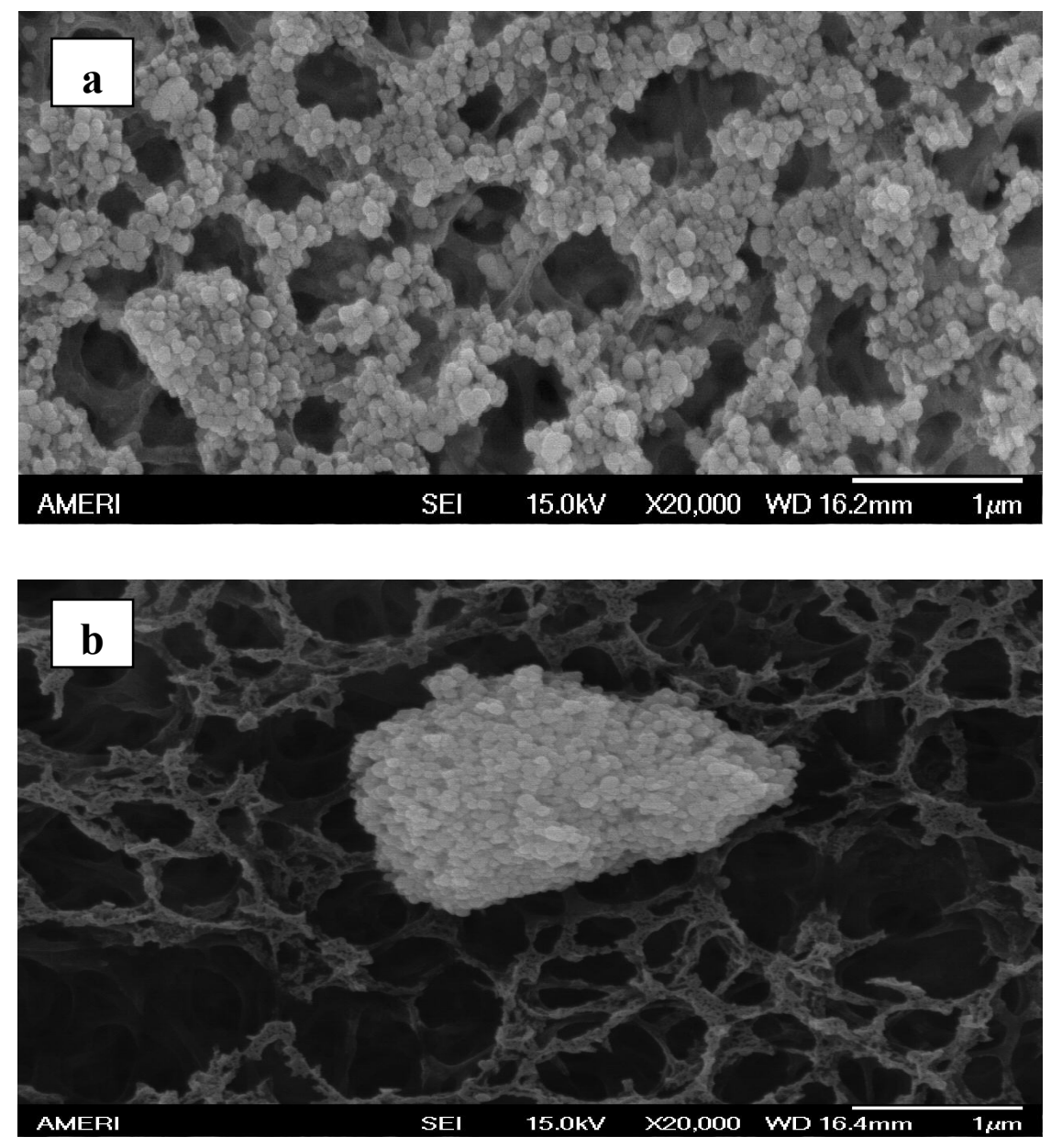

Figure 3. 1 SEM micrographs of (a) hematite nanoparticles $(10 \mathrm{mg} / \mathrm{L})$ in water dispersed using probe ultrasonication (b) hematite aggregates $(10 \mathrm{mg} / \mathrm{L})$ dispersed using vortex. 
This indicates that probe ultrasonication was effective in preparing nanoparticulate hematite dispersions. In the nanoparticle dispersion, there was a high concentration of individual particles in the nanosized range that are available for absorption. On the other hand, Figure $3.1 \mathrm{~b}$ shows a large hematite aggregate after dispersion by vortex. The aggregate is composed of numerous nanoparticles clumped together into a large mass with diameter averaging approximately $2.5 \mu \mathrm{m}$. The number of particles available for adsorption are much fewer compared to dispersion by probe ultrasonication as most of the individual particles are part of the large aggregate

\subsubsection{Adsorption Experiments}

\subsubsection{Removal of nanoparticles from aqueous phase}

In order to complete adsorption experiments, the nanoparticles have to be removed from the aqueous phase prior to the quantitative analysis of arsenic. Two techniques (centrifugation and filtration) were investigated for efficiency of hematite nanoparticles removal. Figure 3.2 shows the concentration of hematite nanoparticles that remain in the aqueous phase after centrifugation. Centrifugation at $6,000 \mathrm{rpm}$ for 30 minutes resulted in $1036 \mu \mathrm{g} / \mathrm{L}$ of IONPs remaining in the supernatant suggesting that $89 \%$ of IONPs was removed from the aqueous phase. An increase in the centrifugation time to 60 minutes while keeping the speed constant at 6,000 rpm resulted in lower IONPs concentration $(192.1 \mu \mathrm{g} / \mathrm{L})$ in the supernatant, a $98 \%$ removal of IONPs from aqueous phase. An increase in centrifugation speed to $10,000 \mathrm{rpm}$ for 30 minutes resulted in $1275 \mu \mathrm{g} / \mathrm{L}$ of IONPs remaining in the supernatant, $87 \%$ removal from aqueous phase (Figure 3.2). However, an increase in the centrifugation time to 60 minutes while maintaining a constant speed $(10,000 \mathrm{rpm})$ resulted in a significant lower concentration 
of IONPs $(8.04 \mu \mathrm{g} / \mathrm{L})$, a $99.9 \%$ removal from aqueous phase. A comparison of the results from varying the centrifugation speed and time showed that high speed of centrifugation $(10,000 \mathrm{rpm})$, whether for 30 minutes or 60 minutes of centrifugation, can remove $98 \%$ or more of IONPs from aqueous phase. However, centrifugation for 60 minutes yields the highest IONPs removal efficiency.

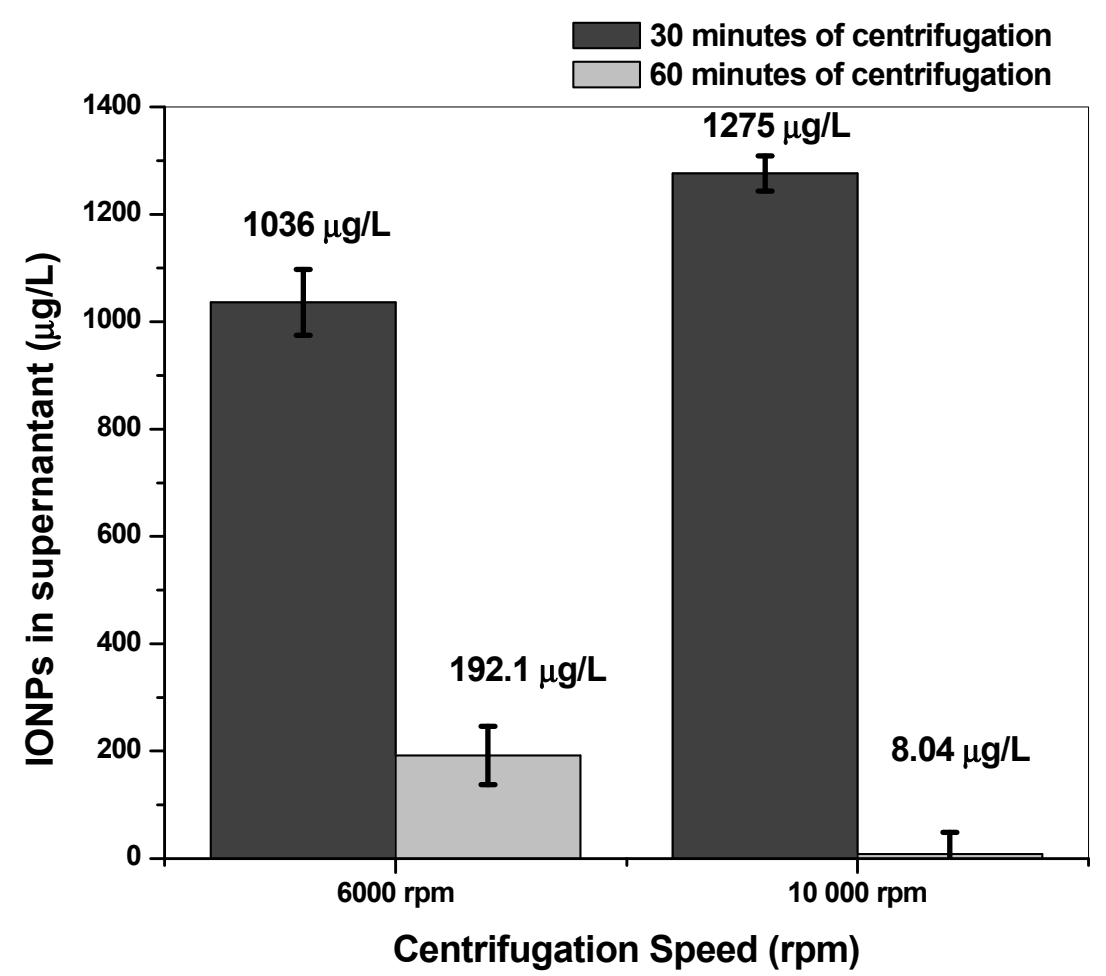

Figure 3. 2 The effect of centrifugation on the separation of IONPs from aqueous phase. A $10 \mathrm{mg} / \mathrm{L}$ IONPs dispersion was centrifuged at 10,000 and 6,000 rpm for 30 and 60 minutes.

Figure 3.3 shows the concentration of hematite nanoparticles remaining in the filtrate after filtration using filters of varying pore sizes and materials. Nanoparticles were able to penetrate through the filter as hematite nanoparticles were found in the filtrate. Filtration using $0.45 \mu \mathrm{m}$ PVDF filter, $0.22 \mu \mathrm{m}$ PVDF filter and $0.2 \mu \mathrm{m}$ nylon filter 
resulted in 2,430, 1,530, and $796 \mu \mathrm{g} / \mathrm{L}$ IONPs in the filtrate, respectively. These results suggest a $76 \%, 85 \%$ and $92 \%$ removal efficiency for the $0.45 \mu \mathrm{m}$ PVDF filter, $0.22 \mu \mathrm{m}$ PVDF filter and $0.2 \mu \mathrm{m}$ nylon filter, respectively, IONPs in the filtrate have an average particle size of $124.7 \pm 0.5 \mathrm{~nm}$ (typical size of bare IONPs dispersed using the probe ultrasonication method, see Chapter 2).

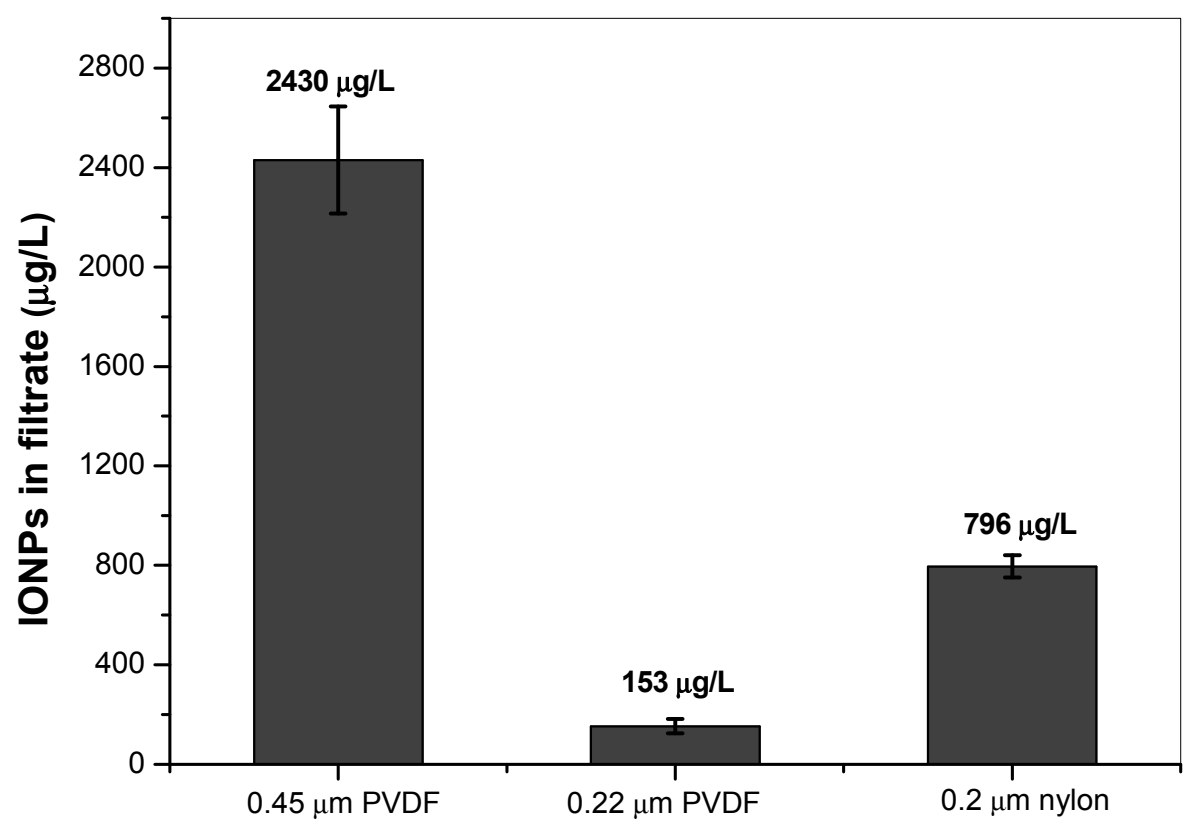

Filter pore size and membrane

Figure 3. 3 The effect of filtration on the separation of IONPs from aqueous phase. A 10 $\mathrm{mg} / \mathrm{L}$ IONPs dispersion was filtered using $0.45 \mu \mathrm{m}$ PVDF, $0.22 \mu \mathrm{m}$ PVDF and $0.22 \mu \mathrm{m}$ nylon filter.

These results indicated that a decrease in the pore sizes of the filter results in higher amounts of IONPs being retained on the filter and removed from the aqueous phase. However, considerable amounts of IONPs were able to penetrate through the filter 
as some nanoparticles were detected in the filtrate. From these results, centrifugation and filtration can remove most hematite nanoparticles from aqueous phase. A comparison of both techniques indicated that centrifugation at $10,000 \mathrm{rpm}$ for 60 minutes is more efficient at removing hematite nanoparticles from the aqueous phase than filtration. Therefore, centrifugation was used in this study to separate IONPs from the aqueous phase. These results are important as there is ambiguity in the literature in regards to the technique used for nanoparticle removal before instrumentation analysis. Numerous articles in the literature mentioned the use of centrifugation (Giasuddin et al., 2007; Auffan et al., 2008; Turk et al., 2010) and filtration (0.45 $\mu \mathrm{m}$ filter) (Kanel Sushil et al., 2005; Morgada et al., 2009; Shipley et al., 2009; Shipley et al., 2010a) to remove nanoparticles from aqueous phase but they failed to mention the efficiency of the removal. Any nanoparticles remaining in the aqueous phase can affect instrumentation analysis as well as interfering with the adsorption experiments.

\subsubsection{Adsorption of arsenite and arsenate to hematite nanoparticles}

The adsorption of $\mathrm{As}(\mathrm{III})$ and $\mathrm{As}(\mathrm{V})$ to hematite nanoparticles was investigated at $\mathrm{pH} 7-8.0$ which is the characteristic $\mathrm{pH}$ of many potable waters. Figures $3.4 \mathrm{a}$ and $3.4 \mathrm{~b}$ show the adsorption curve of $\mathrm{As}(\mathrm{III})$ and $\mathrm{As}(\mathrm{V})$ with hematite nanoparticles, respectively. Rapid adsorption was observed for both $\mathrm{As}(\mathrm{III})$ and $\mathrm{As}(\mathrm{V})$ to hematite nanoparticles within the first $8 \mathrm{~h}$ after which the adsorption rate slowed and equilibrium was attained at $\sim 24 \mathrm{~h}$. During the adsorption experiment, a slight drift in $\mathrm{pH}$ was observed (Table 3.1). There was no particular trend in $\mathrm{pH}$ variation as the $\mathrm{pH}$ increases and decreases randomly while remaining in the $\mathrm{pH} 7-8.0$ range throughout the experiment. These $\mathrm{pH}$ changes did not affect the adsorption studies as arsenic continued to adsorb to hematite nanoparticles 
in increasing amounts during the first $24 \mathrm{~h}$ although the $\mathrm{pH}$ increases and decreases randomly during that time. The slight change in $\mathrm{pH}$ could be attributed to the samples being not buffered.
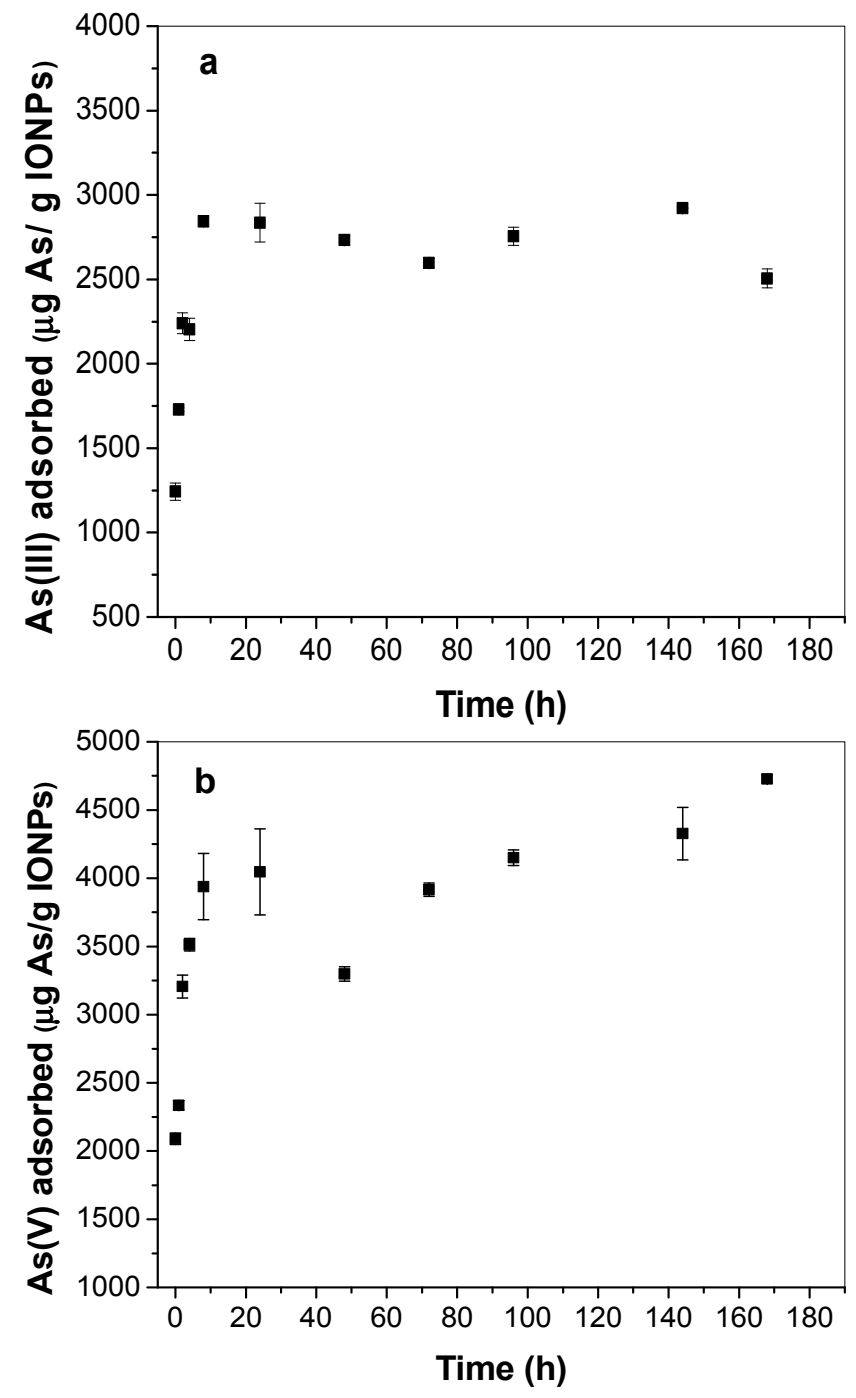

Figure 3. 4 Plots of arsenic adsorbed onto hematite nanoparticle. a) As(III) adsorbed onto hematite nanoparticles ( $\mu$ g As(III)/g IONPs) b) As(V) adsorbed onto hematite nanoparticles ( $\mu \mathrm{g}$ As(V)/g IONPs). Adsorption was performed using $200 \mu \mathrm{g} / \mathrm{L}$ As(III) or $\mathrm{As}(\mathrm{V})$ and $10 \mathrm{mg} / \mathrm{L}$ hematite nanoparticles over a contact time of $168 \mathrm{~h}$. 
Table 3. $1 \mathrm{pH}$ measurements during $\mathrm{As}(\mathrm{III})$ and $\mathrm{As}(\mathrm{V})$ adsorption to hematite nanoparticles over a contact time of $168 \mathrm{~h}$.

\begin{tabular}{ccc}
\hline Time (h) & As(III) & As(V) \\
\hline 0 & 7.96 & 7.52 \\
1 & 7.25 & 7.85 \\
2 & 7.66 & 7.74 \\
4 & 7.76 & 6.86 \\
8 & 7.61 & 7.20 \\
24 & 7.81 & 7.28 \\
48 & 7.68 & 7.72 \\
72 & 7.27 & 7.57 \\
96 & 7.62 & 7.89 \\
144 & 7.83 & 7.31 \\
168 & 7.22 & 7.57 \\
\hline
\end{tabular}

A kinetic fit of the data for the first $24 \mathrm{~h}$ was performed using a pseudo-second order kinetic model to determine initial rate constants and the amount of arsenic adsorbed at equilibrium. The pseudo second order kinetic model (equation 1a) is used to describe chemical adsorption, in particular, metal sorption onto different sorbents (Ho and McKay, 1999). In order to compare the adsorption of $A s(I I I)$ and $A s(V)$ to hematite nanoparticles, only the first $24 \mathrm{~h}$ data was used during the kinetic analysis. As(III) and $\mathrm{As}(\mathrm{V})$ both undergo rapid adsorption during the first $24 \mathrm{~h}$ after which the adsorption slowed for As(III) 
but desorption was observed for $\mathrm{As}(\mathrm{V})$. The factors causing $\mathrm{As}(\mathrm{V})$ desorption is uncertain at this time but it might be related to $\mathrm{As}(\mathrm{V})$ redox transformation (see Chapter 4). Never the less, the desorption of $A s(V)$ can affect the rate parameters, therefore, only the initial rates of adsorption are compared in this study. The pseudo second-order kinetic model for adsorption is expressed as (Ho et al., 1999; Azizian, 2004):

$\frac{d q}{d t}=k_{2}\left(q_{e}-q_{t}\right)^{2}$

Equation 1a can be integrated for the boundary conditions $t=0$ to $t=t$ and $q=0$ to $q=t$ to give equation $1 \mathrm{~b}$ (Lin et al., 2009). Equation $1 \mathrm{~b}$ can be rearranged to the linear form (equation 1c) where a plot of $\mathrm{t} / q_{t}$ versus $t$ can provide the pseudo second order rate constants by using the linear least-squares regression to estimate the parameters and their errors.

$$
\begin{aligned}
& \frac{1}{\left(q_{e}-q_{t}\right)}=\frac{1}{q_{e}}+k_{2} t . \\
& \frac{t}{q_{t}}=\frac{1}{k_{2} q_{e}^{2}}+\frac{t}{q_{e}}
\end{aligned}
$$

where $\mathrm{k}_{2}$ is the rate constant of sorption, $\mathrm{q}_{\mathrm{e}}$ is the amount of adsorbate at equilibrium $(\mu \mathrm{g} / \mathrm{g})$ and $\mathrm{q}_{\mathrm{t}}$ is the amount of adsorbate at a certain time $(\mu \mathrm{g} / \mathrm{g})$. A plot of $t / q_{t}$ versus $t$ provides a straight line with slope of $1 / k_{2} q_{e}^{2}$ and intercept of $1 / q_{e}$. The values of $\mathrm{k}_{2}$ and $\mathrm{q}_{\mathrm{e}}$ can be determined from the slope and intercept respectively. All data analysis and graphing were performed using the software OriginPro8. Figure 3.5 shows the pseudo 
second order kinetic for the adsorption of As(III) to hematite nanoparticles (Figure 3.5a) and $\mathrm{As}(\mathrm{V})$ adsorption to hematite nanoparticles (Figure 3.5b).
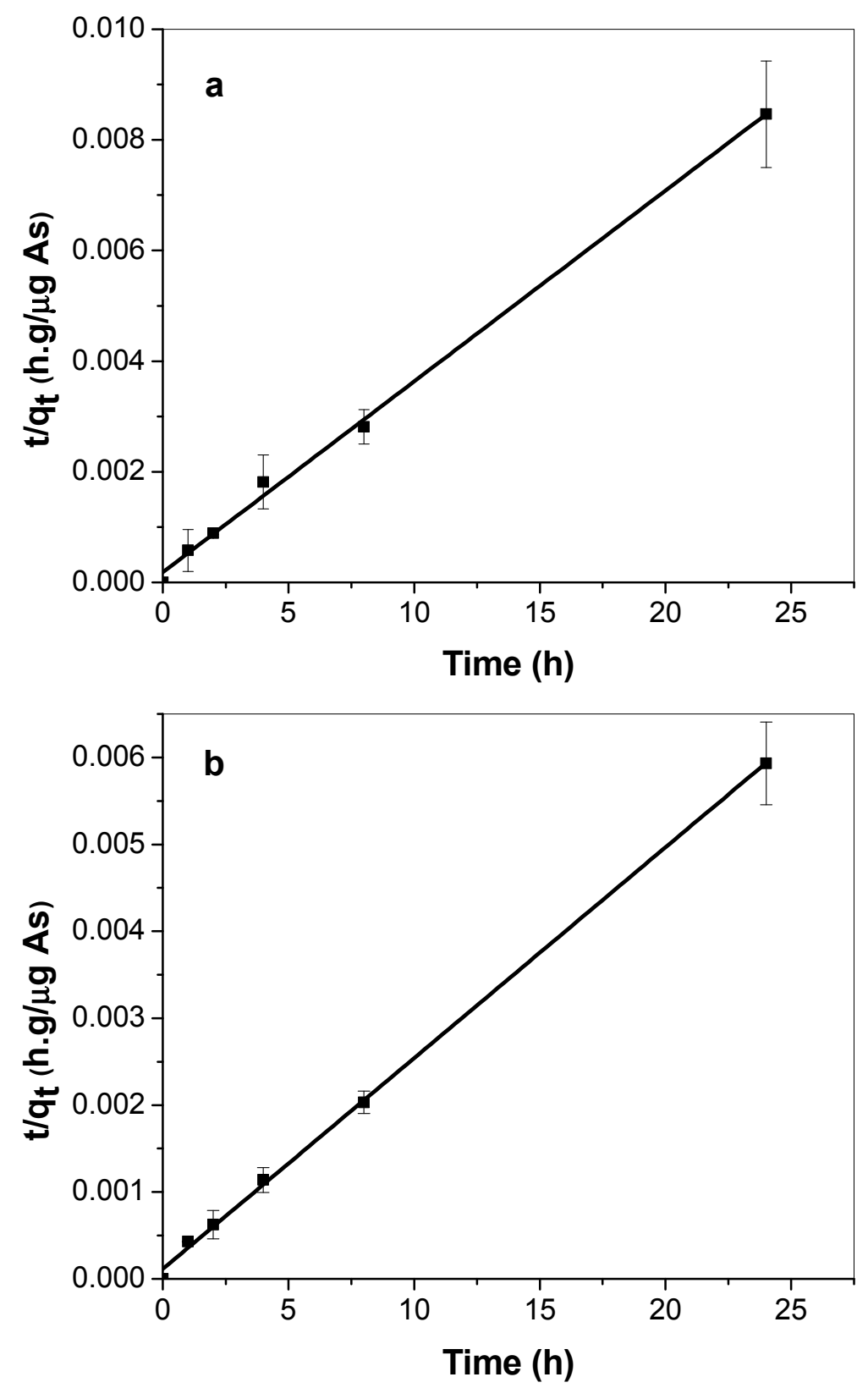

Figure 3. 5 Linear Pseudo-Second Order Kinetic Fit for the adsorption of (a) As(III) to hematite nanoparticles and (b) $\mathrm{As}(\mathrm{V})$ to hematite nanoparticles. 
From figure 3.5, there is a good linear fit for the second-order kinetic model for adsorption as $\mathrm{R}^{2}>0.99$. A pseudo first order kinetic fit was also performed on the data but the data did not fit the model appropriately. Using the pseudo second-order kinetic, the initial rate constant for $\mathrm{As}(\mathrm{V})$ adsorption is $5.10 \times 10^{-4} \pm 1.4 \times 10^{-4} \mathrm{~g} / \mu \mathrm{g} . \mathrm{h}$ and 6.45 $\mathrm{X} 10^{-4} \pm 3.1 \times 10^{-4} \mathrm{~g} / \mu \mathrm{g} . \mathrm{h}$ for As(III) adsorption (Table 3.2). There was no significant difference between the adsorption rate for As(III) and As(V) onto hematite nanoparticles ( $\mathrm{z}$-test, $\mathrm{p}>0.05)$. However, a larger amount of $\mathrm{As}(\mathrm{V})$ can be adsorbed at equilibrium $\left(\mathrm{q}_{\mathrm{e}}\right.$ $=4,122 \pm 63 \mu \mathrm{g} / \mathrm{g})$ compared to As(III) $\left(\mathrm{q}_{\mathrm{e}}=2,899 \pm 71 \mu \mathrm{g} / \mathrm{g}\right)($ Table 3.2).

Table 3. 2 Kinetic (pseudo second order) parameters for the adsorption of As(III) and As(V) with hematite nanoparticles and hematite aggregates.

\begin{tabular}{llccc}
\hline Particle & Arsenic & $\mathbf{Q}_{\mathbf{e}}(\boldsymbol{\mu g} / \mathbf{g})$ & $\mathbf{k}_{\mathbf{2}}(\mathbf{g} / \boldsymbol{\mu g} . \mathbf{h})\left(\mathbf{1 0}^{\mathbf{- 4}}\right)$ & $\mathbf{R}^{\mathbf{2}}$ \\
\hline Hematite & As (III) & $2899 \pm 71$ & $6.45 \pm 3.1$ & 0.9970 \\
nanoparticles & As (V) & $4122 \pm 63$ & $5.10 \pm 1.2$ & 0.9988 \\
& & & & \\
\hline Hematite & As (III) & $1689 \pm 90$ & $3.82 \pm 1.4$ & 0.9961 \\
aggregates & As (V) & $2614 \pm 73$ & & 0.9859 \\
& & & & \\
\hline
\end{tabular}

The reactivity and the adsorption capabilities of hematite nanoparticles can be affected by the aggregation/growth of the particle from nanoparticulate sizes to micrometer size particles. Therefore, to determine if aggregation of the nanoparticles during the adsorption experiments affects the adsorption process, the hydrodynamic 
diameter of the particles was monitored during the adsorption experiments. The $\mathrm{t}_{0 \mathrm{~h}}$ hydrodynamic sizes, although slightly outside the range of what is considered nanoparticles $(<100 \mathrm{~nm})$, are the minimum sizes that can be achieved using the probe ultrasonication dispersion method. From our previous work (Dickson et al., 2012) and other's (Zhang et al., 2008), the dispersion of commercial nanoparticles to the originally acclaimed size by the manufacturer $(20-50 \mathrm{~nm})$ is difficult. The probe ultrasonicator cannot break down IONPs to its primary particle diameter due to possible aggregation of the particles during synthesis or long periods of storage.

Figures 3.6a and 3.6b (triangles) show the particle size distribution during the adsorption of $\mathrm{As}(\mathrm{III})$ and $\mathrm{As}(\mathrm{V})$ respectively to hematite nanoparticles. During the period of rapid adsorption ( $0-8 \mathrm{~h})$, the hydrodynamic diameter of hematite nanoparticles increased as $\mathrm{As}(\mathrm{III})$ and $\mathrm{As}(\mathrm{V})$ adsorb to the hematite nanoparticle surface (Figs 3.6a and 3.6b triangles). In the presence of As(III), the hematite nanoparticle size increased from $122 \mathrm{~nm}$ at $\mathrm{t}_{0}$ to $213 \mathrm{~nm}$ at $\mathrm{t}_{8 \mathrm{~h}}$ but reduced to sizes similar to the control (120-160 nm) from $\mathrm{t}_{24-168 \mathrm{~h}}$. In the presence of $\mathrm{As}(\mathrm{V})$, the particle size drastically increased from $137 \mathrm{~nm}$ at $\mathrm{t}_{0}$ to $345 \mathrm{~nm}$ at $\mathrm{t}_{2 \mathrm{~h}}$ but reduced to sizes similar to the control $(120-160 \mathrm{~nm})$ from $\mathrm{t}_{24-168 \mathrm{~h}}$. These changes in size are possibly due to the adsorption of arsenic to the hematite nanoparticle surface causing a change in the surface charge and increasing particle growth. Researchers studying the adsorption of arsenic to bulk ferrihydrite (FeOOH) had found that there is a reduction in the surface charge of ferrihydrite when both arsenite and arsenate adsorb (Jain et al., 1999). Therefore, the surface charge properties of hematite nanoparticles could explain, at least partially, the aggregation of hematite nanoparticles when arsenic is adsorbed. At this $\mathrm{pH}\left(\mathrm{pH}\right.$ 6-8), $\mathrm{As}(\mathrm{III})$ is neutral $\left(\mathrm{H}_{3} \mathrm{AsO}_{2}{ }^{0}\right)$ and hematite 
nanoparticle is slightly positively charged with a zeta potential $+29.8 \pm 0.917 \mathrm{mV}$ (see Chapter 2).
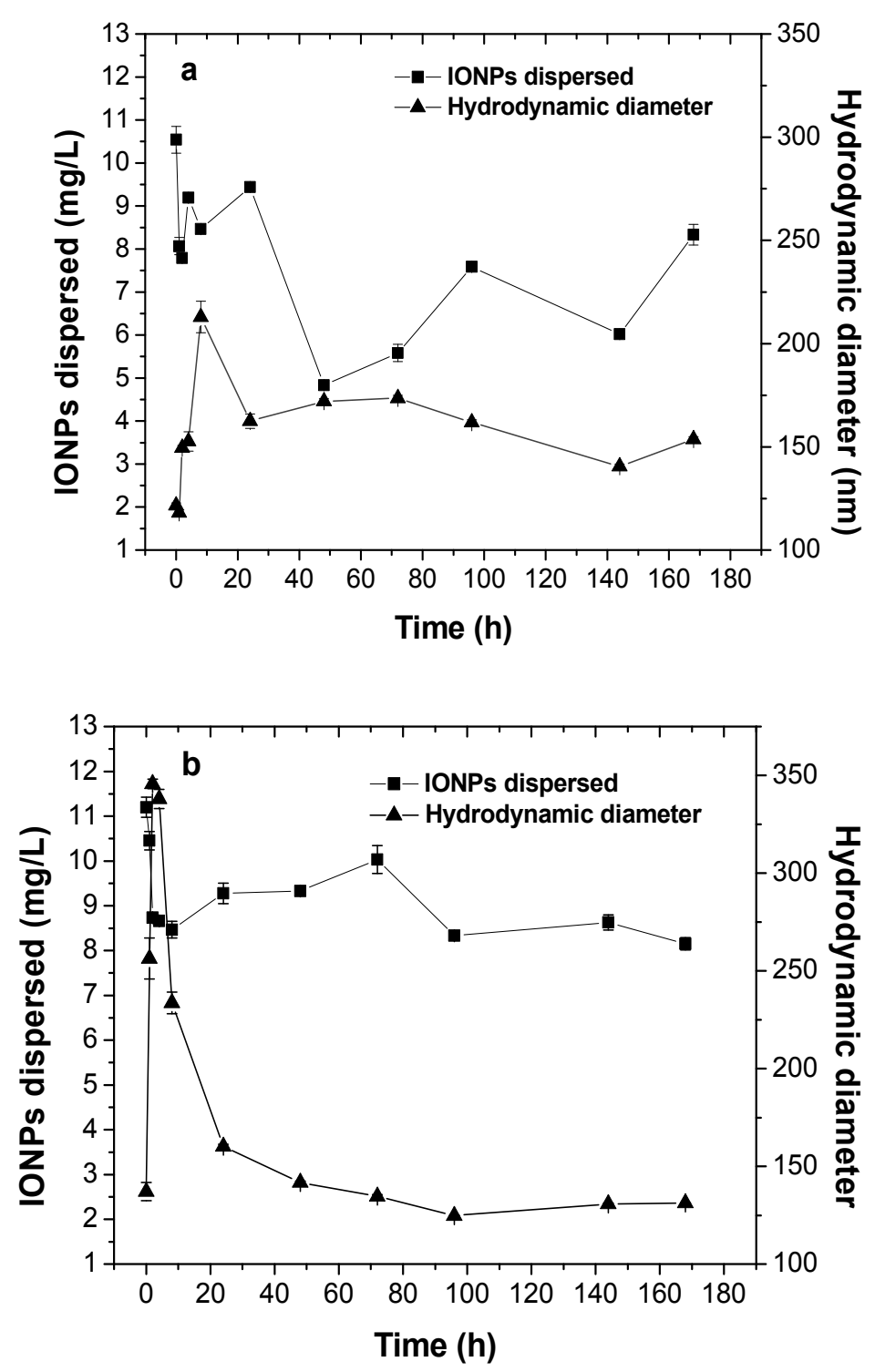

Figure 3. 6 Hematite nanoparticle concentration (squares) and particle size distribution (triangles) monitored during adsorption (contact time of 168h) (a) As(III) and (b) As(V). 
There is a possibility that the surface charge of hematite nanoparticle becomes suppressed when arsenite adsorbs causing the aggregation and sedimentation of the particles as evidenced by a reduction in the amount of IONPs in the dispersion from $t_{0}$ to $t_{8}$ (Figure 3.6a squares). On the other hand, $\mathrm{As}(\mathrm{V})$ is negatively charged $\left(\mathrm{H}_{2} \mathrm{AsO}_{4}{ }^{-}\right.$and $\left.\mathrm{HAsO}_{4}{ }^{2-}\right)$ at pH 6-8 and there is a strong electrostatic attraction between $\mathrm{As}(\mathrm{V})$ and the slightly positively charged nanoparticle. Therefore, the charge becomes neutralized during adsorption and this caused the particles to aggregate and sediment as evidenced by a reduction in the amount of hematite nanoparticles in the dispersion from $\mathrm{t}_{0}$ to $\mathrm{t}_{8 \mathrm{~h}}$ (Figure 3.6b squares). After $8 \mathrm{~h}$, adsorption of arsenate and arsenite is minimal and the IONPs particle sizes are similar to those of the control $(120-160 \mathrm{~nm})$ with minimal sedimentation suggesting that the IONPs that remained in suspension is not bound/affected by arsenic. The suppression or neutralization of the charge once arsenic adsorbs to hematite nanoparticles reduces any electrostatic repulsion between the nanoparticles causing them to come together and aggregate. The results of these adsorption experiments suggest that $\mathrm{As}(\mathrm{V})$ is more readily adsorbed to hematite nanoparticles than As(III). The negatively charged $\mathrm{As}(\mathrm{V})$ has a stronger electrostatic attraction to the positively charged hematite particles than the electrically neutral As(III). Therefore, the surface charge properties of both the hematite nanoparticle and arsenic is a good explanation as to why higher amounts of $\mathrm{As}(\mathrm{V})$ than $\mathrm{As}(\mathrm{III})$ is adsorbed at equilibrium.

\subsubsection{Adsorption of arsenite and arsenate to hematite aggregates}

The adsorption of arsenic to hematite aggregates was investigated to determine the extent at which the reduction in surface area affects hematite reactivity and the efficiency of arsenic adsorption. Figures $3.7 \mathrm{a}$ and $3.7 \mathrm{~b}$ show the adsorption curve of 
$\mathrm{As}(\mathrm{III})$ and $\mathrm{As}(\mathrm{V})$ with hematite aggregates. There was rapid adsorption of $\mathrm{As}(\mathrm{III})$ and $\operatorname{As}(V)$ to hematite aggregates within the first $24 \mathrm{~h}$ after which arsenic desorption was

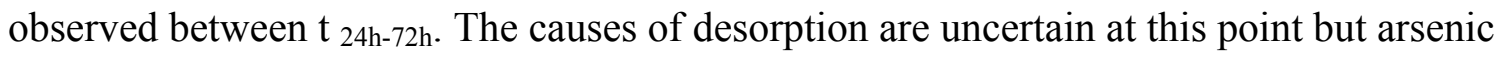
species transformation is one probable cause of arsenic release from the particle surface (Islam et al., 2004; Pedersen et al., 2006; Tufano et al., 2008).

A pseudo second order kinetic fit was carried out for the first $24 \mathrm{~h}$ for arsenic adsorption to hematite aggregates (Figures 3.8a and 3.8b). In order to compare arsenic adsorption on hematite aggregates to hematite nanoparticles, the kinetic fit was carried out for the first $24 \mathrm{~h}$. Similarly to $\mathrm{As}(\mathrm{V})$ adsorption to hematite nanoparticles, arsenic adsorption to hematite aggregates was observed during the first 24h after which desorption was observed. As mentioned before, the desorption of arsenic can possibly affect the rate parameters, therefore, only the initial rates of adsorption are compared in this study. The initial rate constants for adsorption are $4.60 \times 10^{-4} \pm 2.7 \times 10^{-4} \mathrm{~g} / \mu \mathrm{g} . \mathrm{h}$ for $\operatorname{As}(\mathrm{V})$ and $3.82 \times 10^{-4} \pm 1.4 \times 10^{-4} \mathrm{~g} / \mu \mathrm{g} . \mathrm{h}$ for As(III). These $\mathrm{k}_{2}$ values are similar as there was no significant difference between the adsorption rate for $\mathrm{As}(\mathrm{III})$ and $\mathrm{As}(\mathrm{V})$ to hematite aggregates (z-test, $\mathrm{p}>0.05$ ) suggesting that hematite aggregates were able to adsorb both $\mathrm{As}(\mathrm{III})$ and $\mathrm{As}(\mathrm{V})$ at similar rates. However, $\mathrm{As}(\mathrm{V})$ was adsorbed in larger amounts at equilibrium $\left(\mathrm{q}_{\mathrm{e}}=2,614 \pm 73 \mu \mathrm{g} / \mathrm{g}\right)$ compared to $\operatorname{As}(\mathrm{III})\left(\mathrm{q}_{\mathrm{e}}=1,689 \pm 90 \mu \mathrm{g} / \mathrm{g}\right)$ (Table 3.2). As mentioned in section 3.3.2.1, the surface charge properties is a good explanation as to why the negatively charged $\mathrm{As}(\mathrm{V})$ has a stronger electrostatic attraction to the positively charged hematite particles than the electrically neutral As(III). 

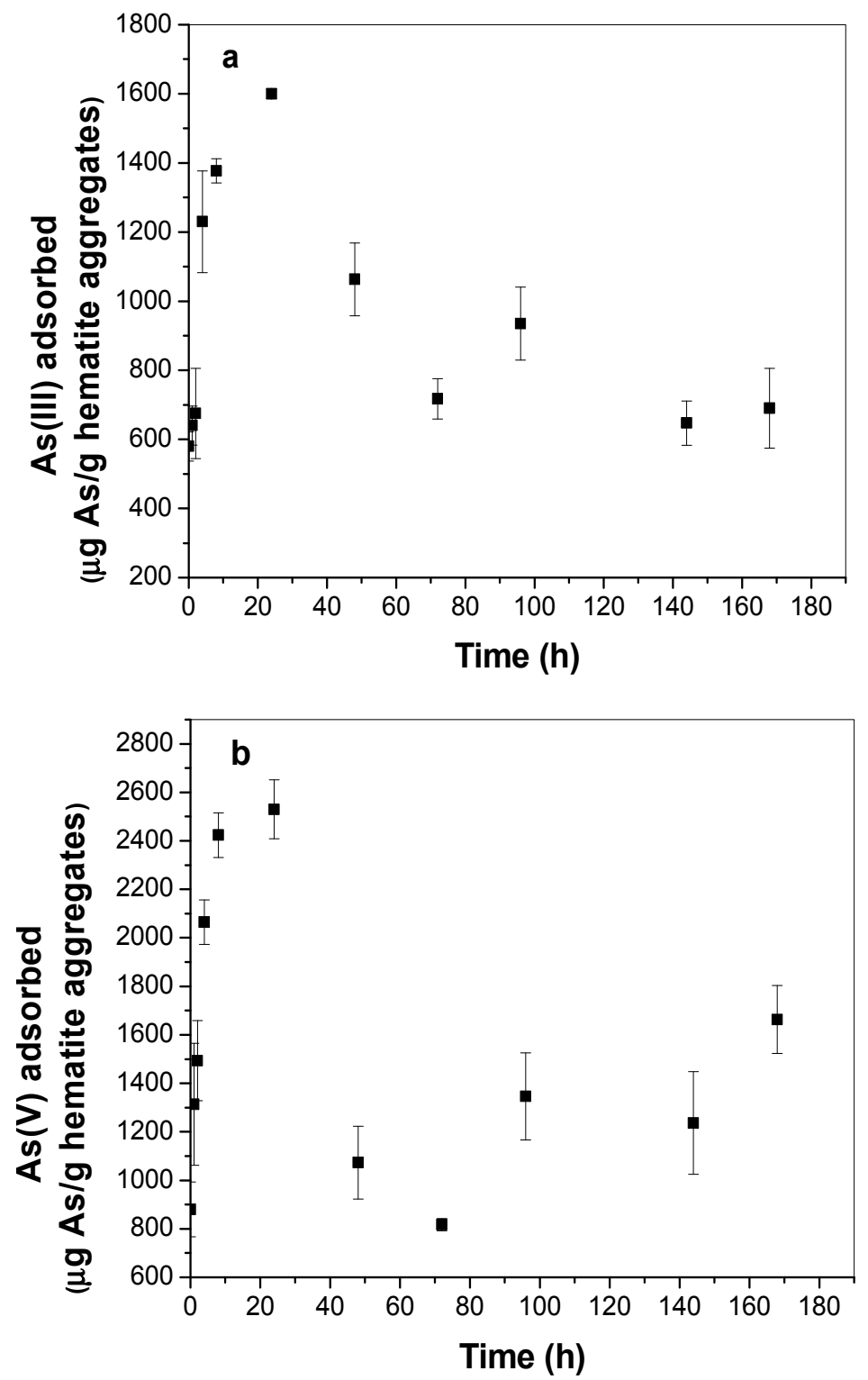

Figure 3. 7 Plots of arsenic adsorbed onto hematite aggregates. a) As(III) adsorbed onto hematite aggregates ( $\mu \mathrm{g} \mathrm{As}(\mathrm{III}) / \mathrm{g}$ IONPs) b) $\mathrm{As}(\mathrm{V})$ adsorbed onto hematite aggregates. Adsorption was performed using $200 \mu \mathrm{g} / \mathrm{L}$ As(III) and As(V) and $10 \mathrm{mg} / \mathrm{L}$ hematite aggregates over a contact time of $168 \mathrm{~h}$. 

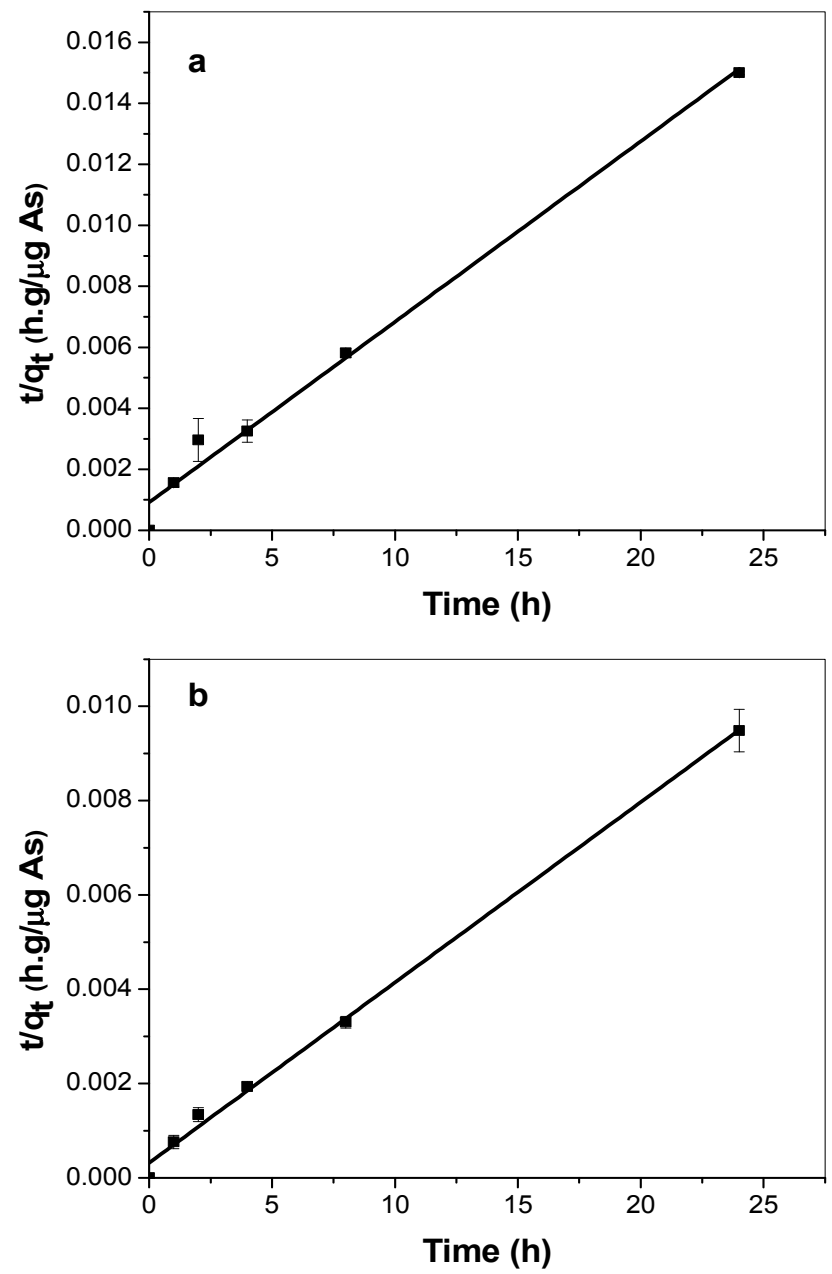

Figure 3. 8 Linear Pseudo-Second Order Kinetic Fit for the adsorption of (a) As(III) to hematite aggregates and (b) of $\mathrm{As}(\mathrm{V})$ to hematite aggregates.

\subsubsection{Particle size effect on the kinetics of adsorption}

A comparison of the results from the kinetic study showed that there was no significance difference between the initial rate of adsorption of $\mathrm{As}(\mathrm{III})$ and $\mathrm{As}(\mathrm{V})$ to hematite nanoparticles and hematite aggregates ( $\mathrm{z}$ test, $\mathrm{p}<0.05)$. This assumption was formed on the basis of the rate constants for the adsorption reactions. The fastest rate of arsenic (III, V) adsorption occurred in the first $24 \mathrm{~h}$ regardless of the particle size. 
However, the amount of arsenic adsorb at equilibrium was different depending on the size of the particle. The results indicated that hematite nanoparticles adsorbed significantly more $\mathrm{As}(\mathrm{III})$ and $\mathrm{As}(\mathrm{V})$ at equilibrium compared to hematite aggregates ( $\mathrm{z}$ test, $\mathrm{p}<0.05$ ). The FE-SEM imaging of the hematite nanoparticles (Figure 1a) showed large amounts of individual particles available for arsenic adsorption. The high concentration of individual nanoparticles suggests that there is more nanoparticle surface available to accommodate a high volume of arsenic adsorbing to the surface. On the other hand, the large aggregates are fewer in suspension and have smaller surface area resulting in a reduction in adsorption sites hence reducing its adsorption efficiency.

\subsubsection{Adsorption Isotherms}

\subsubsection{Arsenic adsorption isotherm to hematite nanoparticles}

From the kinetic studies, it is observed that hematite nanoparticles adsorb more arsenic at equilibrium compared to hematite aggregates. To understand the thermodynamics of adsorption, the Freundlich isotherm was used to describe the adsorption equilibrium data for the adsorption of $\mathrm{As}(\mathrm{V})$ and $\mathrm{As}(\mathrm{III})$ with hematite nanoparticles and aggregates. The purpose of the adsorption isotherm experiments is to understand the relationship between the adsorbate concentration and its degree of adsorption to the hematite surface (nanoparticles and aggregates) at equilibrium. The Freundlich model best describes adsorption equilibrium on heterogeneous materials and assumes that there is an infinite supply of adsorbent sites. The Freundlich isotherm is expressed as (Banerjee et al., 2008; Skopp, 2009; Duran et al., 2011):

$$
q_{e}=K_{f} C_{e}^{1 / n}
$$


where $\mathrm{q}_{\mathrm{e}}$ is the amount of As adsorbed at equilibrium ( $\mu \mathrm{g}$ As/g IONPs), $\mathrm{C}_{\mathrm{e}}$ is the equilibrium As concentration $(\mu \mathrm{g} / \mathrm{L}), \mathrm{K}_{\mathrm{f}}$ is the Freundlich constant for relative adsorption capacity ( $\mu \mathrm{g}$ As /g IONPs) and $\mathrm{n}$ is the Freundlich constants for adsorption intensity $(\mu \mathrm{g} / \mathrm{L})$.Figure 3.9 describes the Freundlich isotherm for the adsorption of As(III) and $\operatorname{As}(\mathrm{V})$ to hematite nanoparticles.
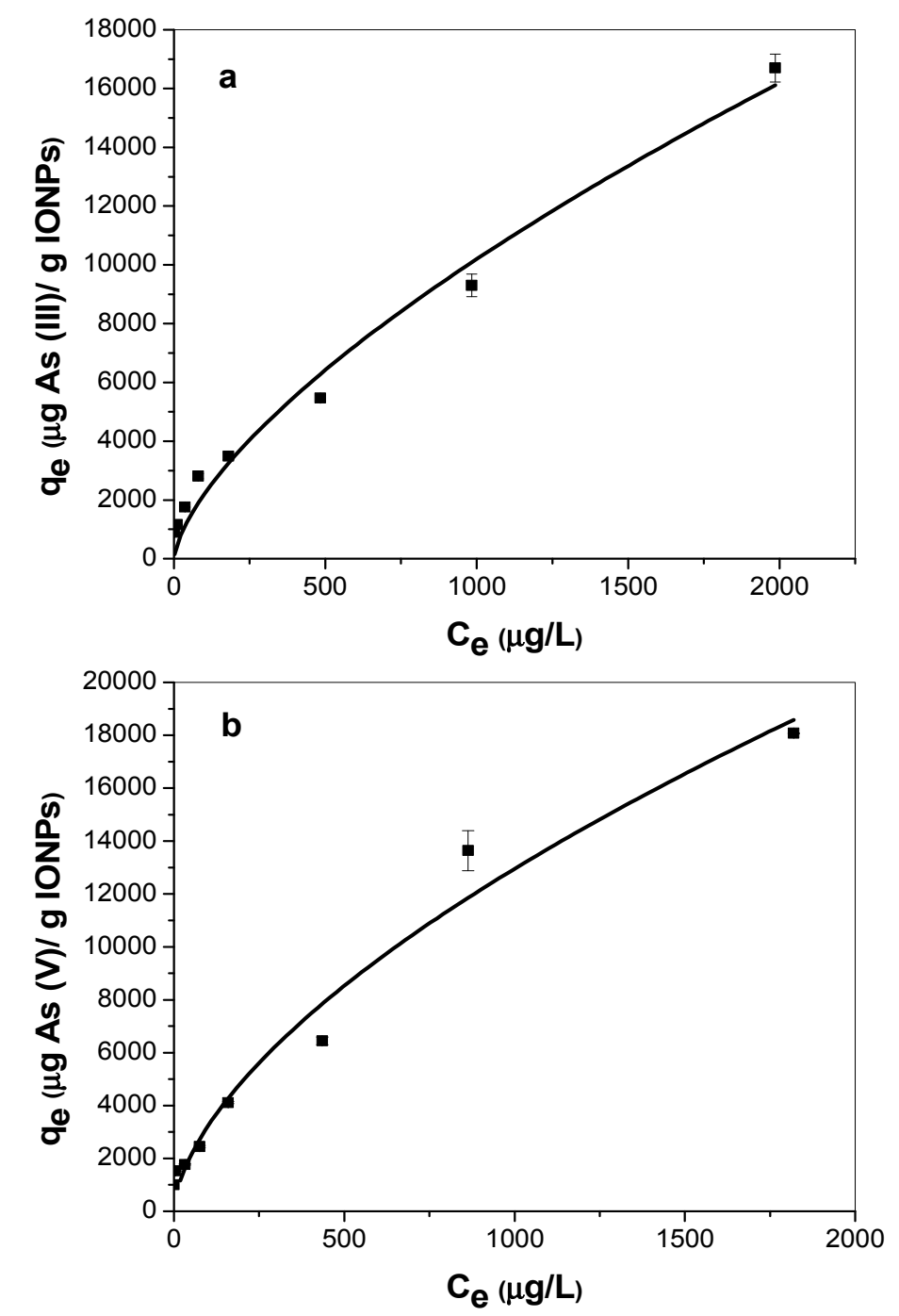

Figure 3. 9 Freundlich Isotherm of (a) As(III) and (b) As(V) with hematite nanoparticles. Arsenic concentrations vary from 10-2000 $\mu \mathrm{g} / \mathrm{L}$ and reacted with $10 \mathrm{mg} / \mathrm{L}$ of hematite nanoparticles to equilibrium (24h). 
The amount of arsenic adsorbing to the nanoparticle surface increased as the concentration of $\mathrm{As}(\mathrm{III})$ and $\mathrm{As}(\mathrm{V})$ in solution increased (Figure 3.9), indicating that there was no upper limit on adsorption under the experimental conditions and the Freundlich model was used appropriately to describe the equilibrium data. The data had a good fit to the Freundlich model $\left(\mathrm{R}^{2}>0.96\right)$. The parameters from the Freundlich isotherms are summarized in Table 3.3. The $\mathrm{K}_{\mathrm{f}}$ values for $\mathrm{As}(\mathrm{III})$ and $\mathrm{As}(\mathrm{V})$ are $101 \pm$ $44.0 \mu \mathrm{g} \mathrm{As} / \mathrm{g}$ IONPs and $200 \pm 92.2 \mu \mathrm{g}$ As/g IONPs, respectively, suggesting that hematite nanoparticles has a significantly higher adsorption capacity for $\mathrm{As}(\mathrm{V})$ compared to $\mathrm{As}(\mathrm{III})(\mathrm{z}$-test, $\mathrm{p}<0.05)$. The surface charge properties might explain why As(V) had a higher adsorption capacity than As(III). The negatively charged As(V) will have a stronger attraction to the slightly positive hematite nanoparticles and adsorbed more to the hematite nanoparticle hence the higher adsorption capacity. The neutrally charged As(III) has less attraction to hematite nanoparticle surface hence the lower adsorption capacity.

The $\mathrm{n}$ value is the Freundlich constants for adsorption intensity. It describes the degree of favorability of adsorption of one adsorbate to another. The ideal value of $n$ should be between 1 and 10 for favorable adsorption. For hematite nanoparticles $\mathrm{n}$ is $1.66 \pm 0.18 \mu \mathrm{g} / \mathrm{L}$ for $\mathrm{As}(\mathrm{V})$ and $1.50 \pm 0.13 \mu \mathrm{g} / \mathrm{L}$ for As(III). These results show that arsenic, whether As(V) or As (III), will naturally adsorb to hematite nanoparticles since arsenic have a strong binding affinity towards iron oxides. 
Table 3. 3 Freundlich isotherm parameters for the adsorption of As(III) and As(V) with hematite nanoparticles and hematite aggregates.

\begin{tabular}{lcccc}
\hline Particle & Arsenic & $\mathbf{K}_{\mathbf{f}}(\boldsymbol{\mu g} / \mathbf{g})$ & $\mathbf{n}(\boldsymbol{\mu g} / \mathbf{L})$ & $\mathbf{R}^{2}$ \\
\hline $\begin{array}{l}\text { Hematite } \\
\text { nanoparticles }\end{array}$ & As (III) & $101 \pm 44$ & $1.50 \pm 0.13$ & 0.9773 \\
& As (V) & $200 \pm 92$ & $1.66 \pm 0.18$ & 0.9689 \\
& As (III) & $26.8 \pm 8.7$ & $1.40 \pm 0.086$ & 0.9899 \\
\hline $\begin{array}{l}\text { Hematite } \\
\text { aggregates }\end{array}$ & As (V) & $21.5 \pm 14$ & $1.25 \pm 014$ & 0.9682 \\
& & & & \\
\hline
\end{tabular}

\subsubsection{Arsenic adsorption isotherm to hematite aggregates}

Figure 3.10 shows the adsorption isotherm for hematite aggregates. The Freundlich isotherm was also used to model the data which had a good fit to this model $\left(\mathrm{R}^{2}>0.96\right)$. The $\mathrm{K}_{\mathrm{f}}$ values for $\mathrm{As}(\mathrm{V})$ and $\mathrm{As}(\mathrm{III})$ are $21.5 \pm 14.4 \mu \mathrm{g}$ As/g IONPs and $26.8 \pm 8.74 \mu \mathrm{g}$ As/g IONPs respectively (Table 3.3). These results showed that there was no significant difference between the adsorption capacity for $\mathrm{As}(\mathrm{III})$ and $\mathrm{As}(\mathrm{V})$ for hematite aggregates (z-test, $p>0.05$ ). For hematite aggregates, the adsorption intensity ( $n$ values) for $\mathrm{As}(\mathrm{V})$ and $\mathrm{As}(\mathrm{III})$ are $1.25 \pm 0.14$ and $1.40 \pm 0.086 \mu \mathrm{g} / \mathrm{L}$, respectively. From these results, there were no significant difference between the favorability of adsorption of $\mathrm{As}(\mathrm{III})$ and $\mathrm{As}(\mathrm{V})$ to hematite aggregates (z-test, $\mathrm{p}>0.05)$. These isotherm parameters suggest that hematite aggregates can adsorb similar concentrations of $\mathrm{As}(\mathrm{V})$ and $\mathrm{As}(\mathrm{III})$ at equilibrium and it does not favor to adsorb one arsenic species over the other. 

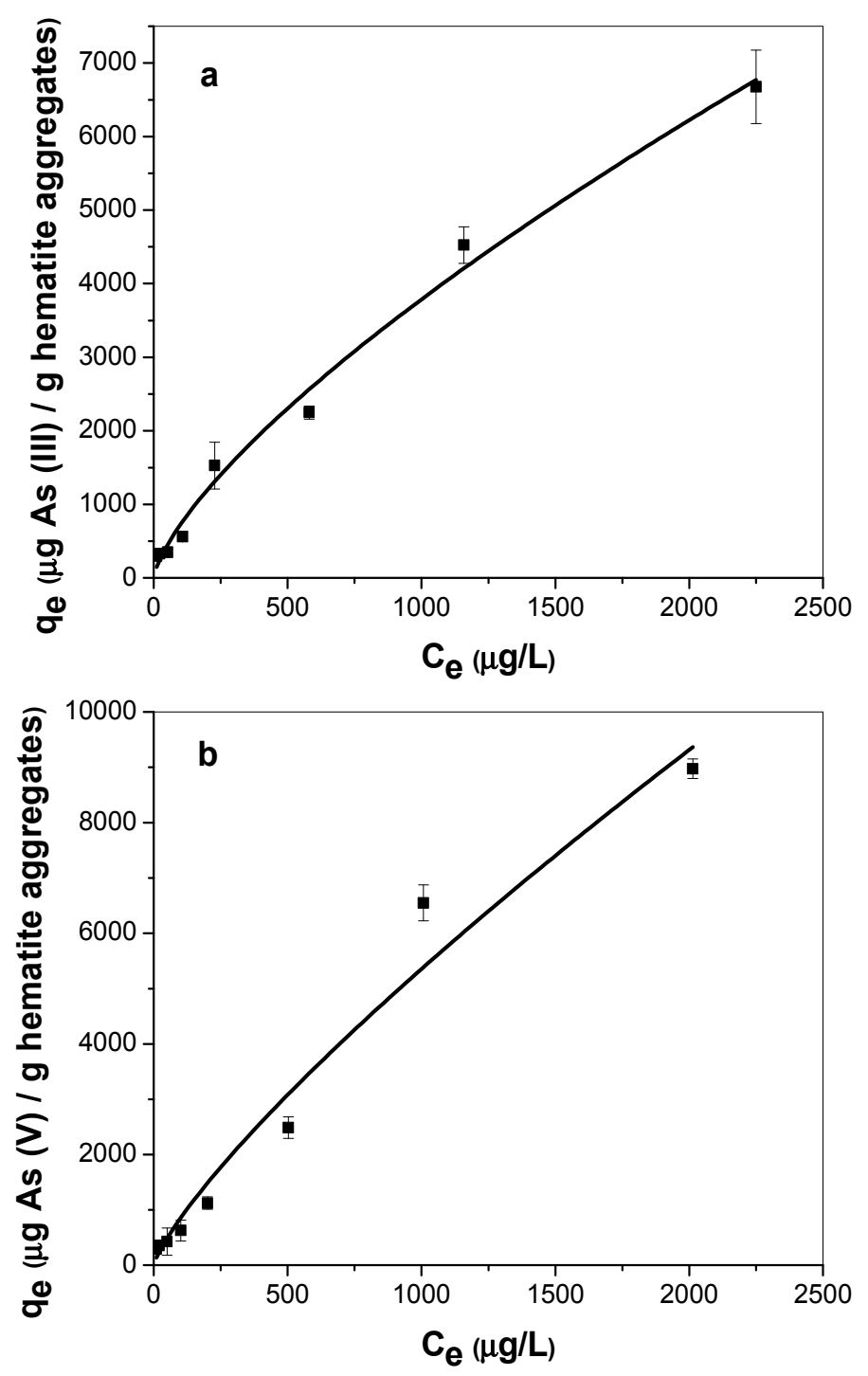

Figure 3. 10 Freundlich Isotherm of (a) As(III) and (b) As(V) with hematite aggregate. Arsenic concentrations vary from $10-2000 \mu \mathrm{g} / \mathrm{L}$ and reacted with $10 \mathrm{mg} / \mathrm{L}$ of hematite aggregates to equilibrium (24h). 


\subsubsection{Particle size effect on the thermodynamics of adsorption}

Isotherm studies showed that hematite has a favorable adsorption for both As(V) and As(III) regardless of the particle size. Therefore, arsenic will readily adsorb to hematite nanoparticles and hematite aggregates and the amount of arsenic adsorbing onto the hematite particle will increase as the concentration of arsenic increases in the solution. However, hematite nanoparticles have significantly higher adsorption capacity for both As(III) and As(V) compared to hematite aggregates (Table 3.3) (z-test, p < 0.05). The adsorption capacity for As(V) to hematite nanoparticle (200 $\pm 92 \mu \mathrm{g}$ As/g IONPs) is nine times higher than As(V) adsorption capacity for hematite aggregates $(21.5 \pm 14 \mu \mathrm{g} \mathrm{As} / \mathrm{g}$ IONPs ). A similar trend was observed for As(III) in which the adsorption capacity for As(III) to hematite nanoparticle (101 $\pm 44 \mu \mathrm{g}$ As/g IONPs) is approximately four times higher than As(III) capacity for hematite aggregates $(26.8 \pm 8.7 \mu \mathrm{g}$ As/g IONPs $)$. The higher adsorption capacity for the nanoparticles are a result of the nanoparticles being more reactive due to the higher amounts of adsorption sites compared to those of hematite aggregates. Therefore, the nanoparticles can accommodate larger amounts of arsenic adsorbing to their surface.

\subsubsection{Comparison to other's work}

Information on the kinetic and thermodynamic parameters of arsenic adsorption to hematite nanoparticles is lacking in the literature making it challenging to do a direct comparison. However, the results of this study are comparable to others' work where iron oxides nanoparticles (regardless of the mineral phase) can adsorb higher concentrations of arsenic compared to bulk particles (Table 3.4). From Table 3.4, the particles in the 
Table 3. 4 Arsenic adsorption capacity to iron oxide minerals

\begin{tabular}{|c|c|c|c|c|}
\hline \multirow[t]{2}{*}{$\begin{array}{l}\text { Forms of } \\
\text { Iron Oxide }\end{array}$} & \multirow[t]{2}{*}{ Particle Size } & \multicolumn{2}{|c|}{$\begin{array}{c}\text { Adsorption } \\
\text { Capacity (ug/g) }\end{array}$} & \multirow[t]{2}{*}{ References } \\
\hline & & As(III) & $\operatorname{As}(\mathbf{V})$ & \\
\hline Magnetite & $20 \mathrm{~nm}$ & --- & 1186 & (Shipley et al., 2009) \\
\hline NZVI & $10-100 \mathrm{~nm}$ & 3500 & --- & (Kanel et al., 2005) \\
\hline Maghemite & $18.4 \mathrm{~nm}$ & --- & 5000 & (Tuutijarvi et al., 2009) \\
\hline Granular ferric hydroxide & $0.32-2 \mathrm{~mm}$ & --- & 3130 & (Banerjee et al., 2008) \\
\hline iron oxide coated sand & --- & 690 & --- & (Kundu et al., 2007) \\
\hline $\mathrm{Fe}_{2} \mathrm{O}_{3}$ & $0.7 \mu \mathrm{m}$ & --- & 560 & (Jeong et al., 2007) \\
\hline Hematite nanoparticles & $120 \mathrm{~nm}$ & 2899 & 4122 & This study \\
\hline Hematite aggregates & $2.5 \mu \mathrm{m}$ & 1689 & 2614 & This study \\
\hline
\end{tabular}


nanometer range tend to adsorb higher amounts of arsenic regardless of the iron oxide mineral phase. In addition, this study is the first to report the aggregation of the nanoparticles during the adsorption experiment. No previous studies have investigated the change in size of the iron oxide particles during the adsorption process and how the adsorption of arsenic to the nanoparticles' surface can influence aggregation. This information is crucial as it suggests that the interaction of arsenic with the nanoparticle surface can change the IONPs surface charge and possibly increases aggregation of the nanoparticles.

\subsection{Conclusions}

The study was conducted to evaluate the adsorption of As(III) and As(V) to hematite nanoparticles and hematite aggregates. In doing so, the effect of particle size on hematite adsorption capability was assessed. Kinetic and isotherm studies were performed to describe the adsorption process. A comparison of $\mathrm{As}(\mathrm{III})$ and $\mathrm{As}(\mathrm{V})$ adsorption to hematite particles is necessary. Kinetic studies revealed that the initial rate of adsorption of As(III) and As(V) onto hematite nanoparticle and aggregates was fastest within the first $8 \mathrm{~h}$. However, $\mathrm{As}(\mathrm{V})$ can be adsorbed in larger amounts to both hematite nanoparticles and hematite aggregates at equilibrium compared to As(III). The size of the particle is a key factor that can influence the adsorption process. The results from this study revealed that hematite nanoparticles significantly adsorb more As(V) and As(III) at equilibrium in comparison to hematite aggregates. The higher arsenic adsorption by hematite nanoparticles can be explained by the fact that there are a greater number of individual nanoparticles (higher amount of adsorption sites) in the nanoparticle dispersion that can accommodate a high volume of arsenic adsorbing to the surface. Isotherm studies 
showed that hematite nanoparticles has a significantly higher adsorption capacity for both $\mathrm{As}(\mathrm{V})$ and $\mathrm{As}(\mathrm{III})$ than hematite aggregates. The adsorption studies also revealed that aggregation and sedimentation of hematite nanoparticles did occur during arsenic adsorption especially in the period of rapid arsenic adsorption. The aggregation can be explained by the change in the particle surface charge due to arsenic adsorption. These results are important as aggregation can reduce the adsorptive capability of the nanoparticle and slow down the adsorption process. Overall, these findings are important as they further our understanding of the interactions of hematite nanoparticles and hematite aggregates with arsenic in the environment.

\subsection{List of References}

Al-Abed SR, Jegadeesan G, Purandare J, Allen D. Arsenic release from iron rich mineral processing waste: influence of $\mathrm{pH}$ and redox potential. Chemosphere 2006; 66: 775-782.

Auffan M, Rose J, Proux O, Borschneck D, Masion A, Chaurand P, et al. Enhanced Adsorption of Arsenic onto Maghemites Nanoparticles: As(III) as a Probe of the Surface Structure and Heterogeneity. Langmuir 2008; 24: 3215-3222.

Azizian S. Kinetic models of sorption. A theoretical analysis. J Colloid Interface Sci 2004; 276: 47-52.

Banerjee K, Amy GL, Prevost M, Nour S, Jekel M, Gallagher PM, et al. Kinetic and thermodynamic aspects of adsorption of arsenic onto granular ferric hydroxide (GFH). Water Res 2008; 42: 3371-3378.

Carabante I, Grahn M, Holmgren A, Kumpiene J, Hedlund J. Adsorption of As (V) on iron oxide nanoparticle films studied by in situ ATR-FTIR spectroscopy. Colloid Surface A 2009; 346: 106-113.

Cundy AB, Hopkinson L, Whitby RLD. Use of iron-based technologies in contaminated land and groundwater remediation: A review. Sci Total Environ 2008; 400: 42-51.

Dickson D, Liu G, Li C, Tachiev G, Cai Y. Dispersion and stability of bare hematite nanoparticles: Effect of dispersion tools, nanoparticle concentration, humic acid and ionic strength. Sci Total Environ 2012; 419: 170-177. 
Dixit S, Hering JG. Comparison of Arsenic(V) and Arsenic(III) Sorption onto Iron Oxide Minerals: Implications for Arsenic Mobility. Environ Sci Technol 2003; 37:

4182-4189.

Duran C, Ozdes D, Gundogdu A, Senturk HB. Kinetics and Isotherm Analysis of Basic Dyes Adsorption onto Almond Shell (Prunus dulcis) as a Low Cost Adsorbent. J Chem Eng Data 2011; 56: 2136-2147.

Giasuddin ABM, Kanel SR, Choi H. Adsorption of Humic Acid onto Nanoscale Zerovalent Iron and Its Effect on Arsenic Removal. Environ Sci Technol 2007; 41: 2022-2027.

He F, Zhao D. Manipulating the Size and Dispersibility of Zerovalent Iron Nanoparticles by Use of Carboxymethyl Cellulose Stabilizers. Environ Sci Technol 2007; 41: 6216-6221.

He YT, Wan J, Tokunaga T. Kinetic stability of hematite nanoparticles: the effect of particle sizes. J Nanopart Res 2008; 10: 321-332.

Ho YS, McKay G. Pseudo-second order model for sorption processes. Process Biochem 1999; 34: 451-465.

Islam FS, Gault AG, Boothman C, Polya DA, Charnock JM, Chatterjee D, et al. Role of metal-reducing bacteria in arsenic release from Bengal delta sediments. Nature 2004; 430: 68-71.

Jain A, Raven KP, Loeppert RH. Arsenite and Arsenate Adsorption on Ferrihydrite: Surface Charge Reduction and Net OH- Release Stoichiometry. Environ Sci Technol 1999; 33: 1179-1184.

Jeong Y, Fan M, Singh S, Chuang C-L, Saha B, van Leeuwen JH. Evaluation of iron oxide and aluminum oxide as potential arsenic(V) adsorbents. Chem Eng Process 2007; 46: 1030-1039.

Kanel SR, Manning B, Charlet L, Choi H. Removal of arsenic(III) from groundwater by nanoscale zero-valent iron. Environ Sci Technol 2005; 39: 1291-8.

Kanel Sushil R, Manning B, Charlet L, Choi H. Removal of arsenic(III) from groundwater by nanoscale zero-valent iron. Environ. Sci. Technol. 2005; 39: 1291-8.

Ko I, Kim J-Y, Kim K-W. Arsenic speciation and sorption kinetics in the As-hematitehumic acid system. Colloids Surf 2004; 234: 43-50.

Kundu S, Gupta AK. Adsorption characteristics of As(III) from aqueous solution on iron oxide coated cement (IOCC). J Hazard Mater 2007; 142: 97-104. 
Lin J, Wang L. Comparison between linear and non-linear forms of pseudo-first-order and pseudo-second-order adsorption kinetic models for the removal of methylene blue by activated carbon. Front Environ Sci Eng 2009; 3: 320-324.

Mandal BK, Suzuki KT. Arsenic round the world: a review. Talanta 2002; 58: 201-235.

Morgada ME, Levy IK, Salomone V, Farias SS, Lopez G, Litter MI. Arsenic (V) removal with nanoparticulate zerovalent iron: Effect of UV light and humic acids. Catal Today 2009; 143: 261-268.

Ng JC, Wang J, Shraim A. A global health problem caused by arsenic from natural sources. Chemosphere 2003; 52: 1353-1359.

Pedersen HD, Postma D, Jakobsen R. Release of arsenic associated with the reduction and transformation of iron oxides. Geochim Cosmochim 2006; 70: 4116-4129.

Phenrat T, Liu Y, Tilton RD, Lowry GV. Adsorbed Polyelectrolyte Coatings Decrease Fe Nanoparticle Reactivity with TCE in Water: Conceptual Model and Mechanisms. Environ Sci Technol 2009; 43: 1507-1514.

Phenrat T, Saleh N, Sirk K, Tilton RD, Lowry GV. Aggregation and Sedimentation of Aqueous Nanoscale Zerovalent Iron Dispersions. Environ Sci Technol 2007; 41: 284-290.

Raven KP, Jain A, Loeppert RH. Arsenite and Arsenate Adsorption on Ferrihydrite: Kinetics, Equilibrium, and Adsorption Envelopes. Environ Sci Technol 1998; 32: 344-349.

Schwertmann U, Cornell RM. Iron Oxides in the Laboratory: Preparation and Characterization. Weinheim: Wiley, 2000.

Shipley H, Yean S, Kan A, Tomson M. A sorption kinetics model for arsenic adsorption to magnetite nanoparticles. Environ Sci Pollut R 2010a; 17: 1053-1062.

Shipley HJ, Yean S, Kan AT, Tomson MB. Adsorption of arsenic to magnetite nanoparticles: effect of particle concentration, $\mathrm{pH}$, ionic strength, and temperature. Environ Toxicol Chem 2009; 28: 509-515.

Shipley HJ, Yean S, Kan AT, Tomson MB. A sorption kinetics model for arsenic adsorption to magnetite nanoparticles. Environ Sci Pollut R 2010b; 17: 1053-1062.

Skopp J. Derivation of the Freundlich adsorption isotherm from kinetics. J Chem Educ 2009; 86: 1341-1343.

Stipp SLS, Hansen M, Kristensen R, Hochella MF, Bennedsen L, Dideriksen K, et al. Behaviour of $\mathrm{Fe}$-oxides relevant to contaminant uptake in the environment. Chem Geol 2002; 190: 321-337. 
Tufano KJ, Fendorf S. Confounding Impacts of Iron Reduction on Arsenic Retention. Environ Sci Technol 2008; 42: 4777-4783.

Turk T, Alp I, Deveci H. Adsorption of As(V) from water using nanomagnetite. J Environ Eng 2010; 136: 399-404.

Tuutijarvi T, Lu J, Sillanpaa M, Chen G. As(V) adsorption on maghemite nanoparticles. J Hazard Mater 2009; 166: 1415-1420.

Wang C-B, Zhang W-x. Synthesizing Nanoscale Iron Particles for Rapid and Complete Dechlorination of TCE and PCBs. Environ Sci Technol 1997; 31: 2154-2156.

Waychunas GA, Kim CS, Banfield JF. Nanoparticulate iron oxide minerals in soils and sediments: unique properties and contaminant scavenging mechanisms. J Nanopart Res 2005; 7: 409-433.

Zaspalis V, Pagana A, Sklari S. Arsenic removal from contaminated water by iron oxide sorbents and porous ceramic membranes. Desalination 2007; 217: 167-180.

Zhang W-x. Nanoscale Iron Particles for Environmental Remediation: An Overview. J Nanopart Res 2003; 5: 323-332.

Zhang W, Cai Y, Tu C, Ma LQ. Arsenic speciation and distribution in an arsenic hyperaccumulating plant. Sci Total Environ 2002; 300: 167-177.

Zhang W, Singh P, Paling E, Delides S. Arsenic removal from contaminated water by natural iron ores. Miner Eng 2004; 17: 517-524.

Zhang Y, Chen Y, Westerhoff P, Hristovski K, Crittenden JC. Stability of commercial metal oxide nanoparticles in water. Water Res 2008; 42: 2204-2212. 


\section{Chapter IV}

Redox transformation of arsenic in the presence of hematite nanoparticles and hematite aggregates 


\subsection{Abstract}

Toxicity, mobility and bioavailability of arsenic are highly dependent upon its redox state. It is well known that chemical and direct microbial catalyzed redox transformations of arsenic are responsible for arsenic cycling in the environment. However, other environmental factors such as mineral phases must be considered because they can play a significant role in the transformation process. In the environment, iron oxides whether in the nanoparticulate or aggregates form can undergo redox transformations (microbially or photochemically) that can influence or be coupled to the redox transformation of arsenic. Therefore in this work, arsenic redox behavior in the presence of hematite nanoparticles and hematite aggregates were preliminarily quantified. The effect of hematite particle size on the rate and extent of arsenic species transformation and the effect of photochemical processes on the redox transformation of arsenic were investigated. The results showed that As(III) can be oxidized to As(V) and $\mathrm{As}(\mathrm{V})$ can be reduced to $\mathrm{As}(\mathrm{III})$ in the presence of hematite nanoparticles and hematite aggregates. The particle size influences the rate of redox transformation as arsenic transformation occurred more readily and rapidly in the presence of hematite nanoparticles than when interacting with hematite aggregates. Arsenite underwent oxidation in the presence of both hematite nanoparticles and hematite aggregates however reduction of arsenate did minimally. Arsenite oxidation occurred faster in the presence of light than under dark conditions and higher amounts of As(III) was oxidized to $\mathrm{As}(\mathrm{V})$ in the presence of hematite nanoparticles than hematite aggregates. These results improve our understanding of the role of IONP played in arsenic redox transformations in the aquatic environment. 


\subsection{Introduction}

The biogeochemical cycling of arsenic is a complex process and it is crucial to understand the cycling of arsenic in the environment as it will determine its environmental fate. The mobility, bioavailability and toxicity of arsenic are highly dependent upon its oxidation state. In the environment, arsenate and arsenite are the predominant species present with arsenite being more mobile and toxic of the two. The redox behavior is an important factor in determining the form of arsenic species present and this will ultimately determine the extent of toxicity. As(V) and As(III) species in the environment are often subjected to both chemical and microbiological oxidationreduction reactions as well as methylated reactions (Masscheleyn et al., 1991) These reactions can cause arsenic species transformation in which one chemical form of arsenic can be converted to another. The transformation process is important as it can greatly affect arsenic solubility and mobility in the environment making it either more or less bioavailable.

Iron oxides minerals are ubiquitous in the environment and the biogeochemical cycling of iron is strongly intertwined with the biogeochemical cycling of arsenic (Johnston et al., 2011). Arsenate and arsenite are notorious for adsorbing onto iron oxide mineral phases subsequently controlling arsenic mobility and influencing any possible arsenic transformation in the environment. Because of the high presence of iron oxides in nature, arsenic readily interacts with iron oxide minerals primarily though the chemisorption process. From our work (see chapter 3) and others (Raven et al., 1998; Jain et al., 1999; Zaspalis et al., 2007; Shipley et al., 2009), arsenate and arsenite are capable of adsorbing onto iron oxide nanoparticles and iron oxide aggregates. However, 
once adsorbed it is uncertain whether arsenic can remain chemically active to a certain extent (Luther et al., 2005) or can show decreased reactivity due to the formation of IONPs-arsenic complexes that can aggregate and subsequently sediment (Waychunas et al., 2005).

A few studies have investigated arsenic species conversion in the presence of iron oxide minerals such as goethite and ferrihydrite and the observed speciation was explained by microbial or photochemical processes involving the iron oxide minerals. Although these studies did not emphasize the effect of the mineral particle size on arsenic redox transformation, they indicated that arsenic can indeed remain chemically active to a certain extent after adsorption to iron oxides. Therefore, microbial and photochemical processes are important in the environment because they can cause the reduction and/or oxidation of arsenic and iron (Ahmann et al., 1997; Langner et al., 2000; Emett et al., 2001; Bhandari et al., 2011, 2012). Once iron oxide minerals are present, arsenic will adsorb onto these minerals and might undergo surface mediated reduction or oxidation reactions (Yan et al., 2008). Iron oxides can also undergo redox transformation during the arsenic transformation process. Therefore, the redox transformation of both arsenic and iron are coupled.

Many microbes can reduce Fe(III) oxide resulting in dissolution and desorption of arsenic into the aqueous phase (Cummings et al., 1999). Once the reduction and subsequent dissolution of iron oxide particles occur, any adsorbed arsenic can be released into solution. As a result of the reductive dissolution of iron oxides, arsenic can become more mobile and direct aqueous phase arsenic species conversion (reduction of $\mathrm{As}(\mathrm{V})$ to As(III) or the oxidation of As(III) to As(V)) by the same microbes or by other 
microorganism can occur (Langner et al., 2000). Abiotic oxidation of As(III) by dissolved oxygen is a possible mechanism of species transformation although the process is relatively slow (Zhao et al., 2011). Recent studies have shown that photoinduced oxidation of $\mathrm{As}(\mathrm{III})$ to $\mathrm{As}(\mathrm{V})$ can occur in the presence of nanoferrihydrite and goethite (Bhandari et al., 2011, 2012). Bhandari et al. (2011) assumed that in As(III)-ferrihydrite system, the oxidation of As(III) occurred on the ferrihydrite surface until the surface becomes saturated with $\mathrm{As}(\mathrm{V})$. Bhandari et al. (2011) also proposed that aqueous Fe(II) was formed from the reduction of Fe(III) associated with the iron oxide phases and the As(III) oxidation is similar under oxic and anoxic conditions. In the As(III)-goethite system, Bhandari et al. (2012) cannot determine whether the oxidation of As(III) occurred on the goethite surface however higher amounts of As(III) oxidation is observed under oxic conditions than anoxic conditions. So far, information regarding photoinduced arsenic speciation in the presence of iron oxide is limited in the literature. Furthermore, no prior studies have investigated the photoinduced oxidation of As(III) in the presence of hematite nanoparticles or hematite aggregates. Therefore, it is necessary to investigate arsenic species transformation in the presence of hematite mineral phase. Moreover, studies in the literature lack information on whether the particle size might influence the rate and extent of arsenic speciation since the reactivity of iron oxides are highly dependent upon its particle size.

The objective of the study was aimed to determine the effect of hematite particles on the redox transformation of $\mathrm{As}(\mathrm{V})$ and $\mathrm{As}(\mathrm{III})$. To our best knowledge, no prior study has clarified if the hematite particle size can influence arsenic species conversion and if so how does the particle size influence the rate and extent of transformation. The focus of 
the study was to determine and quantify the key transformation process of arsenic in the presence of hematite nanoparticles and hematite aggregates. The study investigated the effect of particle size on the rate and extent of speciation. In addition, the effect of photochemical processes on the redox transformation of arsenic during its interaction with hematite nanoparticles and hematite aggregates were determined. The results obtained will improve our understanding of the behavior of hematite nanoparticles and hematite aggregates with regard to arsenic redox transformation in the environment.

\subsection{Procedures and Methods}

\subsubsection{Materials and Chemicals}

Commercial iron oxide nanoparticles $\left(\alpha-\mathrm{Fe}_{2} \mathrm{O}_{3}, 98 \%\right.$ purity and $50 \mathrm{~m}^{2} / \mathrm{g}$ specific surface area) were purchased from Nanostructured and Amorphous Materials (Houston, Texas). Stock solutions of $1000 \mathrm{mg} / \mathrm{L}$ As (III) and As (V) were prepared by dissolving sodium (meta)arsenite $\left(\mathrm{NaAsO}_{2}, 98 \%\right.$ purity) and sodium arsenate dibasic heptahydrate $\left(\mathrm{Na}_{2} \mathrm{HAsO}_{4} .7 \mathrm{H}_{2} \mathrm{O}, 99 \%\right.$ purity), respectively in nanopure $18.2 \mathrm{M} \Omega$ water produced from a nanopure diamond lab water system (Barnstead Thermolyne Corporation, Dubuque, IA). The arsenic standards were reagent grade and obtained from Aldrich Chemical Company (Milwaukee, WI.). Sodium azide was purchased from Fisher Scientific (Fairlawn, NJ, USA).

\subsubsection{Instrumentation}

Iron oxide nanoparticles were dispersed by probe ultrasonication using a Fisher Scientific sonic dismembrator model 100 (Pittsburg, PA) to prepare nanosize hematite particle. The IONPs were dispersed by vortex using a Fisher Scientific touch mixer model 232 (Pittsburgh, PA) to prepare micrometer size particles. Speciation and 
quantitative determination of arsenic was conducted using high performance liquid chromatography -inductively coupled plasma-Mass Spectrometry (HPLC-ICP/MS) (Perkin Elmer). Prior to arsenic speciation analysis, samples were centrifuged with a Fisher Marathon 21000R centrifuge (Needham Heights, MA) to remove the hematite nanoparticles and aggregates from the aqueous phase.

\subsubsection{Experimental Procedures}

\subsubsection{Arsenic Speciation in the presence of hematite nanoparticles}

Arsenic speciation studies were performed for both $\mathrm{As}(\mathrm{III})$ and $\mathrm{As}(\mathrm{V})$ in the presence of hematite nanoparticles. Speciation studies were performed by mixing 10 $\mathrm{mg} / \mathrm{L}$ hematite nanoparticles with $200 \mu \mathrm{g} / \mathrm{L}$ of $\mathrm{As}(\mathrm{III})$ or $\mathrm{As}(\mathrm{V})$ in $50 \mathrm{~mL}$ polypropylene centrifuge tubes. IONPs were dispersed using probe ultrasonication for 20 minutes at power level 6 to generate nanosize hematite particles following a procedure previosly developed (Dickson et al., 2012). All samples were prepared in nanopure $18.2 \mathrm{M} \Omega$ water and the $\mathrm{pH}$ varied in the range of $7.2-8$ as the suspensions were not buffered. Samples were agitated by pulsing with probe ultrasonicator every 2-4 hrs. Aliquots of samples $(2 \mathrm{~mL})$ were collected at definite time intervals $(0-168 \mathrm{~h})$ and arsenic speciation and quantification in solution was monitored as a function of time. The aliquots were centrifuged at 10,000 rpm for 60 minutes which was sufficient to separate IONPs from aqueous phase (see Chapter 3). Arsenic speciation was monitored using HPLC-ICP/MS by analyzing the supernatant for As(III) and As(V) concentrations. For the separation of As(III) and As(V), a Hamilton PRPX-200 cation exchange column ( $250 \times 4.1 \mathrm{~mm}$ in dimension and 10 micron particle size $)$ was employed with a mobile phase $(0.05 \%$ formic acid $)$ flow rate of $1 \mathrm{~mL} \cdot \mathrm{min}^{-1}$ and the 
sample injection volume was $50 \mu \mathrm{L}$. As(III) and As(V) controls $(200 \mu \mathrm{g} / \mathrm{L})$ were prepared except without hematite nanoparticles added. The control was exposed to similar experimental conditions as the As-hematite nanoparticle samples. Arsenic specation in the control was monitored over a period of $168 \mathrm{~h}$.

\subsubsection{Arsenic speciation in the presence of hematite aggregates}

Arsenic speciation experiments with hematite aggregates were performed with similar procedures as described in section 4.2.3.1 with the exception that hematite particles were dispersed using vortex for 20 minutes at level 10 . The vortex dispersion method generates particles in the micrometer size range (Dickson et al., 2012). The samples were agitated by shaking on an orbital shaker at $250 \mathrm{rpm}$ for the entire experimental time. Arsenic speciation was monitored using HPLC-ICP/MS.

\subsubsection{Photochemical effect on the species transformation of arsenite and arsenate} in the presence of hematite nanoparticles and hematite aggregates

As(III)-hematite nanoparticles suspensions and As(III)-hematite aggregate suspensions were investigated for the effect of light on arsenite oxidation. For the As(III)hematite nanoparticle suspensions, two set of samples containing As(III) $(250 \mu \mathrm{g} / \mathrm{L})$ and $10 \mathrm{mg} / \mathrm{L}$ of hematite nanoparticles were prepared similarly to experiment 4.2.3.1. For the As(III)-hematite aggregate suspensions, two set of samples containing As(III) $(200 \mu \mathrm{g} / \mathrm{L})$ and $10 \mathrm{mg} / \mathrm{L}$ of hematite nanoparticles were prepared similarly to experiment 4.2.3.1. One set of sample was exposed to room light and the other set was kept in the dark. Samples ( $2 \mathrm{~mL}$ aliquots) from each trial were removed in triplicates at 24,48 , and $168 \mathrm{~h}$ and measured for As(III) and As(V) concentrations using HPLC-ICP/MS. 
To determine if microbes were assisting the speciation process during the photochemical experiment, sodium azide was used to inhibit microbial redox transformation. Sodium azide $(0.02 \%)$, a salt, was added to the nanoparticle suspension $(10 \mathrm{mg} / \mathrm{L})$ and monitored for hematite nanoparticles stability. Aliquots of $2 \mathrm{~mL}$ were removed from the suspension in triplicate at $0,1,2,4,8,24,48,72,96$ and $144 \mathrm{~h}$. The hematite nanoparticle suspensions were analyzed for iron concentrations using GFAAS to monitor the sedimentation of IONPs over time. Before iron analysis, IONPs suspension underwent acid digestion in $50 \%$ nitric acid at $95 \pm 5^{\circ} \mathrm{C}$ for 20 minutes using heating block (Environmental Express).

$\operatorname{As}(\mathrm{V})$-hematite nanoparticle suspensions and $\mathrm{As}(\mathrm{V})$-hematite aggregate suspensions were investigated for the effect of light on arsenate reduction. Two set of samples containing $\mathrm{As}(\mathrm{V})(250 \mu \mathrm{g} / \mathrm{L})$ and $10 \mathrm{mg} / \mathrm{L}$ of hematite nanoparticles were prepared similarly to experiment 4.2.3.1. For the $\mathrm{As}(\mathrm{V})$-hematite aggregate suspensions, two set of samples containing $\mathrm{As}(\mathrm{V})(200 \mu \mathrm{g} / \mathrm{L})$ and $10 \mathrm{mg} / \mathrm{L}$ of hematite nanoparticles were prepared similarly to experiment 4.2.3.1. One set of sample was exposed to room light and the other set was in the dark. Samples ( $2 \mathrm{~mL}$ aliquots) were removed in triplicate at 24, 48 and 168h. Arsenic speciation was monitored using HPLC-ICP/MS.

\subsection{Results and Discussion}

\subsubsection{The effect of hematite particle size on arsenite and arsenate redox transformation}

Experiments were performed to determine whether As(III) can be oxidized to $\operatorname{As}(\mathrm{V})$ and $\mathrm{As}(\mathrm{V})$ can be reduced to $\mathrm{As}(\mathrm{III})$ in the presence of hematite nanoparticles and hematite aggregates. These samples contained arsenic with hematite nanoparticles or 
hematite aggregates and no other factors were added or controlled. Figures 4.1a and 4.2a showed that both $\mathrm{As}(\mathrm{III})$ and $\mathrm{As}(\mathrm{V})$ can undergo redox transformation in the presence of hematite nanoparticles.

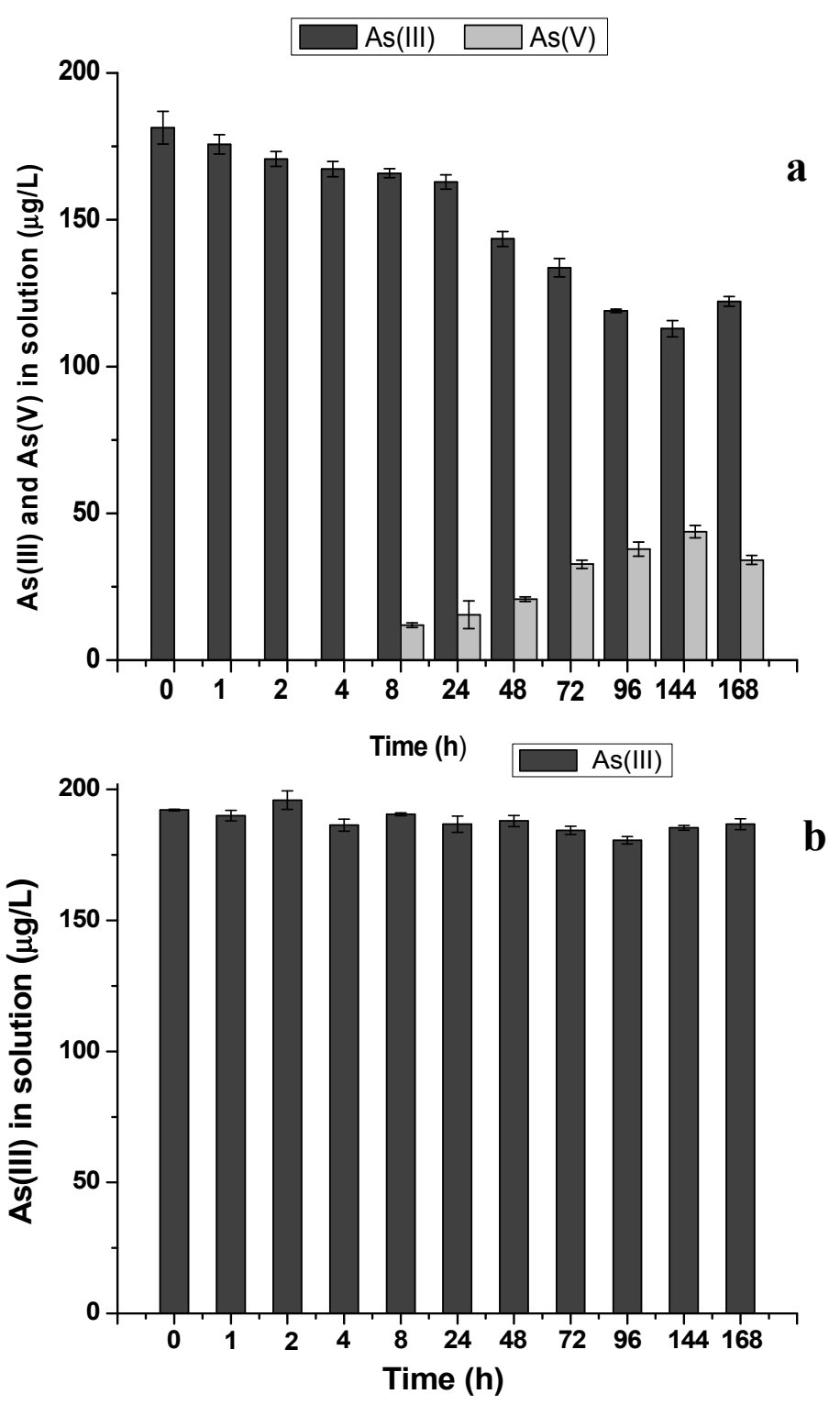

Figure 4. 1Arsenite speciation studies over a contact time of 168h. (a) As(III) $(200 \mu \mathrm{g} / \mathrm{L})$ with $10 \mathrm{mg} / \mathrm{L}$ hematite nanoparticles under room light at $20^{\circ} \mathrm{C}$ and (b) $\mathrm{As}(\mathrm{III})$ control $\left(200 \mu \mathrm{g} / \mathrm{L}\right.$ of $\mathrm{As}(\mathrm{III})$ in deionized water under room light at $\left.20^{\circ} \mathrm{C}\right)$ 

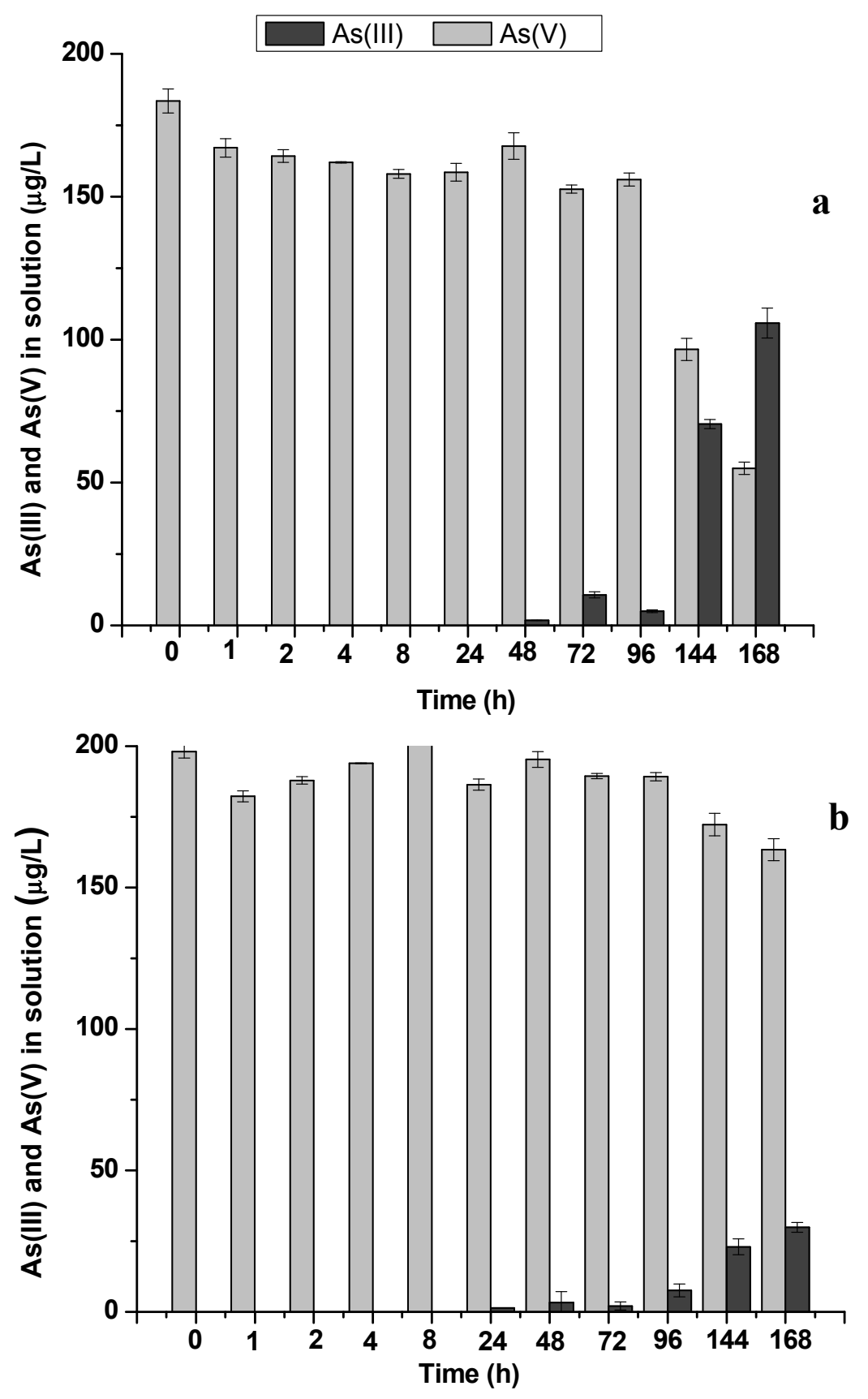

Figure 4. 2 Arsenate speciation studies over a contact time of 168h. (a) As(V) (200 $\mu \mathrm{g} / \mathrm{L})$ with $10 \mathrm{mg} / \mathrm{L}$ hematite nanoparticles under room light at $20^{\circ} \mathrm{C}$ and (b) $\mathrm{As}(\mathrm{V})$ control 
In As(III)-hematite nanoparticles samples, As(III) was oxidized to As(V) starting at $\mathrm{t}_{8 \mathrm{~h}}$ with As(III) oxidation increasing overtime as evidenced by the increasing amounts of As(V) in solution (Fig. 4.1a). Approximately $21-27 \%$ of the As(III) was oxidized to As(V) over the course of $168 \mathrm{~h}$. However, no As(III) oxidation was observed in the control throughout the entire experimental time (Fig. 4.1b). In As(V)-hematite nanoparticles samples, $\operatorname{As}(\mathrm{V})$ was reduced to $\mathrm{As}(\mathrm{III})$ at $\mathrm{t}_{24 \mathrm{~h}}$ with increasing reduction overtime as evidenced by the increasing amounts of As(III) in solution (Fig. 4.2a). In the control, $\mathrm{As}(\mathrm{V})$ reduction was also observed but at $\mathrm{t}_{24 \mathrm{~h}}$ and increasing slightly with time (Fig. 4.2b). A comparison between $\mathrm{As}(\mathrm{V})$-hematite nanoparticle sample and $\mathrm{As}(\mathrm{V})$ control showed that at $\mathrm{t}_{168 \mathrm{~h}} 65 \%$ of $\mathrm{As}(\mathrm{V})$ was reduced to $\mathrm{As}(\mathrm{III})$ in the sample compared to only $15 \%$ in the control. At this point, it is not clear whether or not the nanoparticle adsorbed the newly converted species but the adsorption of these species are possible as from the previous adsorption experiments (see chapter 3) hematite nanoparticles were capable of adsorbing both $\mathrm{As}(\mathrm{III})$ and $\mathrm{As}(\mathrm{V})$.

To gain insight on how the size of the particle influences arsenic species conversion, arsenic speciation was performed in the presence of hematite aggregates. In As(III)-hematite aggregates samples, no oxidation of As(III) to As(V) was observed during the course of the experiment (Figure 4.3a). Control studies also indicated no As(III) oxidation over the course of 168h (Figure 4.3b). However, in As(V)-hematite aggregates samples, $\mathrm{As}(\mathrm{V})$ was reduced to $\mathrm{As}(\mathrm{III})$ at $\mathrm{t}_{72 \mathrm{~h}}$ with slight increase in reduction through $\mathrm{t}_{168 \mathrm{~h}}$ (Fig. 4.4a). As(V) reduction was also observed in the control but only at $\mathrm{t}_{168 \mathrm{~h}}$ (Figure 4.4b). A comparison of the As(V)-hematite aggregate sample and the $\mathrm{As}(\mathrm{V})$ 
control showed that only $6 \%$ As(III) was found in aqueous phase in the As(V)-hematite aggregates sample compared to only $17 \%$ in the control.
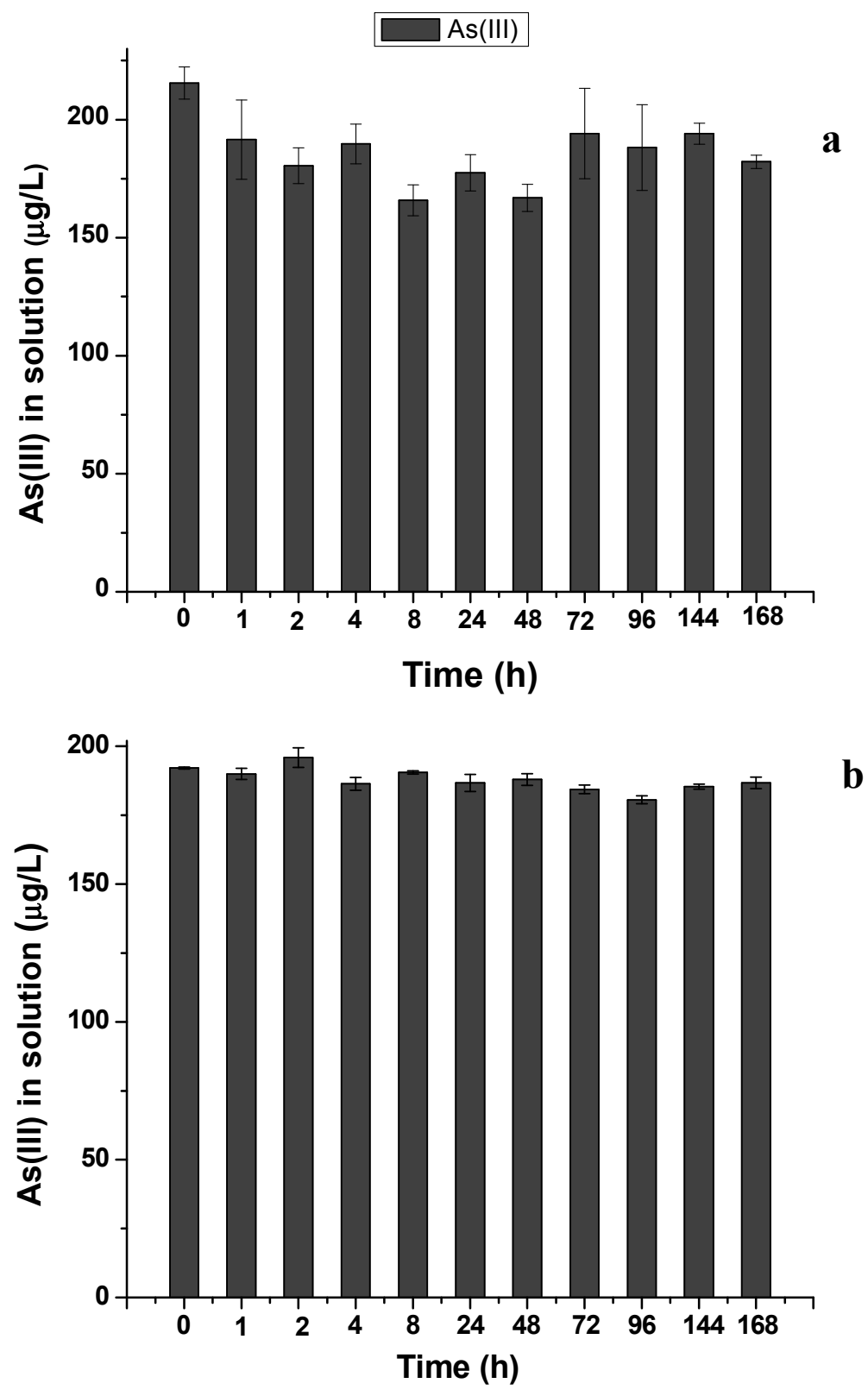

Figure 4. 3Arsenite speciation studies over a contact time of 168h. (a) As(III) (200 $\mu \mathrm{g} / \mathrm{L})$ with $10 \mathrm{mg} / \mathrm{L}$ hematite aggregates under room light at $20^{\circ} \mathrm{C}$ and (b) $\mathrm{As}(\mathrm{III})$ control 


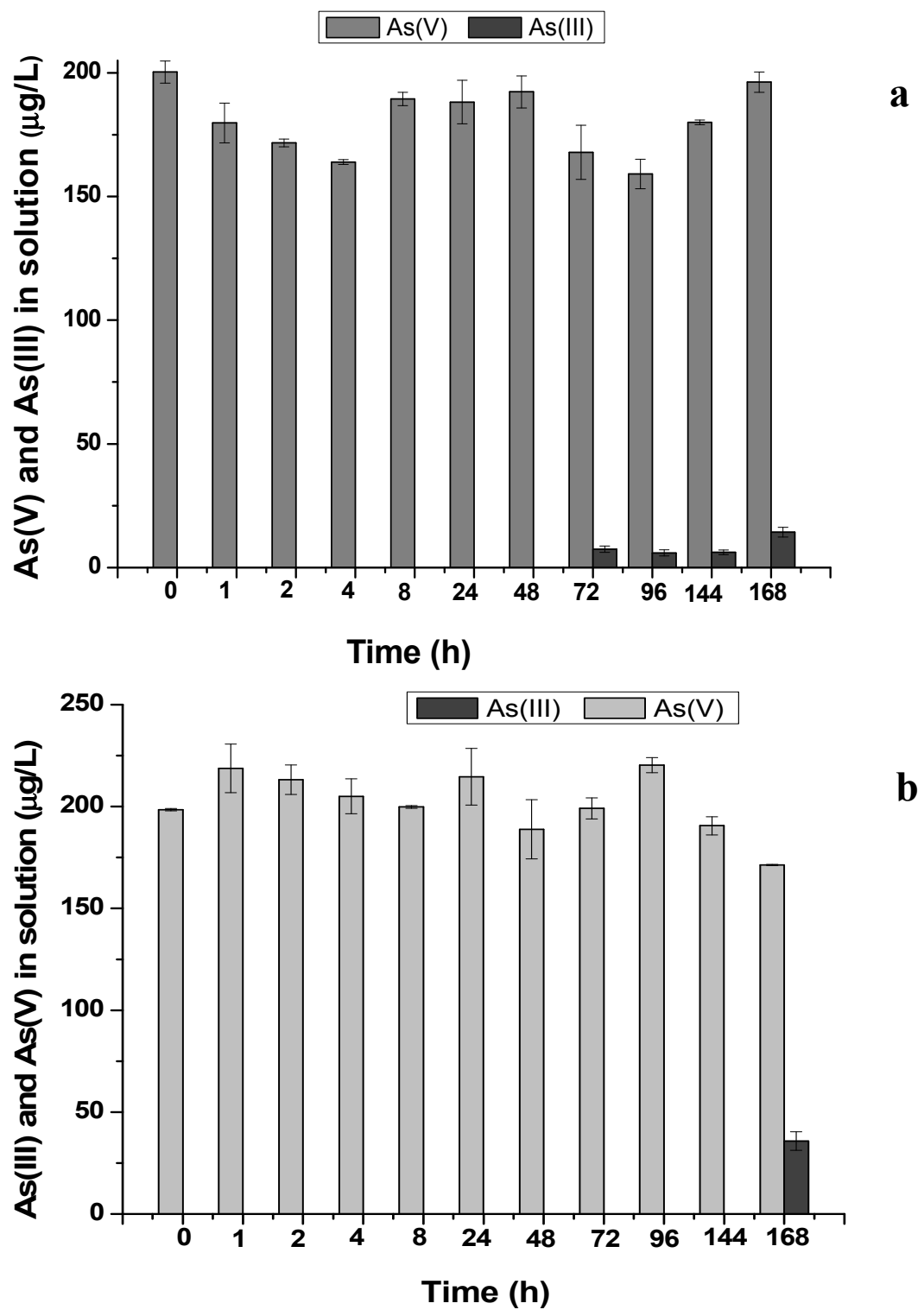

Figure 4. 4 Arsenate speciation studies over a contact time of 168h. (a) As(V) $(200 \mu \mathrm{g} / \mathrm{L})$ with $10 \mathrm{mg} / \mathrm{L}$ hematite aggregates under room light at $20^{\circ} \mathrm{C}$ and (b) $\mathrm{As}(\mathrm{V})$ control 
The increase in the newly converted arsenic species in the aqueous phase is a good indication that arsenic redox transformation does occur in the presence of hematite nanoparticles and hematite aggregates and the transformation is increasing with time. The rate of $\mathrm{As}(\mathrm{V})$ and $\mathrm{As}(\mathrm{III})$ species conversion was faster in the presence of hematite nanoparticles than hematite aggregates ( $\mathrm{z}$ test, $\mathrm{p}<0.05)$. Moreover, $\mathrm{As}(\mathrm{V})$ speciation was observed at $\mathrm{t}_{24 \mathrm{~h}}$ for the hematite nanoparticles in comparison to $\mathrm{t}_{72 \mathrm{~h}}$ for hematite aggregates. In addition, As(III) oxidation was observed in the presence of hematite nanoparticle starting at $\mathrm{t}_{8 \mathrm{~h}}$ while no oxidation was observed in the aggregated dispersion throughout the entire experimental time (168h). Since the amount of $\mathrm{As}(\mathrm{V}) / \mathrm{As}(\mathrm{III})$ that is reduced/oxidized in the presence of hematite nanoparticles is greater than the control ( $\mathrm{z}$ test, $\mathrm{p}<0.05)$, factors relating to the iron oxide is possibly causing $\mathrm{As}(\mathrm{V})$ reduction or As(III) oxidation. Furthermore, a higher percentage of As(III) or As(V) was transformed in the presence of hematite nanoparticles than hematite aggregates, suggesting that As(III) and $\mathrm{As}(\mathrm{V})$ more readily undergoes redox transformation in the presence of hematite nanoparticles than hematite aggregates.

The factors that are fully responsible for species transformation and the mechanisms of speciation are not yet determined but the particle size is likely one of the major influences on how fast species transformation occurs. Small particles have high surface area that can accommodate higher amounts of arsenic for adsorption. Any surface mediated arsenic transformation would therefore occur faster and in higher amounts on the nanoparticle's surface than on the hematite aggregates' surfaces. 


\subsubsection{The effect of light on arsenite redox transformation in the presence of iron oxide nanoparticles and iron oxide aggregates.}

So far arsenic transformation was observed in the presence of hematite nanoparticles and aggregates when no additional factors were added and the samples were exposed to light. Based on these experimental conditions, photochemical processes might be one of the factors that are possibly causing arsenic redox transformation. Therefore experiments were performed where arsenic speciation was monitored when arsenic was exposed to hematite nanoparticles and hematite aggregates under light and dark conditions. Figure 4.5 shows the speciation of As(III) in the presence of hematite nanoparticles under light and dark conditions for 24,48 and $168 \mathrm{~h}$.

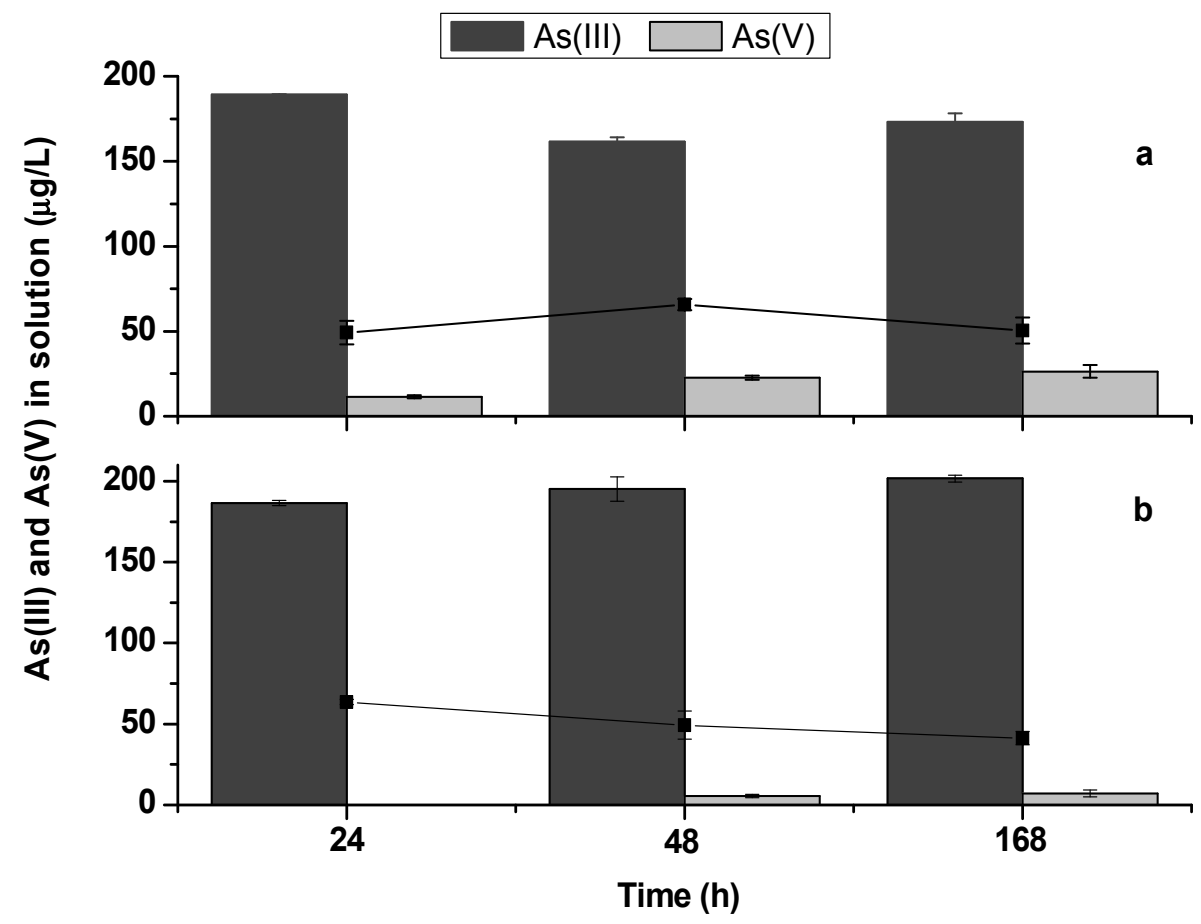

Figure 4. $5 \mathrm{As}(\mathrm{III})(250 \mu \mathrm{g} / \mathrm{L})$ speciation studies in the presence of hematite nanoparticles $(10 \mathrm{mg} / \mathrm{L})$ in (a) light (b) dark. The columns represent arsenic in solution $(\mu \mathrm{g} / \mathrm{L})$. The line represents the amount of arsenic is adsorbed $(\mu \mathrm{g} / \mathrm{L})$ onto the hematite nanoparticles. 
As(III) was oxidized to As(V) under both light and dark conditions. However, higher amounts of As(III) oxidation were observed under light with species transformation increasing slightly with time (Figure. 4.5a). There was minimal As(III) oxidation in the dark and oxidation occurred slower than when exposed to light (Figure. 4.5b) (z test, p < 0.05). However, light did not affect the amount of As(III) that was adsorbed as similar amounts of As(III) was adsorbed onto hematite nanoparticles under light and dark conditions (Figure 4.5 line) ( $\mathrm{z}$ test, $\mathrm{p}>0.05)$.

Figure 4.6 shows the speciation of As(III) in the presence of hematite aggregates when exposed to light and dark conditions for 24,48 , and $168 \mathrm{~h}$. As(III) oxidation was observed when the As(III)-hematite aggregates suspension was exposed to light (Figure 4.6a), while no speciation was observed in the dark (Figure 4.6b). Control experiments were performed where As(III) in the absence of hematite nanoparticles and hematite aggregates were exposed to light and dark conditions similar to the As(III)-hematite samples. The control showed no oxidation of As(III) either in the light or in the dark over the course of $168 \mathrm{~h}$ (Figures 4.7 and 4.8). These results indicate that photochemical reactions might play a role in As(III) oxidation. Arsenite oxidation in the presence of both hematite nanoparticles and hematite aggregates occurred faster in the presence of light than in the dark. However, more As(III) was oxidized to As(V) in the presence of hematite nanoparticles than hematite aggregates over the course of $168 \mathrm{~h}$ as indicated by the higher amounts of $\mathrm{As}(\mathrm{V})$ in the aqueous phase ( $\mathrm{z}$ test, $\mathrm{p}<0.05)$. 


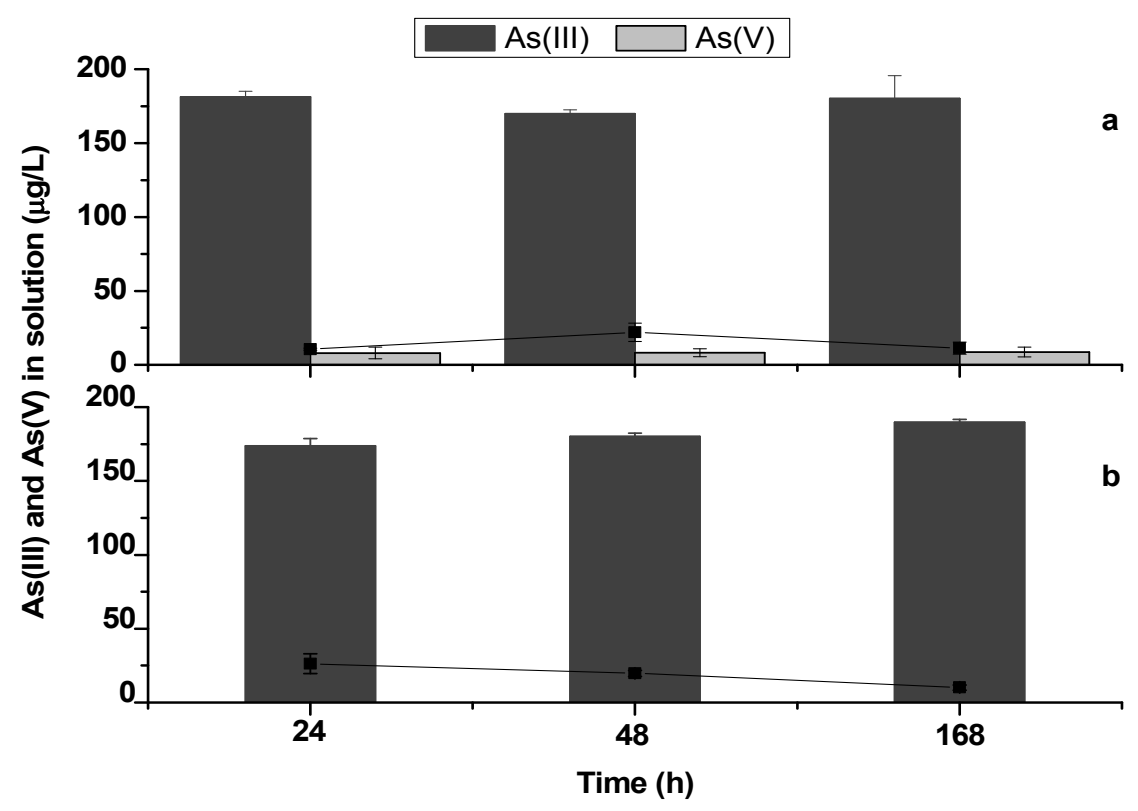

Figure 4. $6 \mathrm{As}(\mathrm{III})(200 \mu \mathrm{g} / \mathrm{L})$ speciation studies in the presence of hematite aggregates $(10 \mathrm{mg} / \mathrm{L})$ in (a) light (b) dark. The columns represent arsenic in solution $(\mu \mathrm{g} / \mathrm{L})$ and the line represents the amount of arsenic $(\mu \mathrm{g} / \mathrm{L})$ was adsorbed onto the hematite aggregates.

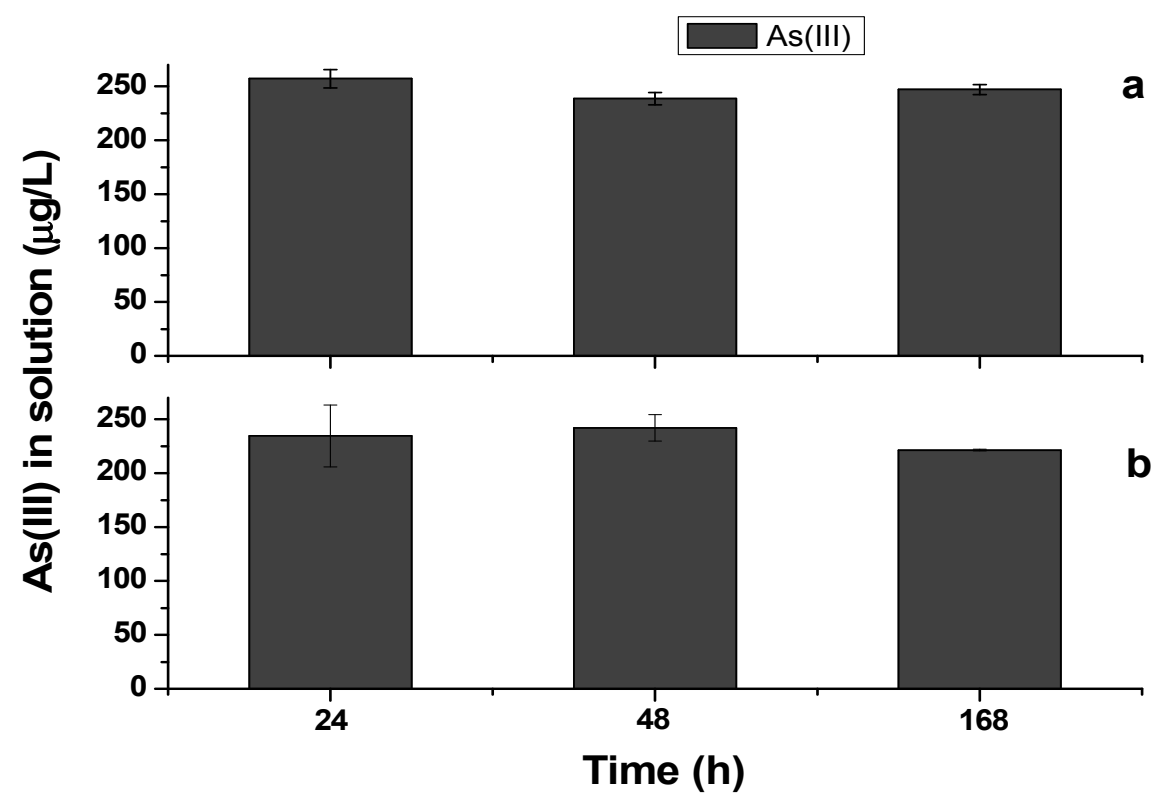

Figure 4. $7 \mathrm{As}(\mathrm{IIII})$ control $(250 \mu \mathrm{g} / \mathrm{L})$ performed during the As(III)-hematite nanoparticles speciation experiment (a) light (b) dark 


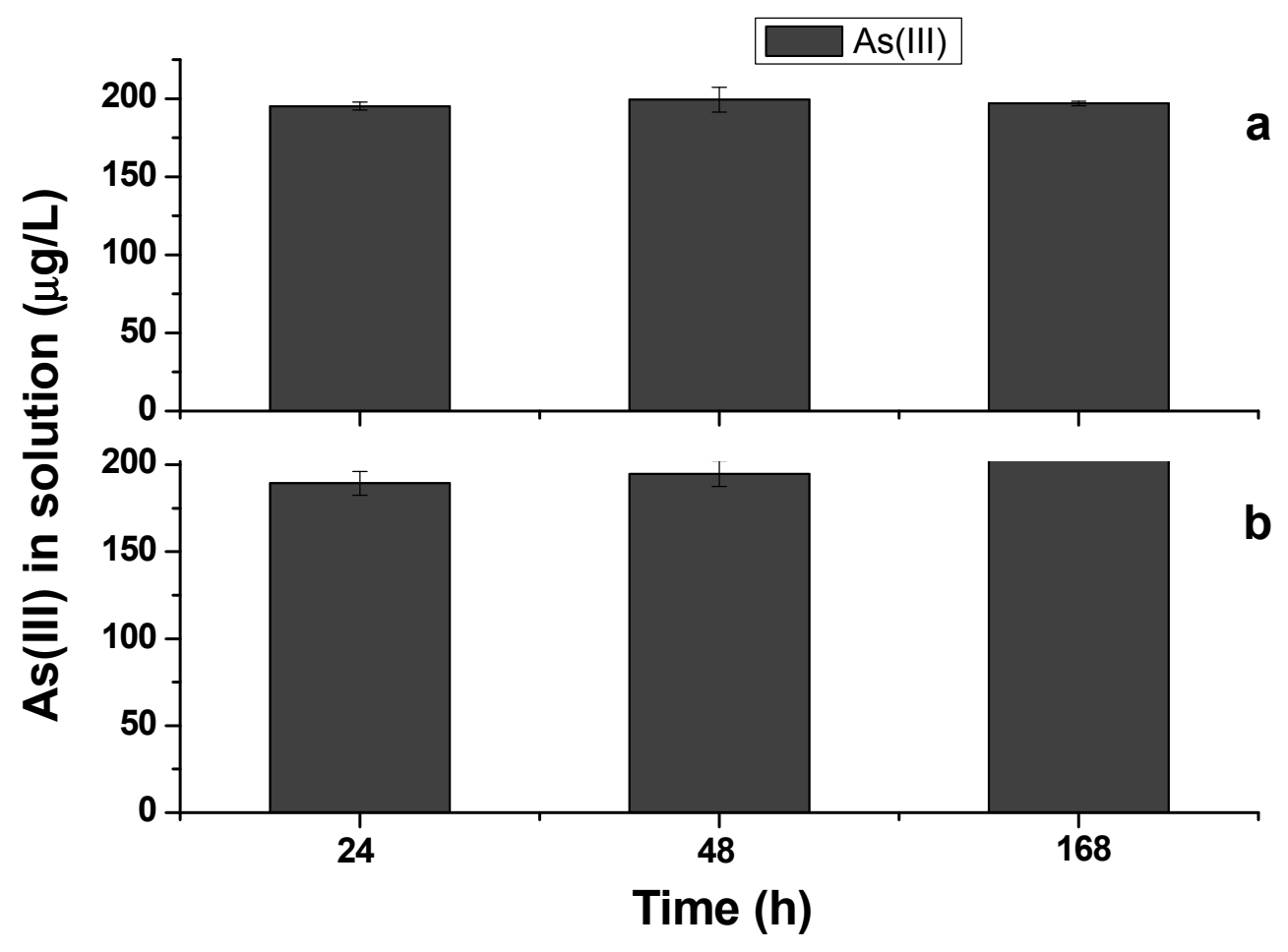

Figure 4. $8 \mathrm{As}(\mathrm{III})$ control $(200 \mu \mathrm{g} / \mathrm{L})$ performed during the As(III)-hematite aggregates speciation experiment (a) light (b) dark.

It could be concluded from these results that the rate of photochemical oxidation of As(III) is dependent upon the size of the hematite particle. Nanoparticles can have optical and electronic properties that are different from the aggregated or bulk form (Altman et al., 2001; Sosa et al., 2003). It is known that nanoparticles have high surface area which can ultimately increase the photocatalytic activity of the hematite particle (Tang et al., 2004). From the results, higher As(III) oxidation transformation efficiency was observed in the presence of the nanoparticles than the aggregates. Since the amount of As(III) oxidized is particle size dependent, it is presumed that As(III) oxidation occurs 
on the surface of the hematite particles (nanoparticle or aggregate). As mentioned before, nanoparticles are more reactive than micrometer sized particles indicated by the higher amounts of arsenic that was adsorbed onto the nanoparticle surface compared to the aggregates (Figures. 4.5 and 4.6 lines). Since more As(III) was adsorbed onto the nanoparticle surface, then higher amounts of As(III) is subjected to surface mediated oxidation possibly explaining why As(III) oxidation was greatest in the presence of hematite nanoparticles.

The mechanism of the photoinduced redox transformation of arsenic, to the best of our knowledge, has not been extensively studied. Figure 4.9 shows a schematics of the proposed pathway of the surface mediated oxidation of As(III) in the presence of hematite particles modified from Stumm and Morgan (Stumm et al., 1996). It is assumed that after As(III) adsorption to the hematite surface and upon exposure to light, the insoluble Fe(III) gets reduced to the soluble Fe(II) and the adsorbed As(III) is oxidized to $\mathrm{As}(\mathrm{V})$. Both $\mathrm{Fe}(\mathrm{II})$ and $\mathrm{As}(\mathrm{V})$ are then released into the aqueous phase. This assumption is formed on the basis that many metal oxides can behave as semiconductors absorbing light energy directly. After light is absorbed, the excited state produce a charge separation $\left(\mathrm{e}^{-}\right.$and holes $\left.\mathrm{h}^{+}\right)$in the energy band gap which can form reducing and oxidizing sites at the particle surface (Zou et al., 2001). The electrons are reducing and can reduce Fe(III) to $\mathrm{Fe}(\mathrm{II})$ and the holes are oxidizing where it can oxidize $\mathrm{As}(\mathrm{III})$ to $\mathrm{As}(\mathrm{V})$. 


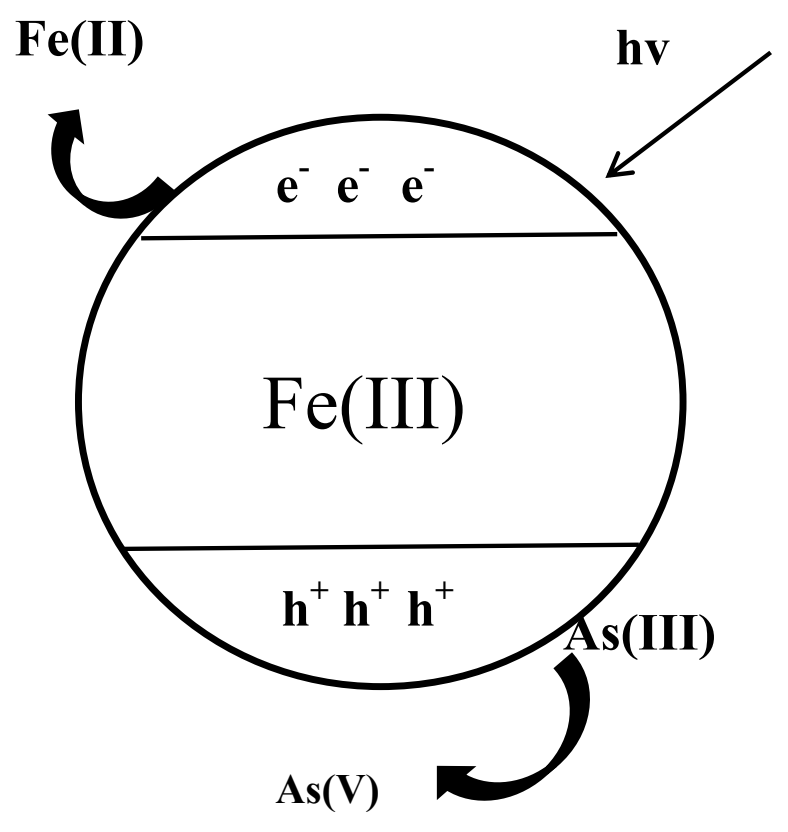

Figure 4. 9 Proposed pathway for surface mediated As(III) oxidation in the presence of $\alpha$ $\mathrm{Fe}_{2} \mathrm{O}_{3}$ (hematite). After light absorption, a strong reducing electron and a strong oxidizing hole are formed. $\mathrm{Fe}(\mathrm{III})$ is reduced to $\mathrm{Fe}(\mathrm{II})$ by the reducing electron and $\mathrm{As}(\mathrm{III})$ is oxidized to $\mathrm{As}(\mathrm{V})$ via the oxidizing hole, $\mathrm{h}^{+}$. Both $\mathrm{Fe}(\mathrm{II})$ and $\mathrm{As}(\mathrm{V})$ are then released into solution.

The proposed reactions below can explain and summarize the mechanisms of the photochemical redox transformation (Stumm et al., 1996; Motamedi et al., 2003;

Baumanis et al., 2011).

$$
\begin{array}{ll}
\alpha-\mathrm{Fe}_{2} \mathrm{O}_{3}+\mathrm{hv} \rightarrow \mathrm{e}_{\mathrm{CB}}^{-}+\mathrm{h}_{\mathrm{VB}}^{+} & 1 \\
\mathrm{Fe}(\mathrm{III})+\mathrm{e}_{\mathrm{CB}}^{-} \rightarrow \mathrm{Fe}(\mathrm{II}) & 2 \\
\mathrm{As}(\mathrm{III})+\mathrm{h}_{\mathrm{VB}}^{+} \rightarrow \mathrm{As}(\mathrm{IV}) & 3
\end{array}
$$


$\mathrm{As}(\mathrm{IV})+\mathrm{O}_{2} \rightarrow \mathrm{As}(\mathrm{V})+\mathrm{O}_{2}{ }^{-}$

$\mathrm{As}(\mathrm{IV})+\mathrm{As}(\mathrm{IV}) \rightarrow \mathrm{As}(\mathrm{III})+\mathrm{As}(\mathrm{V}) \quad 5$

Overall, photochemical processes play an important role in the biogeochemical cycling of iron and arsenic by influencing their redox processes (Equations 1-5). To fully comprehend the effect of light on As(III) oxidation in the presence of iron oxides nanoparticles, future studies need to be performed to confirm the proposed mechanism of the redox transformation (see Chapter 5).

\subsubsection{The effect of light on arsenate redox transformation in the presence of iron oxide nanoparticles and iron oxide aggregates}

Figure 4.10 shows the speciation of $\mathrm{As}(\mathrm{V})$ in the presence of hematite nanoparticles under light and dark conditions for 24,48 and 168h. There was minimal $\operatorname{As}(\mathrm{V})$ reduction in the presence and absence of light. However, the amount of $\operatorname{As}(\mathrm{V})$ reduced at $\mathrm{t} 168 \mathrm{~h}$ under light conditions was similar to the amount of $\mathrm{As}(\mathrm{V})$ reduced under dark conditions. In addition, arsenate species conversion was also investigated in the presence of hematite aggregates, however, there was no observation of $\operatorname{As}(\mathrm{V})$ reduction either in the presence or absence of light (Figure 4.11). Control studies also showed minimal to no $\mathrm{As}(\mathrm{V})$ reduction in the presence or absence of light (Figure 4.12 and 4.13). These results indicate that photochemical processes are not responsible for $\operatorname{As}(\mathrm{V})$ reduction in the presence of hematite particles. Based on the proposed mechanisms, it is difficult for $\mathrm{As}(\mathrm{V})$ to be reduced photochemically. The $\mathrm{e}^{-}$that is generated upon the absorption of light, is used to reduce Fe(III) to Fe(II). The $\mathrm{h}^{+}$usually generates oxidizing regions on the surface of the particles and therefore cannot reduce $\operatorname{As}(\mathrm{V})$. 


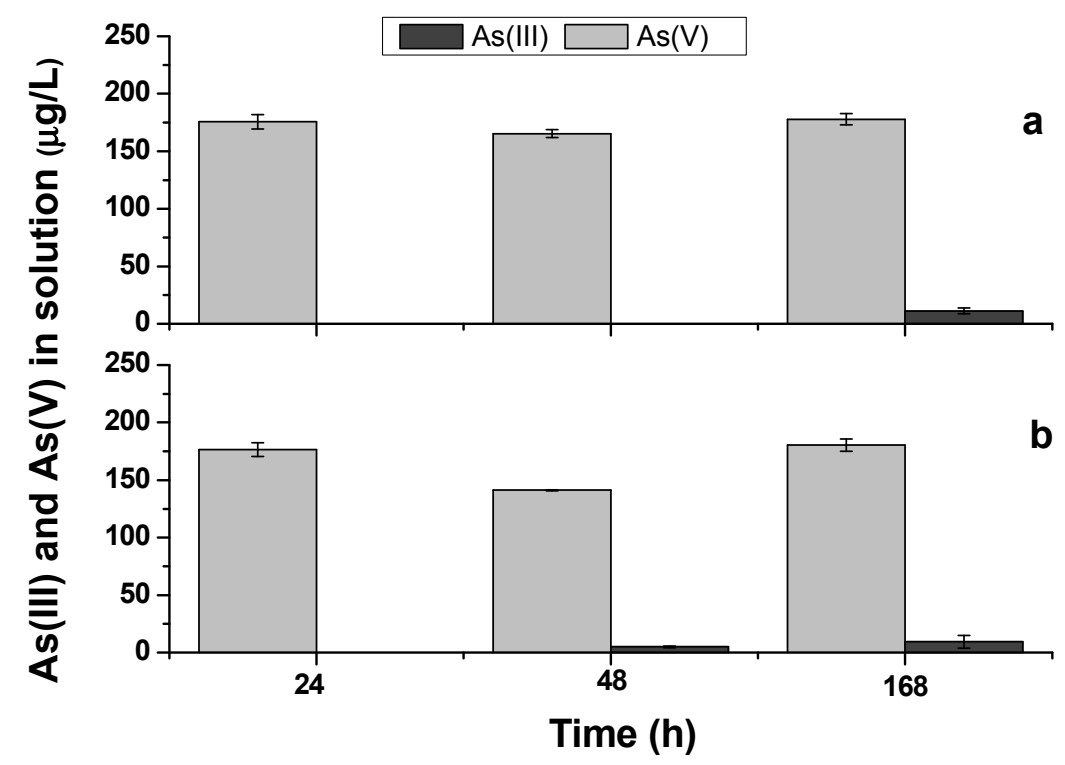

Figure 4. $10 \mathrm{As}(\mathrm{V})(250 \mu \mathrm{g} / \mathrm{L})$ speciation studies in the presence of hematite nanoparticles in (a) light (b) dark. The columns represent arsenic in solution $(\mu \mathrm{g} / \mathrm{L})$

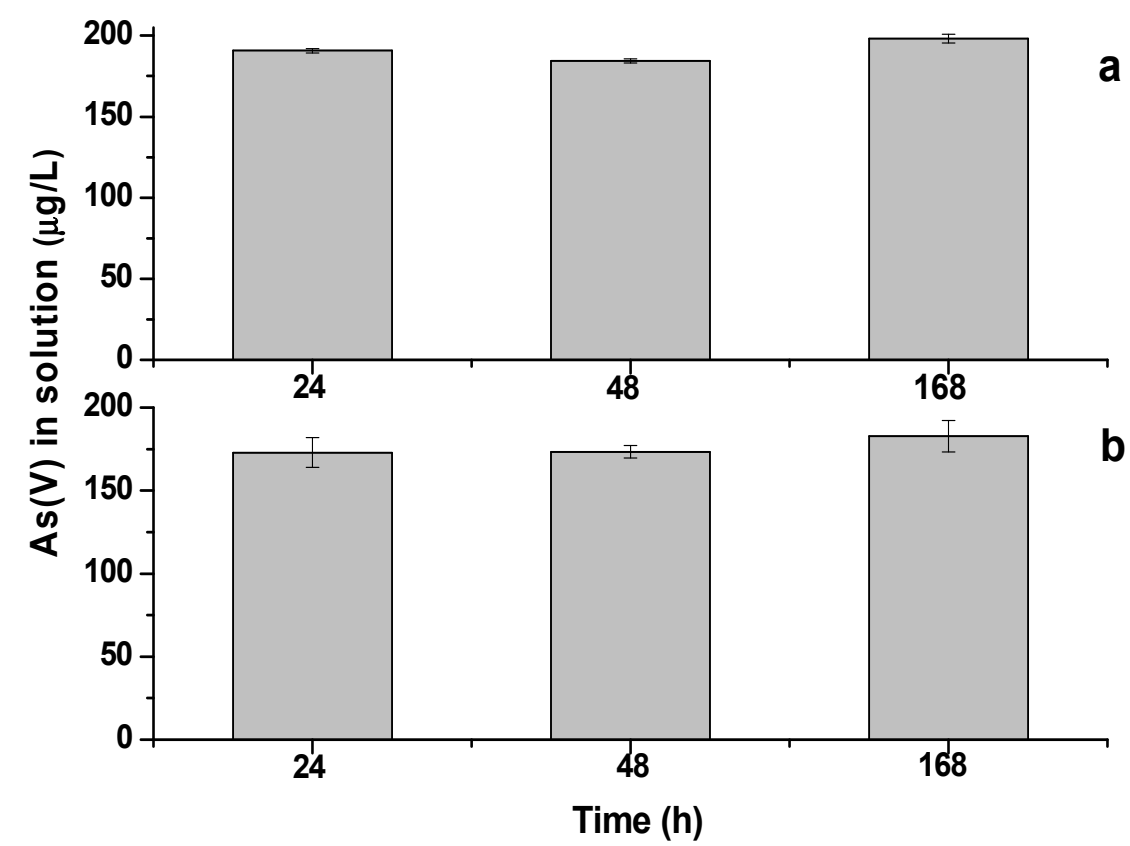

Figure 4. $11 \mathrm{As}(\mathrm{V})(200 \mu \mathrm{g} / \mathrm{L})$ speciation studies in the presence of hematite aggregates in (a) light (b) dark. The columns represent arsenic in solution $(\mu \mathrm{g} / \mathrm{L})$ 


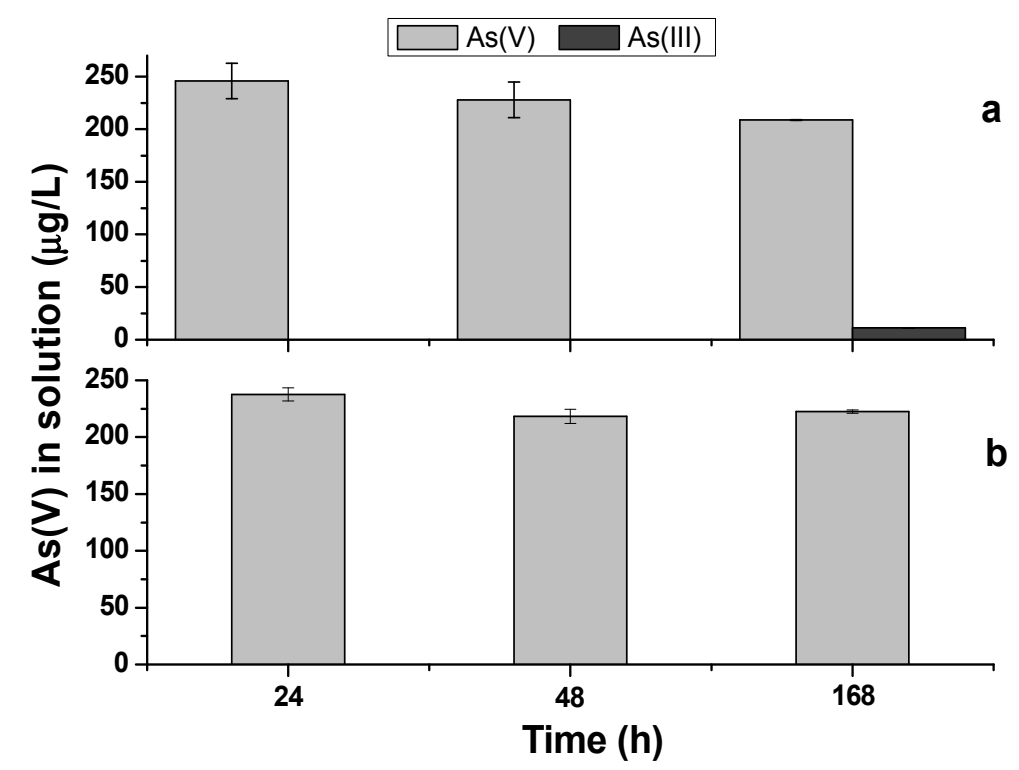

Figure 4. $12 \mathrm{As}(\mathrm{V})$ control $(250 \mu \mathrm{g} / \mathrm{L})$ performed during the As(V)-hematite nanoparticles speciation experiment (a) light (b) dark.

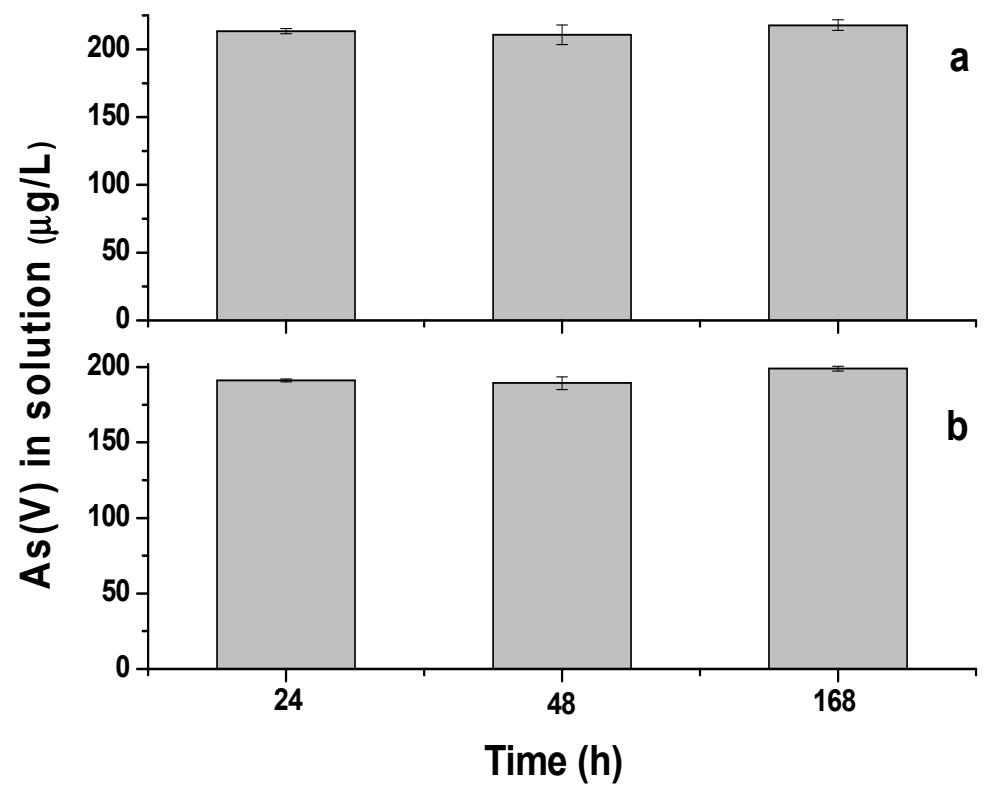

Figure 4. $13 \mathrm{As}(\mathrm{V})$ control $(200 \mu \mathrm{g} / \mathrm{L})$ performed during the As(V)-hematite aggregates speciation experiment (a) light (b) dark 
This can possibly explain why no $\mathrm{As}(\mathrm{V})$ reduction occurred during the photochemical speciation experiments thereby suggesting that other factors are causing $\mathrm{As}(\mathrm{V})$ reduction in the presence of hematite particles. The results here are different from Figure 4.2 where As(V) reduction was observed. The microbial content was not controlled in these experiments and can vary by sample, therefore, the difference in $\mathrm{As}(\mathrm{V})$ reduction might be explained by microbial activity. Based on the experimental conditions, microbial activity could be another possible factor responsible for $\mathrm{As}(\mathrm{V})$ reduction in the presence of hematite nanoparticles and hematite aggregates.

It is well established that $\mathrm{As}(\mathrm{V})$ reduction mainly occurs microbially in the environment (Ahmann et al., 1997; Nicholas et al., 2003; Lloyd et al., 2006). Therefore, the samples in Figure 4.2 might have a higher microbial content than the samples from the photochemical speciation experiments thereby more $\mathrm{As}(\mathrm{V})$ underwent microbial reduction. In order to determine if microbes are causing $\mathrm{As}(\mathrm{V})$ reduction, preliminary studies were conducted to investigate the microbial effect on arsenic speciation. Sodium azide which can minimize microbial oxidation/reduction of arsenic, was added to hematite nanoparticle suspensions to remove microbial effect (Liu et al., 2010). However, sodium azide is a salt and nanoparticles could aggregate rapidly in solutions with high salinity (see Chapter 2). Therefore, stability test on the hematite nanoparticles suspensions were performed. The addition of sodium azide to the hematite nanoparticle suspension and monitoring how fast the particles sediment, provide a good indication on how fast aggregation and sedimentation of the particles occur. Fig 4.14 showed the sedimentation of hematite nanoparticles in the presence of $0.02 \%$ sodium azide. Hematite nanoparticles aggregated rapidly and majority of the particles sediment within $48 \mathrm{~h}$ (Fig. 
4.14). From the speciation experiments, most arsenic species conversion occurred after 24-48h. Therefore, it will be challenging to study the redox transformation of arsenic in the presence of hematite nanoparticles in a microbe free environment when only few particles remained dispersed in the suspension after 48h. Therefore, future experiments are necessary to thoroughly investigate the microbial redox transformation of arsenic in the presence of hematite particles (see Chapter 5). It is also necessary to determine how particle size and stability of the nanoparticle suspension influences the microbial redox transformation of arsenic.

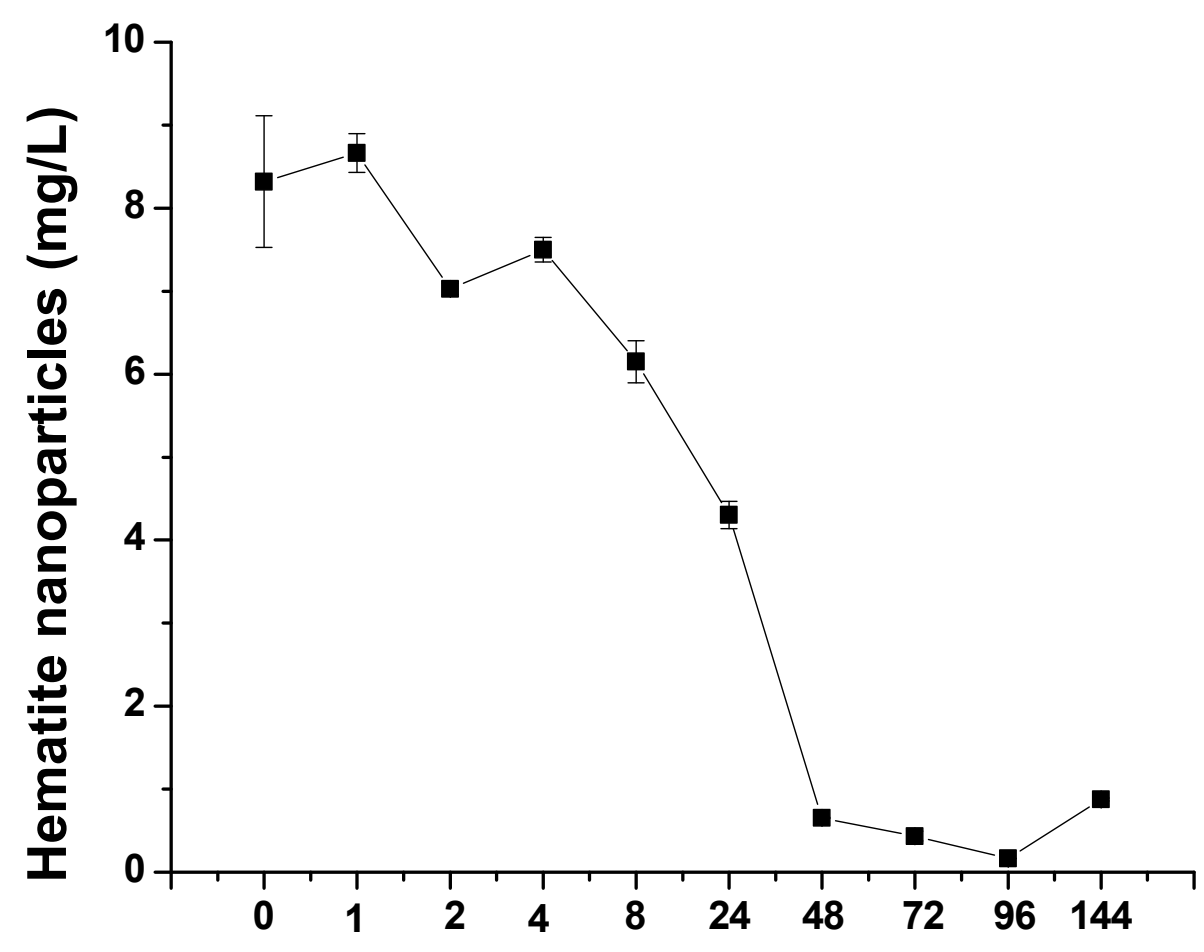

Figure 4. 14 Sedimentation of hematite

Time (h) presence of $0.02 \%$ sodium azide over a period of $144 \mathrm{~h}$. 


\subsection{Conclusions}

This study evaluated the transformation of arsenic species in the presence of hematite nanoparticles and hematite aggregates. The effect of particle size on the rate of species transformation was determined and the possible environmental factors, such as photochemistry, that are responsible for the redox transformation were also investigated. The major findings are that As(III) can be oxidized to As(V) and As(V) can be reduced to As(III) in the presence of hematite nanoparticles and hematite aggregates. In addition, higher amounts of $\mathrm{As}(\mathrm{III})$ and $\mathrm{As}(\mathrm{V})$ was transformed in the presence of hematite nanoparticles than hematite aggregates suggesting that $\mathrm{As}(\mathrm{III})$ and $\mathrm{As}(\mathrm{V})$ readily undergoes redox transformation in the presence of hematite nanoparticles than hematite aggregates. The hematite particle size affects the rate of species conversion. The large surface area of the nanoparticle can accommodate higher amounts of arsenic that can undergo redox transformation faster and in higher amounts on the nanoparticle's surface than on the hematite aggregates' surfaces. Photochemical reactions could play an important role in the redox transformation of arsenic in the presence of hematite particle. Arsenite oxidation in the presence of both hematite nanoparticles and hematite aggregates occurred faster in the presence of light than in the dark. However, minimal As(V) was reduced photochemically. At present, the mechanism of photoinduced oxidation of As(III) is uncertain however on the basis of our results it is proposed that after light absorption, a strong reducing electron and a strong oxidizing hole are formed causing $\mathrm{Fe}(\mathrm{III})$ to be reduced to $\mathrm{Fe}(\mathrm{II})$ by the reducing electron and $\mathrm{As}(\mathrm{III})$ to be oxidized to $\mathrm{As}(\mathrm{V})$ via the oxidizing hole, $\mathrm{h}^{+}$on the particle surface. Both $\mathrm{Fe}(\mathrm{II})$ and $\mathrm{As}(\mathrm{V})$ is released from the mineral surface and into the solution where they newly converted As(V) was detected. 
Future studies are necessary to confirm the proposed pathway of arsenic photoinduced redox transformation. Overall, these results have environmental implications because the redox transformation of arsenic on the mineral surface leads to the release of arsenic into solution making it more bioavailable.

\subsection{List of References}

Ahmann D, Krumholz LR, Hemond HF, Lovley DR, Morel FMM. Microbial Mobilization of Arsenic from Sediments of the Aberjona Watershed. Environ Sci Technol 1997; 31: 2923-2930.

Altman IS, Lee D, Chung JD, Song J, Choi M. Light absorption of silica nanoparticles. Phys Rev B 2001; 63: 161402.

Baumanis C, Bloh JZ, Dillert R, Bahnemann DW. Hematite Photocatalysis: Dechlorination of 2,6-Dichloroindophenol and Oxidation of Water. J Phys Chem C 2011; 115: 25442-25450.

Bhandari N, Reeder RJ, Strongin DR. Photoinduced Oxidation of Arsenite to Arsenate on Ferrihydrite. Environ Sci Technol 2011; 45: 2783-2789.

Bhandari N, Reeder RJ, Strongin DR. Photoinduced Oxidation of Arsenite to Arsenate in the Presence of Goethite. Environ Sci Technol 2012; 46: 8044-8051.

Cummings DE, Caccavo F, Fendorf S, Rosenzweig RF. Arsenic Mobilization by the Dissimilatory Fe(III)-Reducing Bacterium Shewanella alga BrY. Environ Sci Technol 1999; 33: 723-729.

Dickson D, Liu G, Li C, Tachiev G, Cai Y. Dispersion and stability of bare hematite nanoparticles: Effect of dispersion tools, nanoparticle concentration, humic acid and ionic strength. Sci Total Environ 2012; 419: 170-177.

Emett MT, Khoe GH. Photochemical oxidation of arsenic by oxygen and iron in acidic solutions. Water Res 2001; 35: 649-656.

Jain A, Raven KP, Loeppert RH. Arsenite and Arsenate Adsorption on Ferrihydrite: Surface Charge Reduction and Net OH- Release Stoichiometry. Environ Sci Technol 1999; 33: 1179-1184.

Johnston SG, Keene AF, Burton ED, Bush RT, Sullivan LA. Iron and arsenic cycling in intertidal surface sediments during wetland remediation. Sci Total Environ 2011; 45: 2179-2185. 
Langner HW, Inskeep WP. Microbial reduction of arsenate in the presence of ferrihydrite. Environ Sci Technol 2000; 34: 3131-3136.

Liu G, Cai Y. Complexation of arsenite with dissolved organic matter: Conditional distribution coefficients and apparent stability constants. Chemosphere 2010; 81: 890-896.

Lloyd JR, Oremland RS. Microbial transformations of arsenic in the environment: from soda lakes to aquifers. Elements 2006; 2: 85-90.

Luther GW, Rickard DT. Metal sulfide cluster complexes and their biogeochemical importance in the environment. J Nanopart Res 2005; 7: 389-407.

Masscheleyn PH, Delaune RD, Patrick WH. Effect of redox potential and $\mathrm{pH}$ on arsenic speciation and solubility in a contaminated soil. Environ Sci Technol 1991; 25: 1414-1419.

Motamedi S, Cai Y, O'Shea KE. Reactions of Ultrasonically Generated Hydroxyl Radicals with Arsenic in Aqueous Environments. ACS Sym Ser 2003; 835: 84.

Nicholas D, Ramamoorthy S, Palace V, Spring S, Moore J, Rosenzweig RF. Biogeochemical transformations of arsenic in circumneutral freshwater sediments. Biodegradation 2003; 14: 123-137.

Raven KP, Jain A, Loeppert RH. Arsenite and Arsenate Adsorption on Ferrihydrite: Kinetics, Equilibrium, and Adsorption Envelopes. Environ Sci Technol 1998; 32: 344-349.

Shipley HJ, Yean S, Kan AT, Tomson MB. Adsorption of arsenic to magnetite nanoparticles: effect of particle concentration, $\mathrm{pH}$, ionic strength, and temperature. Environ Toxicol Chem 2009; 28: 509-515.

Sosa IO, Noguez C, Barrera RnG. Optical Properties of Metal Nanoparticles with Arbitrary Shapes. J Phys Chem B 2003; 107: 6269-6275.

Stumm W, Morgan JJ. Aquatic chemistry: chemical equilibria and rates in natural waters, 1996.

Tang J, Zou Z, Ye J. Photocatalytic decomposition of organic contaminants by Bi2WO6 under visible light irradiation. Catal Lett 2004; 92: 53-56.

Waychunas GA, Kim CS, Banfield JF. Nanoparticulate iron oxide minerals in soils and sediments: unique properties and contaminant scavenging mechanisms. J Nanopart Res 2005; 7: 409-433. 
Yan B, Wrenn BA, Basak S, Biswas P, Giammar DE. Microbial Reduction of Fe(III) in Hematite Nanoparticles by Geobacter sulfurreducens. Environ Sci Technol 2008; 42: 6526-6531.

Zaspalis V, Pagana A, Sklari S. Arsenic removal from contaminated water by iron oxide sorbents and porous ceramic membranes. Desalination 2007; 217: 167-180.

Zhao Z, Jia Y, Xu L, Zhao S. Adsorption and heterogeneous oxidation of As (III) on ferrihydrite. Water Res 2011; 45: 6496-6504.

Zou Z, Ye J, Sayama K, Arakawa H. Direct splitting of water under visible light irradiation with an oxide semiconductor photocatalyst. Nature 2001; 414: 625-627. 


\section{CHAPTER V}

Summary and Future Research Directions 


\subsection{Summary}

This research furthers the understanding of the effects of iron oxide nanoparticles (hematite nanoparticles) on the fate and transformation of arsenic in the environment. The first step was to understand the environmental behavior of nanoparticles. It is important to understand how IONPs exist in aquatic environments in regards to their particle size, aggregation and stability in the presence of environmental factors such as organic matter and ionic strength. The preparation of a bare commercial IONPs stable dispersion that is within the nanoscale range is an important component for studying the interaction of IONPs with contaminants (e.g., arsenic). A variety of dispersion techniques (vortex, bath sonication and probe ultrasonication) were investigated to disperse bare IONPs. The effects of important environmental factors such as dissolved organic matter and ionic strength on the stability of IONPs dispersions were also investigated. Among all the techniques studied, probe ultrasonication was more effective at dispersing IONPs. At least $50 \%$ or more of IONPs had hydrodynamic diameters ranging from 120-140 nm that changed minimally in size over a period of $168 \mathrm{~h}$. The sedimentation of IONPs was also minimal for a prolonged period of time suggesting that IONPs formed a stable suspension with particles within the nano-range. Over the course of 168 hours, considerable amounts of IONPs remained dispersed in the presence of low ionic strength $(0.1 \mathrm{mM}$ of $\mathrm{NaCl})$ and $100 \mathrm{mg} / \mathrm{L}$ of humic acid (HA) (dissolved organic matter). These results indicated that IONPs can be broken down efficiently into "nanosize range" by probe ultrasonication and a degree of stability can be achieved without the use of synthetic modifiers to enhance colloidal stability. The probe ultrasonication dispersion 
tool could be used to develop a laboratory method to study the adsorption mechanism between dispersed bare IONPs and toxic contaminants.

The adsorption of arsenic onto iron oxide surfaces can affect its mobility and bioavailability. In the environment arsenic is known to adsorb onto bulk zerovalent iron (ZVI), iron oxides and oxy-hydroxides. However, the adsorption process can be enhanced using nanoparticulate iron oxides. The adsorption kinetics and adsorption isotherm studies of arsenic on bare hematite nanoparticles and hematite aggregates were investigated. Using the probe ultrasonication dispersion method to prepare IONPs suspensions, the adsorption of arsenic to hematite nanoparticles were investigated. The adsorption onto hematite aggregates was also investigated using vortex as the dispersion tool to prepare iron oxide in the aggregated form. As(V) and As(III) had similar rate constants as rapid adsorption occurred within the first $8 \mathrm{~h}$ regardless of particle size. However, hematite nanoparticles and aggregates showed a higher affinity to adsorb larger amounts of $\mathrm{As}(\mathrm{V})$ than $\mathrm{As}(\mathrm{III})$ at equilibrium. Adsorption isotherm studies showed that hematite nanoparticles has a significantly higher adsorption capacity for both As(V) and As(III) than hematite aggregates. The large surface area of the nanoparticles can accommodate higher amounts of arsenic for adsorption on their surface. The adsorption and isotherm studies indicated that the size of the iron oxide mineral is a major factor affecting its reactivity as hematite nanoparticles can efficiently adsorb more As(V) and As(III) compared to hematite aggregates. This information can assist in predicting arsenic adsorption behavior and assess the role of iron oxide nanoparticles in the biogeochemical cycling of arsenic. 
The redox transformation process is an important aspect in the biogeochemical cycling of arsenic. The toxicity, mobility and bioavailability of arsenic are highly dependent upon its redox state. Environmental factors such as mineral phases could play a significant role in arsenic redox transformation process. Both iron and arsenic can undergo microbial and photochemcial redox transformation and these processes can be intertwined in the environment. Arsenic speciation studies in the presence of hematite nanoparticles and hematite aggregates were performed. As(III) oxidation to $\mathrm{As}(\mathrm{V})$ and $\mathrm{As}(\mathrm{V})$ reduction to $\mathrm{As}(\mathrm{III})$ occurred in the presence of hematite nanoparticles and hematite aggregates. The particle size is an important factor in the rate of redox transformation as arsenic speciation occurred more readily and rapidly in the presence of hematite nanoparticles than when interacting with hematite aggregates. The hematite nanoparticles have high surface area that can accommodate higher amounts of arsenic for adsorption. Any surface mediated arsenic transformation will occur faster and in higher amounts on the nanoparticle's surface than on the hematite aggregates' surfaces.

Photochemical processes are one of the factors influencing arsenic speciation. Arsenite oxidation in the presence of both hematite nanoparticles and hematite aggregates occurred faster in the presence of light than in dark. However, minimal As(V) was reduced photochemically. Throughout all the speciation studies, the newly converted arsenic species, whether it's As(III) or As(V), were found in the aqueous phase. The mechanism of the photochemical oxidation of arsenic is not well established and on the basis of the results it is proposed that after As(III) adsorption to the hematite surface and upon exposure to light, the insoluble Fe(III) gets reduced to the soluble $\mathrm{Fe}(\mathrm{II})$ and the adsorbed $\mathrm{As}(\mathrm{III})$ is oxidized to $\mathrm{As}(\mathrm{V})$. Both $\mathrm{Fe}(\mathrm{II})$ and $\mathrm{As}(\mathrm{V})$ are then released into the 
aqueous phase. Therefore, the presence of nanoparticles in the aqueous environment can enhance arsenic speciation. The higher amounts of arsenic that is absorbed onto the nanoparticles' surface can be exposed to surface mediated redox reactions therefore resulting in higher amounts of arsenic being reduced or oxidized.

Overall, this research has significant environmental implications. Iron oxide nanoparticles can remain stable in the environment to a certain extent where it can adsorb As(III, V) and influence arsenic speciation. The can affect the mobility and bioavailability of arsenic in the environment. The adsorption of arsenic to the iron oxide surface can reduce arsenic mobility and bioavailability. However, arsenic species conversion on the nanoparticle surface and ultimate release back into the aqueous environment will result in arsenic's increased mobility and bioavailability. The nanoparticles size and aggregation in the natural environment will affect how fast adsorption and redox transformation of arsenic occur. In conclusion, the biogeochemical cycling of arsenic is a complex process and this research provided a better understanding of the role of iron oxide nanoparticles in arsenic cycling in the environment.

\subsection{Future Research Directions}

\subsubsection{Photoinduced arsenic species transformation}

The proposed pathway for the photochemical oxidation of As(III) in the presence of hematite particles needs to be investigated extensively. In order to determine if Fe(III) undergo reduction, the concentration of $\mathrm{Fe}(\mathrm{II})$ and $\mathrm{Fe}(\mathrm{III})$ should be quantified at different time intervals (24, 48 and 168h). Arsenic species concentration should also be measured and compared to the concentration of $\mathrm{Fe}(\mathrm{II})$ during that time period. The presence of $\mathrm{Fe}(\mathrm{II})$ will suggest that $\mathrm{Fe}(\mathrm{III})$ undergoes reduction. In addition experiments 
should be performed to determine whether or not the redox transformation of arsenic is surface mediated or solution based.

\subsubsection{Microbial transformation of arsenic species}

It is necessary to determine the effect of microbes on arsenic redox transformation. Microbes play a significant role in the redox transformation of both arsenic and iron in the environment. The iron redox transformation by microbes is strongly intertwined with arsenic redox transformation in the environment. Dissimilatory microbial reduction of $\mathrm{Fe}(\mathrm{III})$ can cause the release of Fe(II) and any adsorbed $\mathrm{As}(\mathrm{III})$ and $\mathrm{As}(\mathrm{V})$ into solution where the arsenic species can undergo direct oxidation or reduction by the same microbes that caused iron reduction or by other microbes. Therefore, investigating arsenic speciation in a microbe free environment will provide a good indication of how much influence does microbes have on speciation.

The effect of iron oxide particle size on microbial arsenic speciation is important. A few studies in the literature have reported that the rate of microbial Fe(III) reduction can be affected by the size of the iron oxide particle. Nanosized particles are more accessible to microbes than large aggregates thereby undergoing a higher reduction rate. Therefore, it is necessary to the study how the size of the hematite nanoparticle will influence the rate of arsenic speciation in the presence of microbes. The addition of microbes such as geobacter metallireducens and shewanella putrefaciens (common iron reducing microbes) can be added to arsenic-hematite nanoparticle and arsenic-hematite aggregates suspensions to investigate the rate of the microbial reduction of iron and the subsequent oxidation or reduction arsenic. In these experiments, photochemical effect 
should be eliminated in order to investigate the role of microbes only on arsenic redox transformation.

\subsubsection{Effects of environmental factors on the interactions between arsenic} species and IONP

At present, this research has determined the interactions of IONPs and arsenic in water only without any other factors added. In the environment, other factors are naturally present such as dissolved organic matter that can play key roles in the cycling of arsenic. Dissolved organic matter (DOM) is ubiquitous and has importance in metal cycling and possibly has a stabilizing effect on nanoparticles. A multiphase system is necessary to fully understand the interactions of IONPs with arsenic in natural environments. Future studies should focus on adsorption and isotherm studies of arsenic to IONPs in the presence of DOM. Speciation studies is also necessary as to determine how degradation and oxidation of DOM can be coupled to the redox transformation of arsenic in the presence of IONPs. 
VITA

\section{DIONNE C. DICKSON}

\section{EDUCATION}

2007

B.S., Chemistry

Florida International University

Miami, Florida

2013

Doctor of Philosophy, Chemistry

Florida International University

Miami, Florida

PROFESSIONAL EXPERIENCE

2010-2012

Graduate Assistant

Florida International University

Miami, Florida

2007-2010

Teaching Assistant

Florida International University

Miami, Florida

2006-2007

Teaching Adjunct

Florida International University

Miami, Florida

FELLOWSHIPS

2012-2013

Dissertation Year Fellow

Florida International University

Miami, Florida

\section{PUBLICATIONS AND PRESENTATIONS}

Dionne Dickson, Guangliang Liu, Chenzhong Li, GeorgioTachiev, Yong Cai (2012)

Dispersion and stability of bare hematite nanoparticles: Effect of dispersion tools, nanoparticle concentration, humic acid and ionic strength. Science of the Total Environment, 419: 170-177

Dionne Dickson, Guangliang Liu, Yong Cai. Interactions of arsenite and arsenate with hematite nanoparticles and aggregates: sorption kinetics and isotherm studies. (To be submitted) 
Dionne Dickson, Guangliang Liu, Yong Cai. Redox transformation of arsenic in the presence of hematite nanoparticles and hematite aggregates. (To be submitted)

\section{PRESENTATIONS}

Dionne Dickson, Guangliang Liu and Yong Cai. Interaction of arsenate and arsenite with iron oxide nanoparticles: sorption kinetics and adsorption studies. The 9th International Symposium on Persistent Toxic Substances (ISPTS), Miami, Florida, United States, October 23-27, 2012

Dionne Dickson, Guangliang Liu and Yong Cai. Interaction of arsenate and arsenite with iron oxide nanoparticles: sorption kinetics and adsorption studies. 4th Annual Nanoscience Technology Symposium, Miami, FL, United States, September 30- October 1,2011

Dionne Dickson, Yong Cai, Guangliang Liu. Dispersion method to prepare stable iron oxide nanoparticle suspension. 239th ACS National Meeting, San Francisco, CA, United States, March 21-25, 2010

Revoredo, Leslie; Dickson, Dionne; Exposito, Mayra; Tomonto, Andrew; Evans, Andrew; Gregory, Michael; Oliveros, Christian; Ruiz Calderon, Daniel; Roque, Kyle. "Greening" the separation of anthocyanins from coleus plants: TLC and $\mathrm{K}_{\mathrm{a}}$ determination. 235th ACS National Meeting, New Orleans, LA, United States, April 6-10, 2008

Murillo, Jessica; Alexandre, Raphny; Jeannot, Christian; Dickson, Dionne; Castillo, Ace; Valientes, Lorilee; Sebekos, Konstantinos; Gonzalez, Anabel; Juman, Ameera; Hussain, Afirah; Amicarelli, Mariano; Macadar, Daniel; Lopez, Joel; Revoredo, Leslie; Delgado, Milagros; Exposito, Mayra. Art and culture of science: Unique routes for college recruitment. Chemistry Department, Florida International University, North Miami, FL, USA. 235th ACS National Meeting, New Orleans, LA, United States, April 6-10, 2008

Jeannot, Christian; Alexandre, Raphny; Murillo, Jessica; Dickson, Dionne; Revoredo, Leslie; Sebekos, Konstantinos; Exposito, Mayra; Delgado, Milagros. Undergraduate peer teaching assistants in chemistry and biology labs. Abstracts of Papers, 235th ACS National Meeting, New Orleans, LA, United States, April 6-10, 2008

Machado, Jessalyn; Murillo, Jessica; Castello, Angela; Salazar, Nicole; Dickson, Dionne; Amicarelli, Mariano; Espino, Karla; Alexandre, Raphny; Santiesteban, George; Exposito, Mayra; Delgado, Milagros. Driving force of our science club: Attending ACS national meetings. 233rd ACS National Meeting, Chicago, IL, United States, March 25-29, 2007

Dickson, Dionne; Murillo, Jessica; Salazar, Nicole; Exposito, Mayra. Chemistry

Department, Integrating lab techniques and Odyssey software using essential oils. 233rd ACS National Meeting, Chicago, IL, United States, March 25-29, 2007 